\title{
Factors influencing the recruitment, growth and reproduction of a temperate reef fish, Forsterygion lapillum
}

\author{
Paul J. Mensink
}

A thesis submitted to Victoria University of Wellington in fulfillment of the requirements for the degree of Doctor of Philosophy in Marine Biology

Victoria University of Wellington

Te Whare Wānanga o te Ūpoko o te Ika a Māui 2014 
This thesis was conducted under the supervision of:

Dr. Jeffrey S. Shima (primary supervisor)

Victoria University of Wellington

Wellington, New Zealand

Dr. Russell Cole (secondary supervisor) NIWA

Wellington, New Zealand 


\section{Abstract}

In order to gain a comprehensive understanding of population dynamics, it is vital to identify the key factors that contribute to variation in both survival and reproductive success throughout the life history of an organism. The population dynamics of reef fishes may be influenced by events occurring across multiple life stages, throughout the entire life cycle. For instance, the input of new individuals into a population (recruitment) is heavily shaped by mortality in the larval and juvenile stages, which is influenced by conspecific interactions and habitat characteristics. For individuals that survive, variation in somatic growth histories during development may influence mating success among fish that successfully recruit into the adult population, and particular developmental histories may receive a disproportionate amount of a population's reproductive output. However, female preferences for particular phenotypes may also be modified by events occurring in adult life, such as parasite infection. Finally, absolute reproductive success (i.e., the number of offspring that survive to reproductive age) may be dependent upon early larval mortality of offspring, and variation in larval mortality among spawning sites could have consequences for metapopulation dynamics. In this thesis, I investigated how recruitment, growth and reproductive success varied among individuals of a small temperate reef fish, Forsterygion lapillum, the common triplefin, based upon their developmental histories, morphological traits, and habitat characteristics (including conspecific densities, regional locations, etc.). Specifically, I examined:

- how the spatial distribution and survival of juveniles is influenced by age-class interactions (Chapter 2)

- verified methods to measure somatic growth rates during development using scale structures (Chapter 3)

- $\quad$ explored how previous growth rates influence reproductive success (Chapter 4)

- evaluated how reproductive success is modified by the presence of ectoparasites (Chapter 5)

- and finally, assessed how natal origin modifies larval survival probabilities among offspring (Chapter 6). 
The larvae of many reef fishes settle into habitats that are already occupied by adults, and interactions between age classes (intercohort interactions) may affect spatial variation in recruitment strength across settlement sites. In Chapter 2, I evaluated spatial covariation in juvenile and adult densities of $F$. lapillum (within the preferred settlement habitats of juveniles) to investigate correlations between adult and juvenile densities potentially caused by age-class interactions. The relationship between juvenile and adult densities followed a dome-shaped curve, with a negative correlation between juveniles and adults at higher adult densities. The shape of this curve was temporally variable, but was otherwise unaffected by particular features of the site (algal species identity). Using a laboratory-based experiment that used a "multiple predator effects" (MPE) design, I tested the hypothesis that increased settler mortality, caused by either (i) intercohort competition leading to enhanced predation risk or (ii) cannibalism by adults on juveniles, contributed to the observed negative relationship between juvenile and adult densities. Results suggested overall mortality attributable to cannibalism was low; however, smaller settlers appeared to be more vulnerable to cannibalism. There was no evidence that combined or interacting effects between predators ( $F$. lapillum adults and Forsterygion varium [the variable triplefin]) increased predation risk in settlers of F. lapillum. Overall, these results highlight the potentially complex effects adult residents may have on shaping patterns of recruitment and the distributions of new juveniles.

Somatic growth rates through ontogeny are one of the most important metrics for understanding fish populations and in Chapter 3, I evaluate the use of spacing between growth increments on fish scales (called circuli) as a measurement technique for assessing historical growth in F. lapillum. First, I established the relationship between scale growth and body size, and determined how variable this relationship was among populations. The body-scale size relationship was strongly positive and was unaffected by gender; however, there did appear to be significant differences between certain populations. Second, I monitored somatic and fish scale growth in the laboratory to measure the relationship between somatic growth and spacing between growth increments (intercirculus spacing). New scale growth and circuli deposition were both positively correlated with somatic growth. Average intercirculus spacing was also positively correlated with somatic growth rate, but this appeared to differ between age/size classes, with the older and larger individuals showing a weaker relationship. Results suggest that 
intercirculus spacing can be used to determine previous growth histories, but may be limited to particular size/age ranges (e.g., juveniles).

In Chapter 4, I employ the techniques developed in Chapter 3 to examine how early growth rates (derived from fish scales) and male morphological traits explain variation in reproductive output between individual males in F. lapillum. I measured the reproductive success of breeding males in relation to their size and growth rates over the breeding season at two different spawning locations. Clutch size (number of eggs per nest) was highly variable among individuals over the study period; however, I detected a significant, albeit subtle, negative correlation between clutch size and growth rates after settlement. Although growth explained relatively small amounts of total variation, it was the only male trait I measured that significantly correlated with clutch size. The negative effects of faster growth on clutch size were greatest during the period of growth after settlement suggesting that growth at this early stage may be important for later reproductive success (early post-settlement).

In Chapter 5, I examined how infection with an ectoparasite modified reproductive success among individual males using a field survey. Females often preferentially mate with unparasitised males, and therefore parasitised males experience lowered reproductive success. In this study, individuals of greater total length were more likely to be infected with an ectoparasite, but were also more likely to have an egg nest. Parasite infection had no effect on reproductive success (either the presence of a nest, or the average surface area of eggs if a nest was present). Positive covariation in total length, reproductive success, and parasite infection potentially suggest that the influence of parasitic infection on reproductive success may depend upon the strength of selection for larger male body size. In addition, this study provides the first quantitative measurement of ectoparasite infection for both the focal parasite species (Caligus buechlerae) and the host (F. lapillum).

Finally, in Chapter 6, I explore how larval survival is mediated by spawning location. In marine reef fish, spatially isolated adult populations may be connected (i.e., have gene flow) via larval dispersal; however, differential larval survival between source populations may mediate both the degree of population connectivity as well as the reproductive success of individuals within those source populations. To evaluate variation in larval quality among different spawning locations, I conducted a laboratory assay to measure the potential effects of source 
population on larval time to starvation, as starvation is often proposed as a major source of mortality for larval fish. Average survival time was 3.75 days, but survival analysis indicated that starvation resistance did not differ between the two natal sources. For individual nests, mean larval size was negatively correlated with their mean survival time, although this was only apparent in larvae collected from one population (the south coast). My findings indicate that variation in larval traits between source populations does exist, but that on average, source populations had equal resistance to starvation. Given the differences between source populations in (i) the relationship between larval mortality and larval size (i.e., the absence of size effects in one source population) and (ii) overall variation in larval size (larger larvae on the south coast), the relative contribution of larvae from each source population may vary under certain conditions (e.g., low levels of food availability).

In conclusion, the field surveys and laboratory experiments conducted in this thesis demonstrate the potential for a variety of factors across multiple life history stages to influence recruitment, growth and reproduction. These findings suggest that factors across multiple life stages (e.g., conspecific density, previous growth histories, or spawning site) have the ability to influence individual success, and in turn populations. By carefully considering and integrating these factors into our studies of population dynamics, we may be able to gain a more comprehensive understanding of the spatio-temporal fluctuations in populations for marine reef fish. 


\section{Publications from this Thesis}

\section{Published articles:}

Mensink PJ and Shima JS (2014) Patterns of co-occurrence and interactions between age classes of the common triplefin, Forsterygion lapillum. Marine Biology 161:1285-1298. doi: 10.1007/s00227-014-2418-6

Author contribution: PJM performed data collection, analyzed the data and wrote the manuscript. JSS edited the manuscript.

\section{Articles in review:}

Mensink PJ, Geange SW, and Shima JS (in review) Reproductive success of parasitized males in a marine reef fish. Marine Biology

Author contribution: PJM designed the study, performed data collection, analyzed the data and wrote the manuscript. SWG and JSS edited the manuscript. 


\section{Acknowledgements}

First and foremost, I would like to thank my primary supervisor, Jeff Shima, for his support, understanding and encouragement throughout my $\mathrm{PhD}$. Thanks for being patient and letting me develop. I would like to say a big thank you to my secondary supervisor, Russell Cole, who was an excellent source of advice. You were a very kind and generous man, and one of my fondest memories in New Zealand was the lovely dinner I spent with your family. I hope that this thesis can serve your memory proud.

Assistance provided by Anna Smith, Ale Perez-Matus, and Phillip Neubauer at the outset of this thesis was greatly appreciated. I gratefully acknowledge the help I received from an incredible list of volunteers including Alison Smeaton, Eloise Ashworth, Natalie Reiner, Lauren Watwood, Olivia Eaton, Henrietta Woo, Talia Dowd, David Garrett, Cassandre Guinut, Jenny Oliver, Dana Morton, Danelle Lekan, Don Nelson, John Van der Sman, Abi Powell, Alix LaFerriere, Steve Jenkins, Daniel McNaughtan, Cesar Cardenas and Shane Geange.

A special thanks to Abi for being my calm voice of reason, Alix for keeping the train on the tracks as it was pulling into the station and Phoebe, for clearing a path through my other responsibilities! I would also like to thank the Chilean Food Scholarship (Anita!) and Cesar Cardenas (my devoted running mate). To glory! Editing assistance provided by Suse Mauer was very much appreciated. I would like to thank the VUCEL Technical support team (John Van der Sman and Daniel McNaughtan) for assistance with the research conducted in this thesis. To 396 The Esplanade, you were my second home, and occasionally my only home, thanks for putting a roof over my head. In addition, I would also like to thank four anonymous reviewers who made extremely helpful comments on Chapters 2 and 5. Furthermore, this thesis has been greatly improved by comments and suggestions made by my three examiners.

Ma Cassu, merci beaucoup pour ta patience, tu as souffert le pire de ce monstre à sept têtes, mais ma chérie je l'ai vaincu! Désormais nous pouvons nager ensemble, ton poisson est arrivé!

Thanks to my overwhelmingly supportive and understanding group of friends, who let me relax when I needed to, your support during this period was unbelievable (especially through the last part of this journey). I would like to express my very great appreciation to my dear friends, Julie Deslippe and Hinrich Schaefer, for their compassionate encouragement for the past 4 years. A very special thank you to my flatmates, Ryan and Dave. for hanging tough, being so understanding and convincing me to take a break when I needed one.

Thanks to everyone, my friends and family alike, who stood by me willingly, while I insisted on putting my work first. You've lived on the margins for the past 4 years, but I promise you will never have to be there again. Thanks for waiting. 
I must pay special mention to three people in particular, without whom, I cannot even begin to imagine how I would have completed this thesis.

Snout, I literally would not have been able to come here without you! And in the end, your advice and guidance was absolutely invaluable. Thanks for your perseverance, your patience, and for always going far out of your way to help me. You revolutionized the way I think about diving and the way I conduct myself as a diver. Thanks especially for the uncountable number of great post-erg/run early morning breakfast meetings. They were an awesome way to start my long days. And of course, thanks for that absolutely delicious ginger crunch, man I'm going to miss that!

I owe a great debt of gratitude to Tim Jones for his insightful and constant advice. Quite simply, you have completely changed the way I think about science. You did the equivalent of teaching a man how to fish; although, I must admit you occasionally gave me a trawler instead of a fishing rod, but you were always there to take the wheel if the ship got into rough seas. Your influence is splashed throughout the pages of this thesis and I only hope I have done well by your efforts. I know you'll be an inspiration for years to come because I'll always be trying to catch up with you! I will always look back very fondly on our chats, which spanned the entire universe of topics and served as well timed breaks from a long workday.

Finally, a huge thank you to Shane Geange. I could not have completed this thesis without you. You and your work serve as a constant source of inspiration for me. As good as my third supervisor, you showed me how to be a good biologist and your lessons will forever shape the way I conduct myself as a scientist. Shane, you watched me stumble and fall down, but always waited patiently while I picked myself back up (even when you had warned me about it!). My dear friend, I will never forget what you have done for me.

I would like to say a massive thank you to my brother Dave and my entire family, who have always supported me throughout my entire academic career. Lastly, I would like to say a very special thank you to my parents, Cheryl and Fred, who have given me everything. At the beginning of this journey I wrote to you with a quote, that I felt perfectly encapsulated my feelings at the time, I wish to echo this same sentiment again:

"And now has begun what I have always thought to be the supreme joy in life. And I am loving it. Today was so rich that now, in the evening, I must collect myself a little, and so I am writing to you to thank you, dear parents, for having given me all this happiness."

Felix Mendelssohn, October 10, 1830

And so life begins anew... 


\section{Table of Contents}

Abstract

Publications from this Thesis $v$

Acknowledgements $v i$

Table of Contents viii

List of Figures $x i$

List of Tables $x v i$

Chapter 1 - General Introduction

Chapter 2 - Patterns of co-occurrence and interactions between age classes of the common triplefin, Forsterygion lapillum

2.1 Abstract

2.2 Introduction

2.3 Methods

2.3.1 Density surveys

2.3.2 Statistical models and data analysis of density surveys

2.3.3 Experimental test of multiple-predator effects and cannibalism

2.3.4 Experimental set-up and trials

2.3.5 Statistical analysis for experimental trials

2.3.6 Statistical software

\subsection{Results}

2.4.1 Density patterns among sites, algal species, and sampling periods

2.4.2 Relationship between juvenile and adult densities

2.4.3 Experimental test of intercohort multiple-predator effects and cannibalism

2.5 Discussion 40

Chapter 3 - Application of intercirculus spacing to measure body growth in the common triplefin

\subsection{Abstract}

\subsection{Introduction} 46

3.3 Methods 47

3.3.1 Validation of length-body scale relationship 47

3.3.2 Scale removal and measurement 48

viii 
3.3.3 Statistical analysis of body- scale length relationship ___ 50

3.3.4 Growth monitoring ___ 51

3.3.5 Statistical analysis of growth monitoring trials __ 52

3.3.6 Statistical Software___ 54

3.4 Results 56

3.4.1 Body-scale relationship ___ 56

3.4.2 Growth monitoring ___ 58

3.5 Discussion ___ 64

Chapter 4 - The effects of previous growth history on male reproductive success in the common triplefin, Forsterygion lapillum___ 67

4.1 Abstract___ 68

4.2 Introduction ___ 69

4.3 Methods__ 71

4.3.1 Field Collections____ 71

4.3.2 Measurement of egg number and egg size ___ 72

4.3.3 Estimating growth history ___ 73

4.3.4 Statistical analysis ___ 77

4.3.5 Statistical software ___ 78

4.4 Results _ 79

4.4.1 Differences in male characteristics between spawning locations ___ 79

4.4.2 Clutch size models ___ 79

4.4.3 Egg size model selection __ 85

4.5 Discussion____ 87

Chapter 5 - Reproductive success of parasitised males in a marine reef fish__ 90

5.1 Abstract____ 91

5.2 Introduction ___

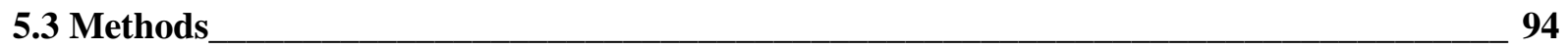

5.3.1 Study species ___ 94

5.3.2 Surveys _ 97

5.3.3 Statistical Analysis ___ 98

5.4 Results _ 99

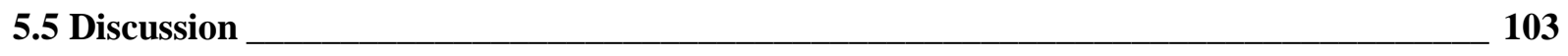

Chapter 6 - The effects of natal source on larval starvation resistance in a temperate reeffish _ 105

6.1 Abstract____ 106 
6.2 Introduction

6.3 Methods

6.3.1 Collection of larvae

6.3.2 Assay design

6.3.3 Statistical Analysis

6.4 Results

6.5 Discussion

Chapter 7 - Contributions and discussion

7.1 Patterns of co-occurrence among adults and juveniles 123

7.2 Measuring ecologically relevant growth using fish scales

7.3 Male reproductive success is influenced by previous growth 124

7.4 Parasitic effects on reproductive success

7.5 Relationships between starvation resistance and larval size may cause source populations to differ in larval mortality 125

7.6 Concluding remarks 126

Appendix A-Description of model averaging 127

Appendix B - Determination of scale size at formation 129

Appendix C - Sensitivity Analysis for Deming regression_ 134

Appendix D - Simple simulation for larval mortality from two source populations 138

Literature Cited 141 


\section{List of Figures}

Figure 1.1 Red box within panel A shows the location of the study site within New Zealand, enlarged in panel B, with the specific study areas for each chapter indicated by a colour-coded number.

Figure 2.1 A The two fish species used in experimental trails: adult Forsterygion varium (predator species) and the adults and settlers ( 10 days past settlement) of Forsterygion lapillum. Scale bar at the bottom of the photograph is in millimetres. B Experimental trial tanks $(0.9 \mathrm{~m} 2$ bottom surface area) with a sandy bottom, two stipes of Cystophora retroflexa and a light covering of cobbles to provide cover. The white stand-pipe used for excurrent flow is shown on the left side of the tank

Figure 2.2 Densities of juvenile (panel A) and adult (panel B) F. lapillum (mean \pm SE) at six sites within two locations around the Miramar peninsula (Harbour: Hazard Bay, Shark Bay, Point Halswell; exposed southern coast: Moa Point, Reef bay Waitaha Cove) over three sampling periods (harbour: 9 Feb, 18 Feb, 9 March; exposed southern coast: 10 Feb, 20 Feb, March 3). Sites with different lowercase letters represent significantly $(\mathrm{p}<0.05)$ different site means based on a post-hoc Tukey's test from a generalized linear mixed model with site as a fixed factor and a random factor of quadrat ID (sampling dates pooled)

Figure 2.3 Densities of juveniles (panel A) and adults (panel B) in quadrats of Carpophyllum maschalocarpum (black bars) and mixed quadrats containing 50\% Carpophyllum maschalocarpum and 50\% Cystophora retroflexa (grey bars) over three sampling periods (harbour: 9 Feb, 18 Feb, 9 March; exposed southern coast: 10 Feb, 20 Feb, March 3). Results are pooled by location. Errors bars represent the mean \pm standard error

Figure 2.4 Predicted juvenile densities (bold line) for the quadratic model, split by sampling period (harbour: 9 Feb, 18 Feb, 9 March; exposed southern coast: 10 Feb, 20 Feb, March 3) and location. Plots are given for visualisation purposes and do not accurately reflect the variation that is accounted for the by the random effect of site in the full statistical model. As there were no significant differences between macroalgal species, predictions shown are based on Carpophyllum habitats only to ease interpretation. Dashed lines around the model fit represent confidence intervals ( $2 \mathrm{x}$ unconditional standard errors) for the predicted model fit. Points represent a single quadrat density $\left(n=36\right.$ panel $\left.^{-1}\right)$, shifted by 0.1 axis increments to facilitate visualisation of overlapping points. Sites are marked by different shape patterns: Harbour Hazard Bay ( $)$, Shark Bay $(\boldsymbol{\otimes})$, Point Halswell ( $\mathbf{\square})$; South coast - Reef Bay (十), Moa Point (A), Waitaha Cove (*). Vertical dashed lines represent $95 \%$ confidence intervals on the peak value of the quadratic curve 
Figure 2.5 A Mean proportion \pm SE of $F$. lapillum settlers consumed over a 24 hour period ( $\mathrm{n}=8$ trials) across the 6 experimental treatments. Letters represent statistically significant groups based on a post hoc test $(\alpha=0.05)$ from a generalized linear model with a binomial family. B Comparisons between mean proportion $\pm \mathrm{SE}$ of $F$. lapillum settlers consumed over a 24 hour period in the multi-species treatment and expected proportion \pm SE of settlers consumed determined from additive and substitutive calculations. There was no significant difference between observed mortality in the multi-species treatment and the expected mortality based on either the substitutive or additive designs, indicating that emergent mortality effects were not observed

Figure 3.1 A An individual $F$. lapillum with the area of scale removal highlighted B A photograph of a removed scale under 50X magnification showing the line of scale size measurement in red.

Figure 3.2 Histogram of total length in fish collected for laboratory monitoring of growth rate. The red line represents the selected cut-off point separating the first and second groups. Bin width is $2 \mathrm{~mm}$.

Figure 3.3 Regression fits between total length and scale length for four sites from the Wellington region. Regression fits are separated by site (indicated by colour).

Figure 3.4 Histogram of regression estimates between total length and scale length for 1000 unique random scale selections (from one of two scales) separated by collection site. Each count represents one of the regression estimates taken from the 1000 data sets.

Figure 3.5 Relationships between somatic growth, scale growth, and total length A The relationship between total amount of somatic growth and the total amount of scale growth ( $\mathrm{n}=$ 43) B The relationship total amount of somatic growth and the number of circuli formed on the new scale growth $(n=43) C$ The relationship between average daily growth rate and the total length of the fish at the beginning of the experiment $(n=46) D$ The relationship between average daily growth rate and the average intercirculus spacing (regression lines done separately for each group, $\mathrm{n}=38$ ). Groups are identified by colour (group 1-, group 2- ๑). Regression lines represent a linear regression between the predictor and the response (except for panel $\mathrm{d}$, where regressions were done separately for each group). Grey confidence bands represent the confidence interval on the linear regression fit. 63

Figure 4.1 A non-regenerated scale from an adult common triplefin, Forsterygion lapillum (anterior side on the left and posterior on the right). In order to determine examine previous 
growth, intercirculus spacing was measured from the 6th circuli to the 16th (red line) and from the 17 th circuli to the 27 th (blue line).

Figure 4.2 The effect of time of spawning on clutch size over the breeding season for Forsterygion lapillum males collected from two different spawning locations (represented by two different coloured lines). Errors bars are $95 \%$ confidence intervals. The third sampling period (November) is significantly different from the two samples taken earlier in the breeding season (locations pooled).

Figure 4.3 Relationship between clutch size (the number of eggs in a nest at sampling) and standardized intercirculus spacing (i.e., individual growth relative to other fish at that site) in $F$. lapillum for the three sampling periods (September, October, and November). To ease visualization, other model covariates were fixed to their means. Standardized growth values (based on average intercirculus spacing over circuli 6-16) were calculated separately for each site (Moa Point and Kau Bay) because growth rates differed between sites. Positive standard growth values indicate that an individual grew faster than average at that site, while a negative value indicates that males grew slower than average at that site. Each point represents a single nest and the corresponding male $(n=93)$. The November sampling period was the only time when standardized average intercirculus growth correlated significantly with the number of eggs in a clutch. Grey area around the mean represents $95 \%$ confidence intervals on the regression line fit.

Figure 5.1A Photographs of parasite infections (Caligus buechlerae) on males of the common triplefin (Forsterygion lapillum) B A male common triplefin with three C. buechlerae. Uppermost parasite is a female with distinctive egg sacs trailing the body

Figure 5.2A The probability of parasite infection based on total length (x-axis) for male adult triplefins surveyed at Moa Point B The probability of males having a nest based on total length (x-axis). To ease visualization, model covariates for the egg model were fixed to their means. Grey areas represent standard error of the model fit including variance from the random effect, but not accounting for uncertainty in estimating the random error variance. Tick marks on the top and bottom axis represent individual fish (infected $=1$, uninfected $=0$ ) and are adjusted by $0.3 \mathrm{x}$ axis increments to indicate point density

Figure 6.1 Kaplein-Meier survival curves showing the survival rate over time between the harbour (red line) and the south coast (blue line) for two collection dates. 95\% confidence intervals are based on fixed effects only (i.e., only variation within locations) and are shaded around the lines for both the harbour (red shading) and the south coast (blue shading). 
Figure 6.2 A Mean egg diameter \pm SE and $\mathbf{B}$ Mean larval size \pm SE. For both egg size and larval size, I took the average value for each nest before calculating the confidence intervals, to avoid the effects of pseudoreplication (e.g. multiple larvae from a single nest) on the calculation. However, $\mathrm{p}$-values are calculated from the linear mixed model, which accounted for the correlations between nests using a random factor of nest. Note that egg measurements and larval sizes were not taken from the same nests, and therefore are not directly comparable (i.e., I am unable to conclude that larger larvae on the south coast originated from larger eggs). 116

Figure 6.3 Deming regressions between mean survival time and mean larval length among nests collected from two different source locations (harbour and south coast). Each point represents one nest and error bars represent standard errors for both mean survival time (vertical) and mean larval length (horizontal). The regression line for the harbour is not significantly different from zero (Deming $\beta=-0.423$, bootstrapped 95\% CI $=[-43.065,69.499]$ ), but it is for the south coast (Deming $\beta=-3.938$, bootstrapped 95\% CI $=[-8.727,-0.999]$ ). In each panel, shapes represent collection sites within source populations: harbour (-Kau Bay, $\mathbf{\Delta}$ - Mahunga Bay); south coast ( $\mathbf{O}$ - Moa Point, $\boldsymbol{\Delta}$ - Wahine Point).

Figure B1 A logistical regression showing the probability of squamation (initial scale formation) based on fish length. Histograms on the upper and lower axes show the frequency of observations.

Figure B2 The relationship between total length and number of circuli for fish that had formed scales $(n=10)$. Full data set shown in panel A model with removed outlier shown in panel B. Grey band represents confidence intervals for the regression fit.

Figure C1 1000 regression estimates for the relationship between mean survival time and mean larval size from the harbour using randomly selected variance ratios for both mean survival time and mean length. Left panel represents how the regression estimates changed with variance in average length and right panel shows how the regression estimates change with variance in survival time (regression estimates for each panel are both from the same pool of 1000). Black rug marks across the bottom axis represent the actual variance measurements from each nest and the red rug mark indicates the mean variance value, which was used in the selected model. _ 136

Figure C2 1000 regression estimates for the relationship between mean survival time and mean larval size from the south coast using randomly selected variance ratios for both mean survival time and mean length. Left panel represents how the regression estimates changed with variance in average length and right panel shows how the regression estimates change with variance in survival time (regression estimates for each panel are both from the same pool of 1000). Black rug marks across the bottom axis represent the actual variance measurements from each nest and the red rug mark indicates the mean variance value, which was used in the selected model. _ 137 
Figure D1 A Simulation of the number of surviving larvae per day since hatching and B Percentage of larvae in the larval pool from each source population. Confidence intervals are plotted as shaded bands around the mean line; however, confidence intervals were extremely small and are only visible for the south coast as a slight thickening of the line (panel A). If food becomes available early on in larval development (Days 1 and 2) then the relative contribution of each natal source to the overall larval pool is very similar. If food becomes available on days 3 and 4 , the harbour contributes slightly more larvae to the pool because the larger larvae on the south coast have begun to die off earlier. If food does not become available until later on days 5 and 6, then the south coast provides the majority of the larvae to the larval pool. This simulation is intended for illustrative purposes only and is not considered an actual predictive model._ 140 


\section{List of Tables}

Table 2.1 Hypothetical relationships between juvenile densities (y axis) and adult densities ( $\mathrm{x}$ axis), and the underlying processes that may be responsible for each relationship (assuming overlap in habitat use between age classes occurs). The type of settlement pattern required for each process is described as (i) selective - settling larvae display habitat preferences and are able to choose settlement habitats, (ii) passive - settling larvae are unable to select specific settlement habitats and settle randomly, or (iii) either- the process can occur regardless of whether or not settlement is selective or passive. In addition, the possibility that a particular process could lead to intercohort density dependence (DD) is indicated by a yes $(\mathrm{Y})$ or a no $(\mathrm{N})$. In some cases (marked with an *), density dependence could only occur if (i) settlement is selective and (ii) there is lower survival in the selected habitat. The authors acknowledge that this is not an exhaustive list of potential hypotheses, but rather a list of observed processes in the literature.. 17

Table 2.2 Experimental treatments for laboratory experiment including additive and substitutive designs along with the corresponding process they aimed to quantify..... 26

Table 2.3 Standardised (input variables scaled to a mean of 0) model-averaged regression estimates for combined model (that included sampling period as fixed factor). Unconditional standard errors (SE) include model selection uncertainly in their estimates. Upper and lower confidence intervals (CI) are given on the regression estimates. Relative importance (RI) gives the sum of the AICc weight for each model that parameter was included in (a RI of 1 indicates that parameter was present in all of the averaged models). For the interaction between adult density and sampling period, individual regression estimates are given for each level of sampling period (e.g., Sample 2, Sample 3), but in all cases the models included interactions between all levels of sampling period. $P$-values are based on Wald test $Z$ scores with significant values given in bold 36

Table 2.4 Standardised (input variables scaled to a mean of 0) model-averaged regression estimates for each sampling point separately. The number of models averaged for each sampling was 2, 4, 3 respectively. Unconditional standard errors (SE) include model selection uncertainly in their estimates. Upper and lower confidence intervals (CI) are given on the regression estimates. Relative importance (RI) gives the sum of the AICc weight for each model that parameter was included in (a RI of 1 indicates that parameter was present in all of the averaged models). For the interaction between adult density and sampling period, individual regression estimates are given for each level of sampling period (e.g., Sample 2, Sample 3), but in all cases the models included interactions between all levels of sampling period. P-values are based on Wald test $\mathrm{Z}$ scores with significant values given in bold 
Table 3.1 Regression outputs ( $\beta$ ) from linear regression between total length and scale length for sites pooled and sites separated including confidence intervals on the regression estimate (CI), $F$ values, degrees of freedom (df), $p$ values, and coefficient of determination (R2).

Repeated analysis shows the mean regression coefficients \pm standard deviation and the mean $\mathrm{R} 2$ values \pm standard deviation taken from 1000 datasets constructed by randomly selecting one of the two prepared scales for each collected fish......................................................................... 57

Table 3.2 Regression coefficients, $t$-values and $p$-values for 4 different models from the laboratory monitoring of somatic growth rates.

Table 4.1 Means \pm SE in male characteristics between Kau Bay and Moa Point for all males pooled across sampling dates. F statistics including degrees of freedom (df) and $p$ values are based on one way ANOVAs between each variable with an independent factor of spawning location. 80

Table 4.2 Standardized (input variables scaled to a mean of 0) model-averaged regression estimates for models with a response variable of clutch size including averaged intericulus spacing between scale circuli 6-16 or circuli 17-27 fit as a fixed factor. Unconditional standard errors (SE) include model selection uncertainly in their estimates. Upper and lower confidence intervals (CI) are given on the regression estimates. Relative importance (RI) gives the sum of the AICc weight for each model that parameter was included in (a RI of 1 indicates that parameter was present in all of the averaged models). For the interaction between sampling period and intercirculus spacing and between sampling period and Fulton's K individual regression estimates are given for each level of sampling period (e.g., Sample 2, Sample 3), but in all cases the models included interactions between all levels of sampling period. P-values are based on Wald test $\mathrm{Z}$ scores with significant values given in bold. Averaged model for circuli 616 included estimates from 6 models and averaged model for circuli 17-27 included estimates from 9 models.

Table 4.3 Standardized (input variables scaled to a mean of 0) model-averaged regression estimates (Sample 1 and Sample 3) or mixed model regression estimates (Sample 2) from models for each sampling period. Intercirculus spacing is for circuli 6-16. Unconditional standard errors (SE) include model selection uncertainly in their estimates. Upper and lower confidence intervals (CI) are given on the regression estimates. Relative importance (RI) gives the sum of the AICc weight for each model that parameter was included in (a RI of 1 indicates that parameter was present in all of the averaged models). P-values for sample 1 and sample 3 are based on Wald test $Z$ scores, while sample 2 is based $t$ values. Significant values are given in bold. 
Table 4.4 Model-averaged standardized regression coefficients, unconditional standard errors, confidence intervals, and $\mathrm{p}$ values (based on Wald $\mathrm{z}$ scores) for fixed effects in the egg size model. Sample one is the intercept and all other estimates are the effects on average egg size based on an increase of one standard deviation in the fixed variable. Unconditional standard errors take into account model uncertainty across several models. Percent change in mean egg size is taken from the average for sampling one (the intercept) for time of spawning and location. Percent change for Fulton's $\mathrm{K}$ is taken from the average of the appropriate sampling period..... 86

Table 5.1 Top 2 models for each of the two binomial mixed models (parasite prevalence and egg presence) and the linear mixed model (egg area) based on AICc values. Table also includes fixed effect estimates ( $\beta, \log$ odds), bootstrapped confidence intervals (CI, bias corrected and accelerated method for binomial models and percentile method for linear model, simulations $=1000$ ), and significance values for each fixed effect (Wald $\mathrm{z}$ scores*). Akaike weights (w) are calculated for the two top models only (i.e. weights sum to 1.00) and give an indication of the strength of support for that particular model. In both cases, the top model was used for inference and is highlighted in gray. For the fixed effect of local infection (\% infected), the regression estimate represents a $1 \%$ increase in infection. $*$ The p-value for the effect of total length on nest size was determined using a likelihood ratio test, not a Wald z score (see Results)

Table B1. Predicted sizes from linear model between total length and circuli number including standard error of the predictions and prediction (tolerance intervals) on the predicted size...... 133 


\section{Chapter 1 - General Introduction}


One of the main aims of ecology is to identify the factors that shape population dynamics of organisms. Benthic (bottom-associated) marine organisms typically have a complex life cycle that consists of a pelagic larval stage followed by a benthic stage (Thorson 1950; Leis 1991) and variation in recruitment (the number of individuals that successfully transition from the larval to the juvenile stage), is considered a key driver of population dynamics (Victor 1983; Jones 1990; Booth and Brosnan 1995; Caley et al. 1996). However, population dynamics may be controlled by a combination of demographic processes that occur over the entire life history of an organism (Roughgarden et al. 1988; Possingham and Roughgarden 1990; Gimenez 2006), and there has been considerably less emphasis on processes occurring after recruitment (e.g., differential growth or reproductive output among survivors) or what consequences these processes have for populations (Podolsky and Moran 2006). Furthermore, in stage-structured populations, later life stages may alter the survival and distribution of younger life stages (e.g. juveniles, Webster 2004; Osman and Whitlatch 2004; Samhouri et al. 2009; Hunt et al. 2011). Consequently, a more complete and holistic understanding of the factors that shape population dynamics may require an integration of the complete set of processes that occur across the entire life history of an organism (e.g., Eckman 1996; Podolsky 2003; Podolsky and Moran 2006). My thesis aims to address this gap, using a New Zealand marine reef fish, Forsterygion lapillum (the common triplefin), as a model species.

Marine reef fish are among the most functionally diverse species groups found in shallow coastal areas (Micheli and Halpern 2005). Reef fish can have strong effects on reefs, which may include substantial modification of benthic community structure (Choat 1982), moderating phase shifts between system states (Hughes 1994; Bellwood et al. 2004), and regulating nutrient cycling (Nelson et al. 2013). Reef fish are similar to many other marine reef organisms in that they have a bipartite life cycle with pelagic and benthic stages. Their reef-associated life begins when pelagic larvae, typically originating from multiple source populations (Cowen 2000; Standish et al. 2008; Planes et al. 2009), arrive at the reef and metamorphose into juveniles. During the transition from the larval phase, mortality rates can be extremely high (> 50\% mortality in the first two days following settlement, Doherty et al. 2004; Almany and Webster 2006) and this mortality is thought to be strongly driven by predation (Hixon and Carr 1997; Webster 2002). During growth and development on the reef, many species show limited movement from their settlement locations (Reese 1973; Chapman and Kramer 2000), but post- 
settlement movement varies among species. Propensity for post-settlement movement may also depend upon size (Frederick 1997), with smaller fish maintaining home ranges of a few meters (Pomacentridae, Sale 1971; Gobiidae, Luckhurst and Luckhurst 1978; Tripterygiidae, Clements 2003) and larger fish often migrating 100s or 1000s of meters (Cheilodactylidae, Lowry and Suthers 1998; Serranidae, Lowe and Topping 2003). Reef fish display a wide range of courtship and reproductive strategies (Warner 1984; Hastings and Peterson 2010), and mating systems appear to be highly variable between temperate and tropical species groups (Johannes 1978). Fish larvae generally hatch from pelagic or demersal (attached to the substrate) eggs (Thresher 1984) and reef fish subsequently develop (and presumably disperse) for weeks or months before settling back onto reefs (Brothers et al. 1983; Bay et al. 2006; Kohn and Clements 2011). The vital processes of recruitment, growth, and reproduction all occur during reef-associated life, and in this thesis I examine how various factors influence these processes and determine success (survival or reproductive output) in three life history stages (larval, juvenile, and adult). In order to do this, I integrate field and laboratory studies to evaluate the influence of stage-specific factors that may strongly shape population dynamics. This first chapter serves to broadly introduce the areas of research and the questions that are to be addressed in each of the subsequent data chapters. Each chapter is written as a stand-alone manuscript to facilitate publication, and therefore each chapter has its own, more detailed and specific introduction.

\section{Recruitment}

In Chapter 2, I survey the distribution of juveniles and adult conspecifics in a stagestructured population of a small temperate reef fish (described under 'Study Species' below) to evaluate the influence of resident adults on juvenile abundance. In addition, I examine the potential for adults to influence predator-mediated mortality at settlement. For marine reef fish, strong evidence exists to suggest that post-settlement mortality is often density dependent (Hixon and Carr 1997, Almany and Webster 2006; Jones and McCormick 2006; White et al. 2010; Hixon 2011; Hixon et al. 2012) and appears to be most commonly driven by intraspecific competition for predator refuges at settlement (Anderson 2001; Hixon and Jones 2005). When settlers (recently transitioned larvae) arrive at the reef, they must compete for space, both with other settlers and resident conspecifics (i.e., individuals in older age classes). Weaker competitors may be forced to use marginal habitats or sites where predation risk is greater (e.g. 
Holbrook and Schmitt 2002). Fish in older age classes may be superior competitors (Wilson 2005), and may strongly affect recruitment strength (Tupper and Boutilier 1997; Samhouri et al. 2009), either by excluding settlers from preferred settlement habitats (Ben-Tzvi et al. 2009) or by operating as predators through opportunistic cannibalism (Booman et al. 1991; Smith and Reay 1991). However, the role of older fish may be complicated, due to the fact that established conspecifics can often also increase recruitment success (Lecchini et al. 2007; Hunt et al. 2011), potentially by giving off cues that aid settlers in finding appropriate habitat (Sweatman 1988). If adults increase settler mortality via competition or cannibalism, it can produce delayed density dependence, where population growth $(r)$ is negatively affected by adult abundances at the settlement site based on recruitment from previous years (Pedraza-Garcia and Cubillos 2008). Understanding how older life stages influence recruitment in stage-structured populations can provide insights into how population dynamics may fluctuate over time (e.g., Bjørnstad et al. 1999).

\section{Growth}

In Chapter 3, I assess the application of calcified fish scales as a tool to measure postsettlement growth in reef fish. Somatic growth rates are one of the key life history traits that have important consequences for both individual survival and reproduction (Gotthard et al. 1994; Arendt 1997). However, direct measurement of growth rates in individual fish through time is often logistically difficult or impossible (particularly if one wishes to do this non-destructively). Historical growth rates are often reconstructed from growth increments recorded on calcified structures such as otoliths or scales (Francis 1990; Pierce et al. 1996; Campana and Thorrold 2001; Campana 2005). The successful use of growth increment measurements in otoliths (otolith microstructure) has expanded greatly in recent years (Campana and Thorrold 2001; Campana 2005), but its application has generally been limited to early life history stages (larvae and juveniles) because daily growth increments are difficult to discern and interpret in older fish (Morales-Nin 1988), and moreover, increment deposition rates may become altered (Cermeno et al. 2003), or somatic growth and otolith growth may become decoupled (Klumb et al. 2001). Despite having a reduced temporal resolution relative to otoliths, inter-increment spacing in fish scales may provide an alternative (and potentially more accurate) measure of growth (Klumb et al. 2001). However, this technique has been largely limited to freshwater commercial species 
(salmon - Fisher and Pearcy 1990; Fukuwaka and Kaeriyama 1997; Wells et al. 2003; Fisher and Pearcy 2005; roach - Ibáñez et al. 2008; Britton 2010; perch- Newhard et al. 2012; trout - Beakes et al. 2014). In contrast to otolith increments, which form on a daily cycle (Campana and Thorrold 2001), the deposition of scale increments may vary widely among species (Cheung et al. 2007); therefore, it is necessary to determine the temporal scale as well as the relationship between scale growth and body (somatic) growth for each species. My aim in Chapter 3 is to validate the use of scales as a tool for measuring growth patterns across post-settlement life histories for my study species. This may be useful for future studies that may (i) compare growth rates of samples or populations among sites or seasons (e.g., Marco-Rius et al. 2013) and (ii) examine how previous growth histories affect later life performance, such as reproductive success (e.g., Jennings and Philipp 1992).

\section{Reproduction}

In Chapter 4, I apply the application of scale structures to estimate growth (that I developed in the preceding chapter), and use scale-derived growth estimates to explore how reproductive output in males is affected by growth rates in early life. Individuals that successfully recruit to adulthood may differ in their growth histories, and certain growth histories may be more reproductively successful than others (Donelson et al. 2008). Initial differences in the growth rates of survivors may: (i) persist in the adult population, (ii) become negligible, or (iii) be reversed by compensatory growth later in post-settlement (Sogard and Olla 2002; Hamilton 2008; Sponaugle and Grorud-Covert 2006). Increased growth rates may enlarge adult body size, which is important in determining potential access to mates, oviposition sites, and attractiveness to females (Downhower and Brown 1980; Thompson 1986; Hastings 1988; Côté and Hunte 1989; Oliveira et al. 1999). However, selection pressures on growth rates may fluctuate between life stages (Podolsky and Moran 2006) and lead to trade-offs between life history stages due to opposing selection on traits that minimize mortality during early transition phases (e.g. high growth rates), but also reduce fecundity by compromising later life stage performance (Gimenez et al. 2004; Gagliano et al. 2007). For example, increased foraging time may lower the time available to defend territories, which are vital in procuring mates during spawning (Warner 1987; Hastings and Peterson 2010). We do not yet fully understand how early life history processes affect the reproductive output of individuals that survive to adulthood 
in reef fish (Sponaugle and Grorud-Covert 2006) or how this may interact with male morphological traits (e.g., body size), but if juveniles with a particular growth history have a reproductive advantage as adults, this may indicate that early life histories may determine eventual reproductive output in reef fish.

In Chapter 5, I evaluate how body size influences male reproductive success during a parasitic outbreak. Substantial research across multiple taxonomic groups indicates that parasitemediated sexual selection may strongly modify reproductive success by reducing reproductive output in parasitised males (freshwater fish - Milinski and Bakker 1990; Poulin 1994; birds- Zuk et al. 1990; mammals- Ehman and Scott 2002; invertebrates -Webster et al. 2003). Despite the high ectoparasite prevalence in reef fish (Rohde et al. 1995) and the deleterious effects of parasite infection on host fitness (Adlard and Lester 1994; Finley and Forrester 2003; Binning et al. 2013), the role of parasitism in determining reproductive success in reef fish has been largely unexplored. Parasite abundance and diversity appear to increase with body size in reef fish (Grutter 1994; Rohde et al. 1995; Lo et al. 1998), suggesting that parasitism may select for smaller body size, while sexual selection (intra-and intersexual) may select for larger body size, potentially leading to a conflict between selection mechanisms (Cable and van Oosterhout 2007). If selection against parasitized males is strong, parasite infection may have important implications for effective population sizes, genetic diversity and the evolutionary trajectories for populations over time (Hamilton 1982).

Finally in Chapter 6, I measure how larval mortality varies among source populations, to understand how reproductive success is potentially shaped by attributes of the parental population. During dispersal, larvae from separate source populations may mix in the water column and consequently, larvae replenishing settlement sites may be sourced from multiple populations (Cowen et al. 2006). If certain sites produce larvae that have lower larval mortality rates, more of their larvae will survive pelagic dispersal and consequently, those sites will contribute more to metapopulation dynamics (groups of interconnected subpopulations). Current population connectivity models assume that larval mortality is equal among sub-populations (e.g. Cowen et al. 2000; James et al. 2002), and do not account for the potential effects of differential larval mortality among larval sources. Mortality after hatching is typically high (Fortier and Leggett 1985; Houde 1989), but the potential for larval mortality to differ between source 
populations has rarely been evaluated. Larval mortality in fish appears to be driven heavily by starvation (Hjort 1914; Cushing 1975) and larvae from separate natal reefs may vary in their starvation resistance if their source populations differ in age/size structure (e.g., Berkeley et al. 2004) or environmental variables, such as temperature (e.g., Mendiola et al. 2007). Understanding how differences in mortality between source populations may influence population connectivity has important implications for a variety of processes including the persistence and extinction of local populations, species conservation, and marine reserve placement (Jones et al. 2009; Cowen and Sponaugle 2009; Almany et al. 2009; Planes et al. 2009).

\section{Study species}

All of the research for this thesis was conducted on the temperate reef fish, Forsterygion lapillum Hardy, 1989 (the common triplefin). F. lapillum is a small (maximum total length 70 $\mathrm{mm}$ ) blennioid fish (blennies) of the Family Tripterygiidae (triplefins). Blennies are extremely diverse and well represented on both tropical and temperate reefs (Hastings 2009), and $F$. lapillum is one of the most common benthic fish in shallow coastal areas around New Zealand (Willis 2001; Wellenreuther et al. 2007a; Wellenreuther et al. 2008). Larvae hatch from benthic eggs and remain in the pelagic stage for a period of 1-3 months (Shima and Swearer 2009a; Kohn and Clements 2011), before settling into macroalgal habitats as juveniles (NovemberMarch, McDermott and Shima 2006). F. lapillum is most commonly associated with sheltered cobble habitats (Syms 1995; Feary and Clements 2006; Wellenreuther and Clements 2008), although individuals may use a variety of substrate types (Feary and Clements 2006). Juveniles and adults appear to use similar substrate types (Syms 1995; Wellenreuther and Clements 2008), but preferences for particular algal habitats may differ between juveniles and adults (McDermott and Shima 2006; Wellenreuther and Clements 2008). F. lapillum exhibits high levels of site fidelity throughout ontogeny (Shima et al. 2012); adults rarely move further than a few meters (Clements 2003).

F. lapillum is an asynchronous continuous spawner, that may lay eggs daily (Warren 1990) throughout the breeding season (September-December in my study area). During the breeding season, males are typically site attached (Clements 2003), assume dark black nuptial colours (Wellenreuther \& Clements 2007), display elaborate courtship behaviour (Handford 
1979), and defend breeding territories within cobble habitats (Warren 1990). Unlike males, females move greater distances during the breeding season to find mating opportunities (Clements 2003) and typically lay eggs underneath cobbles within the territory of males (Feary and Clements 2006). Males remain close to the nest entrance during defense (Warren 1990) and may defend clutches of eggs deposited by multiple females, with larvae hatching after approximately 3 weeks (Francis 2012).

\section{Study location}

This thesis was conducted in the Wellington region in New Zealand, at sites within Wellington harbour and along the more exposed Wellington south coast (Fig. 1.1). These sites were typically shallow subtidal ( 0-3 m depth), relatively sheltered, and typically characterized by a mix of substrates, but usually dominated by cobble reef. The Wellington harbour is a large embayment $\left(87 \mathrm{~km}^{2}\right.$, Heath 1985) that is partially isolated from outside oceanic flows (Booth 1975). The harbour area is typically 1-2 degrees $C$ warmer on average than the exposed southern coast, and contains higher levels of organic matter as well as nitrogen (Helson et al. 2007). The harbour is also sheltered from strong southerly swells, but is exposed to high northerly winds. In contrast, the exposed southern coast is a relatively high-energy coastline, characterized by low productivity and large swells from southerly storms (Bowman et al. 1983). Previous work conducted on $F$. lapillum in this geographic area suggests that larval phenotypes (Shima and Swearer 2009b), selective mortality among settlers (Smith and Shima 2011), as well as juvenile and adult densities (Pérez-Matus and Shima 2010) all differ between the harbour and the exposed southern coast. Larval retention within the harbour is presumed to be high, although exchange is sufficiently high such that the majority of settlers on the south coast may be spawned from within the harbour (Swearer and Shima 2009). 

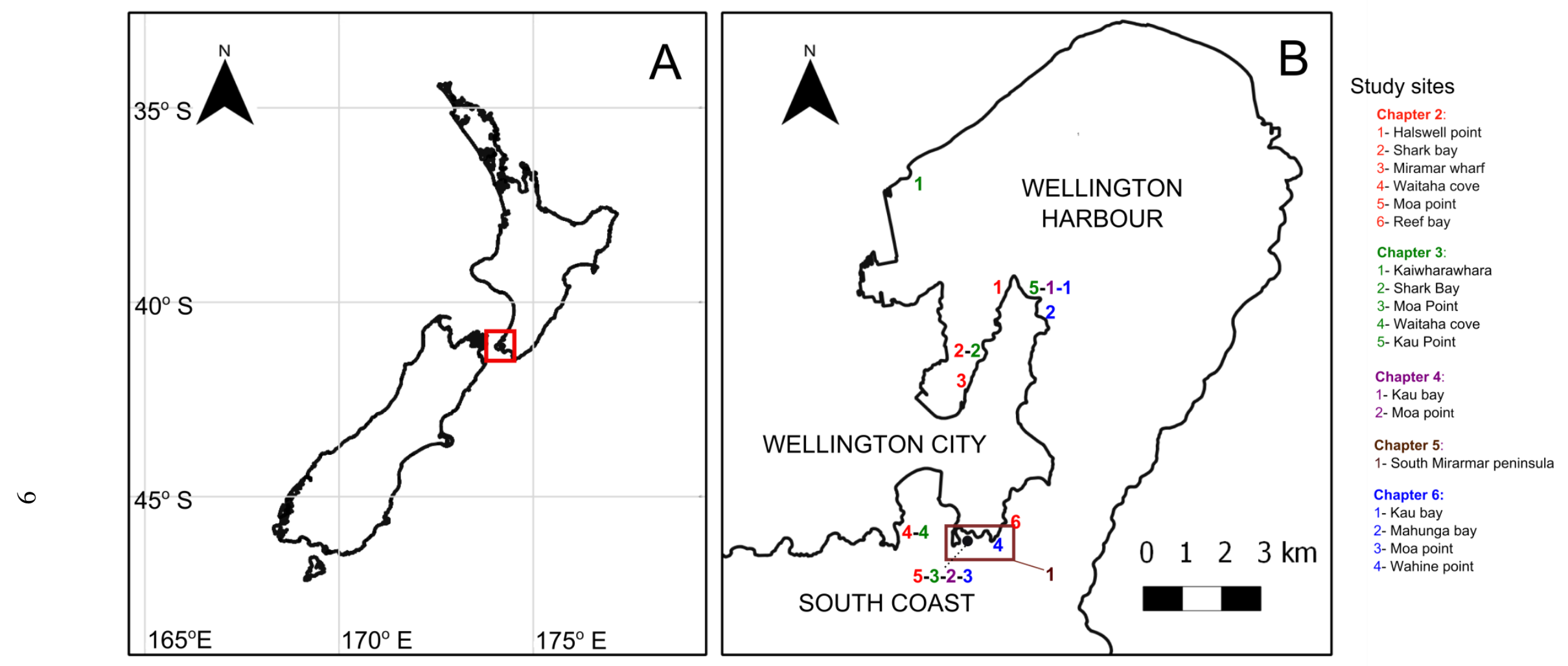

Figure 1.1 Red box within panel A shows the location of the study site within New Zealand, enlarged in panel $\mathbf{B}$, with the specific study areas for each chapter indicated by a colour-coded number. 


\section{Questions and implications}

Overall, current research suggests that processes at multiple life stages may influence population dynamics in reef fish. Nevertheless, the relative importance of different mechanisms underlying these processes across life histories remains largely unexplored. In the following chapters, I address five main questions about processes occurring throughout ontogeny in the common triplefin:

1. How do older life stages affect recruitment in stage-structured populations and determine spatio-temporal variation in recruitment success across habitats (Chapter 2)?

2. Among surviving individuals, how can we measure previous growth histories (Chapter $3)$ ?

3. How does prior growth history, in combination with morphological traits, determine individual variation in male reproductive output (Chapter 4)?

4. How important is parasitic infection in modifying established patterns of individual reproductive success among males (Chapter 5)?

5. Finally, does early larval mortality rate vary as a function of source population (Chapter 6)?

By integrating multiple processes (recruitment, growth and reproduction) and including explicit investigations of stage-structured interactions, we can gain a more complete understanding of the drivers of reef fish population dynamics. 
Chapter 2 - Patterns of co-occurrence and interactions between age classes of the common triplefin, Forsterygion lapillum 


\subsection{Abstract}

Many marine organisms have pelagic larval stages that settle into benthic habitats occupied by older individuals; however, a mechanistic understanding of intercohort interactions remains elusive for most species. Patterns of spatial covariation in the densities of juvenile and adult age classes of a small temperate reef fish, the common triplefin (Forsterygion lapillum), were evaluated during the recruitment season (Feb-Mar, 2011) in Wellington, New Zealand $\left(41^{\circ} 17^{\prime} \mathrm{S}, 174^{\circ} 46^{\prime} \mathrm{E}\right)$. The relationship between juvenile and adult density among sites was best approximated by a dome-shaped curve, with a negative correlation between densities of juveniles and adults at higher adult densities. The curve shape was temporally variable, but was unaffected by settlement habitat type (algal species). A laboratory experiment using a "multiple predator effects" design tested the hypothesis that increased settler mortality in the presence of adults (via enhanced predation risk or cannibalism) contributed to the observed negative relationship between juveniles and adults. Settler mortality did not differ between controls and treatments that contained either one $(\mathrm{p}=.08)$ or two $(\mathrm{p}=.09)$ adults. However, post hoc analyses revealed a significant positive correlation between the mean length of juveniles used in experimental trials and survival of juveniles in these treatments, suggesting that smaller juveniles may be vulnerable to cannibalism. There was no evidence for risk enhancement or predator interference when adults were present alongside a heterospecific predator (Forsterygion varium). These results highlight the complex nature of intercohort relationships in shaping recruitment patterns, and add to the growing body of literature recognizing the importance of age class interactions. 


\subsection{Introduction}

A central goal of marine ecologists is to determine the processes that contribute to variation in recruitment, and how this variation, in turn, shapes the abundance, dynamics, and spatial distribution of organisms (Roughgarden et al. 1988; Caley et al. 1996). For reef fish, there is strong empirical evidence that intraspecific interactions between recent settlers can result in density dependent mortality, which may modify recruitment patterns (Hixon and Jones 2005; Almany and Webster 2006; Jones and McCormick 2006; White et al. 2010; Hixon 2011; Hixon et al. 2012). Many studies have focused on interactions between individuals settling in the same recruitment period (within-cohort interactions); however, recently recruited fish may frequently co-occur (and potentially compete) with adults from previous cohorts (intercohort interactions). Adults are typically larger, and due to size-based competitive asymmetries, larger conspecifics may exert a strong competitive effect on settlers (Webster and Hixon 2000; Wilson 2005), which can lead to density dependent mortality (Wilson and Osenberg 2002; Webster 2004; Wilson 2005; Samhouri et al. 2009). There is also evidence that established adults may increase settler mortality by functioning as opportunistic cannibals (Smith and Reay 1991). Despite the growing evidence that intercohort interactions can mediate recruitment dynamics, the effects of intercohort interactions remain relatively unexplored in reef fish (Samhouri et al. 2009).

The effects of adult conspecifics on recruitment patterns may be complex, due to the fact that adults can have both positive and negative effects on younger cohorts (Wilson and Osenberg 2002; Adam 2011). For instance, adults may provide cues that enable settlers to find appropriate habitats (Sweatman 1988; Stamps and Krishnan 2005). Alternatively, competition between adults and juveniles can lead to the competitive exclusion of juveniles from preferred habitats, and this can make settlers more vulnerable to predation (Tupper and Boutilier 1995; Bjørnstad et al. 2004; Wilson 2005; Forrester et al. 2006). The competitive effects of resident adults may be further exacerbated by the presence of priority effects, whereby the intensity of competition is increased for settlers who arrive into a habitat with prior residents (Geange and Stier 2009). Furthermore, adults may act simultaneously as both competitors and predators if adults cannibalize settlers (Polis et al. 1989; Schmitt et al. 2009). If adults increase settler mortality via competition or cannibalism, they may strongly influence yearly recruitment patterns (Bjørnstad 
et al. 1999; Bjørnstad et al. 2004) by suppressing the recruitment of future cohorts (delayed density dependence, Bjørnstad et al. 2004; Pedraza-Garcia and Cubillos 2008).

Settler survival during settlement is strongly shaped by habitat characteristics for many reef fishes (Beukers and Jones 1998; Johnson 2007), and the type or quality of settlement habitat may strongly influence the relative importance of intercohort interactions by mediating both competitor and predator-prey relationships (Persson et al. 2000; Wilson 2005; Samhouri et al. 2009; Adam 2011). Previous studies have found that habitat can strongly affect intercohort interactions; however, these studies have mainly focused on the influence of habitat availability (rock number - Samhouri et al. 2009, crevice size and number - Adam 2011), or larger scale differences in habitat use (e.g., distance from shore - Persson et al. 2000). The effect of finescale differences in settlement habitat types on intercohort dynamics is currently unknown. For instance, juveniles of many temperate reef fish rely heavily upon macroalgal habitats at settlement (Holbrook et al. 1990; Carr 1994) with research suggesting that the identity of macroalgae can strongly influence variability in the recruitment of reef fish (Jones 1984; Holbrook et al. 1990; Levin 1991; Anderson and Millar 2004) via differential/selective survival of settlers in different algal types (Connell and Jones 1991; Tupper and Boutilier 1997).

Morphological variation among algal species may provide different levels of habitat 'complexity' for reef fish (Pérez-Matus \& Shima, 2010), and by extension, the habitats afforded by different species of algae may alter the intensity of intercohort interactions by (i) modifying intercohort competition for space and (ii) altering both predator-induced mortality and cannibalism on recently settled fish. If the nature of intercohort interactions varies between algal species and intercohort interactions have a strong effect on juvenile abundance, then the distribution of algal species may contribute strongly to spatial variation in recruitment.

Here, I investigate intercohort interactions for the temperate reef fish, Forsterygion lapillum (the common triplefin). F. lapillum is a small reef fish (maximum total length $70 \mathrm{~mm}$ ) of the Family Tripterygiidae (triplefins), and one of the most common benthic fish in shallow coastal areas around New Zealand (Willis 2001; Wellenreuther et al. 2007a; Wellenreuther et al. 2008). Larvae hatch from benthic eggs and remain in the pelagic stage for a period of 1-3 months (Shima and Swearer 2009a; Kohn and Clements 2011), before settling into macroalgal habitats as juveniles (November-March, McDermott and Shima 2006). F. lapillum is most commonly 
associated with sheltered cobble habitats (Syms 1995, Feary and Clements 2006, Wellenreuther and Clements 2008), although individuals may utilize a variety of substrate types (Feary and Clements 2006). Juveniles and adults appear to use similar substrate types (Syms 1995; Wellenreuther and Clements 2008), but preferences for particular algal habitats may differ between juveniles and adults (McDermott and Shima 2006; Wellenreuther and Clements 2008). F. lapillum exhibits high levels of site fidelity throughout ontogeny (Shima et al. 2012); adults rarely move further than a few meters (Clements 2003), and juveniles appear to maintain small home ranges $\left(500-1000 \mathrm{~cm}^{2}\right)$. The high degree of overlap in habitat use and site fidelity suggest that intercohort interactions may play an important role in the recruitment dynamics of this species, and previous work has indicated that intercohort effects may be present (Wellenreuther 2007).

Intercohort interactions may be inferred from observed patterns of variation in density between juveniles and adults (e.g., Webster 2003). Based on this putative relationship, several competing hypotheses can be formulated and differentiated from one another (Table 2.1). I evaluate the nature of intercohort interactions for F. lapillum empirically by (1) establishing that adults and juveniles co-occur over a small spatial scale (and therefore have the potential to interact), and (2) characterising the pattern of variation between juveniles and adults. Furthermore, I examine how the correlation between juveniles and adults is modified by two different algal types commonly found in F. lapillum habitats: Carpophyllum maschalocarpum and Cystophora retroflexa. I compare the densities of juvenile and adult F. lapillum between monospecific $C$. maschalocarpum stands and mixed stands (those containing both $C$. retroflexa and C. maschalocarpum) because previous studies indicate that (i) selective mortality may be higher in C. maschalocarpum stands (Smith and Shima 2011), (ii) individuals may experience faster post-settlement growth in mixed stands compared with C. maschalocarpum only stands (Smith and Shima 2011) and (iii) the presence of Cystophora may increase recruitment, potentially because settlers preferentially select Cystophora habitats during settlement (McDermott and Shima 2006). I therefore hypothesize that the spatial co-occurrence between juveniles and adults may also differ between these habitat types. To assess the generality of these patterns, I conduct this study within two distinct regions: an enclosed harbour and an exposed coast. Finally, (3) I use a laboratory experiment to test the hypothesis that cannibalism and/or multiple-predator effects between a conspecific and a heterospecific predator (both 
hypotheses motivated by my empirical observations) increase the mortality of recently settled fish and are potential mechanisms that may account for the variation between juvenile and adult densities that I observed in the field. 
Table 2.1 Hypothetical relationships between juvenile densities (y axis) and adult densities ( $\mathrm{x}$ axis), and the underlying processes that may be responsible for each relationship (assuming overlap in habitat use between age classes occurs). The type of settlement pattern required for each process is described as (i) selective - settling larvae display habitat preferences and are able to choose settlement habitats, (ii) passive - settling larvae are unable to select specific settlement habitats and settle randomly, or (iii) either- the process can occur regardless of whether or not settlement is selective or passive. In addition, the possibility that a particular process could lead to intercohort density dependence (DD) is indicated by a yes (Y) or a no (N). In some cases (marked with an *), density dependence could only occur if (i) settlement is selective and (ii) there is lower survival in the selected habitat. The authors acknowledge that this is not an exhaustive list of potential hypotheses, but rather a list of observed processes in the literature

\begin{tabular}{|c|c|c|c|c|c|}
\hline \multicolumn{2}{|r|}{ Expected relationship } & Potential processes & $\begin{array}{l}\text { Passive or } \\
\text { selective }\end{array}$ & DD & Literature \\
\hline \multirow{3}{*}{ 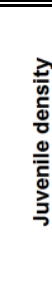 } & \multirow[b]{3}{*}{ Adult density } & No effect of adult conspecifics & Either & $\mathbf{N}$ & Levin 1993 \\
\hline & & $\begin{array}{l}\text { Passive (non-selective) settlement } \\
\text { of settlers across habitats }\end{array}$ & Passive & $\mathbf{N}$ & Forrester 1999 \\
\hline & & $\begin{array}{l}\text { Sub-lethal effects of adults on } \\
\text { settler growth and maturity }\end{array}$ & Either & $\mathbf{N}$ & $\begin{array}{c}\text { Jones } 1987, \\
\text { Tupper and } \\
\text { Boutilier } 1995\end{array}$ \\
\hline \multirow{3}{*}{ 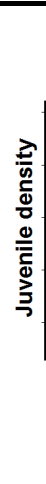 } & \multirow[b]{3}{*}{ Adult density } & $\begin{array}{l}\text { Settlers use resident adults as } \\
\text { settlement cues }\end{array}$ & Selective & $\mathbf{N}$ & $\begin{array}{c}\text { Hunt 2011, } \\
\text { Stamps and } \\
\text { Krishnan 2005 }\end{array}$ \\
\hline & & $\begin{array}{l}\text { Juvenile recruitment success } \\
\text { predicts future adult distributions } \\
\text { (i.e. densities are correlated within } \\
\text { habitat patches from year to year) }\end{array}$ & Either & $\mathbf{N}$ & Gutiérrez 1998 \\
\hline & & $\begin{array}{l}\text { Co-variation of settler habitat } \\
\text { quality and adult density }\end{array}$ & Either & $\begin{array}{c}\mathrm{Y} \\
\text { (cryptic } \\
\text { DD) } / \mathrm{N}\end{array}$ & $\begin{array}{c}\text { Shima and } \\
\text { Osenberg } 2003\end{array}$ \\
\hline \multirow{3}{*}{ 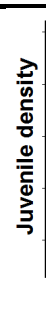 } & \multirow[b]{3}{*}{ Adult density } & $\begin{array}{l}\text { Competitive exclusion from habitats } \\
\text { by adults }\end{array}$ & Either & $\mathbf{Y}$ & $\begin{array}{c}\text { Samhouri 2009, } \\
\text { Webster } 2004\end{array}$ \\
\hline & & Cannibalism & Either & $\mathbf{Y}$ & Bjørnstad 1999 \\
\hline & & $\begin{array}{l}\text { Settlers shift habitat preferences as } \\
\text { adult density increases to avoid } \\
\text { settling near adults }\end{array}$ & Selective & $\mathbf{Y} * \mathbf{N}$ & Ayllón 2012 \\
\hline \multirow{2}{*}{ 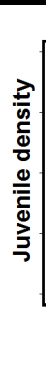 } & \multirow[b]{2}{*}{ Adult density } & $\begin{array}{l}\text { Combination of facilitation by prior } \\
\text { residents or co-variation in habitat } \\
\text { quality (positive) combined with } \\
\text { competition/cannibalism (negative) }\end{array}$ & Either & $\mathbf{Y}$ & Adam 2011 \\
\hline & & $\begin{array}{l}\text { Facilitation by prior residents or co- } \\
\text { variation in habitat quality } \\
\text { (positive) combined with } \\
\text { differential habitat selection by } \\
\text { settlers at high adult densities }\end{array}$ & Selective & $\mathbf{Y} * / \mathbf{N}$ & - \\
\hline
\end{tabular}




\subsection{Methods}

\subsubsection{Density surveys}

I surveyed fish across a set of locations to determine how the density of juvenile $F$. lapillum co-varies with the density of adults. I repeated these surveys to evaluate temporal consistency in patterns over short time scales (i.e., the robustness or repeatability of the correlation), and the statistical model also evaluated how any relationships between juveniles and adults might be mediated by (i) macroalgal identity and (ii) recruitment location (harbour or exposed coast). I conducted this study in the coastal waters around the Wellington region in New Zealand, at sites within Wellington harbour and along the more exposed Wellington south coast. The Wellington harbour is a large embayment $\left(87 \mathrm{~km}^{2}\right.$, Heath 1985) that is partially isolated from outside oceanic flows (Booth 1975). The harbour area is typically 1-2 degrees warmer on average than the exposed southern coast, and contains higher levels of organic matter as well as nitrogen (Helson et al. 2007). The harbour is also sheltered from strong southerly swells, but is exposed to high northerly winds. In contrast, the exposed southern coast is a relatively high-energy coastline, characterized by low productivity and large swells from southerly storms (Bowman et al. 1983). Previous work conducted on F. lapillum in this geographic area suggests that larval phenotypes (Shima and Swearer 2009b), selective mortality among settlers (Smith and Shima 2011), as well as juvenile and adult densities (Perez-Matus and Shima 2010) all differ between the harbour and the exposed southern coast. Larval retention within the harbour is presumed to be high, although exchange is sufficiently high such that the majority of settlers on the south coast may be spawned from within the harbour (Swearer and Shima 2009).

I conducted surveys in sheltered bays on rocky reef habitats that contained large stands of macroalgae, a common settlement habitat for F. lapillum (Feary and Clements 2006; McDermott and Shima 2006). I selected sites non-randomly, based upon the presence of two common fucoid algal species, Carpophyllum maschalocarpum and Cystophora retroflexa, both known to be used by recently settled F. lapillum (McDermott and Shima 2006). Specifically, I surveyed six different sites (small bays between 150-250 m in width) around the Miramar Peninsula that were

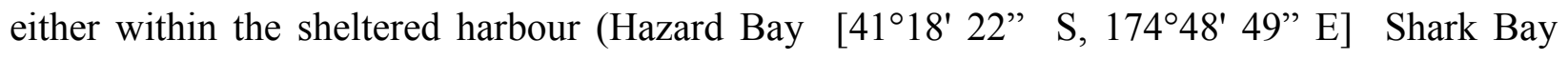




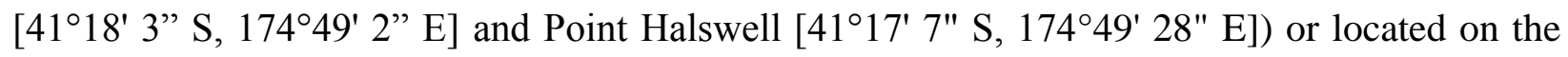

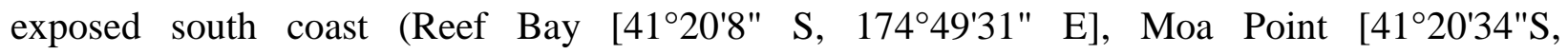
$174^{\circ} 48^{\prime} 38^{\prime \prime E}$, and Waitaha Cove [41 $\left.20^{\circ} 28^{\prime \prime S}, 174^{\circ} 47^{\prime} 32^{\prime \prime E}\right]$ ).

At each site, I established $4 \mathrm{~m} \times 10 \mathrm{~m}$ transects $(\mathrm{n}=6)$ within macroalgal stands along contiguous areas of rocky reef running parallel to shore at an approximate depth contour of 2-3 m. All transects were haphazardly placed (i.e., without any formal method of randomization) within the macroalgal stands (maintaining a minimum distance of $10 \mathrm{~m}$ between transects). Within each transect, I selected and marked (using a small float adjacent to the sampling area) two permanent $1 \mathrm{~m}^{2}$ quadrats ( $\mathrm{n}=12$ quadrats per site). These quadrats were positioned such that they were separated from one another by $\geq 3$ meters, and they were selected non-randomly such that each transect had one quadrat that was comprised of $\sim 100 \%$ C. maschalocarpum and a second quadrat that was comprised of a mixture of C. maschalocarpum and C. retroflexa ( $\sim 50 \%$ cover of each species). I led dive teams that repeated surveys over 3 distinct sampling periods at roughly 2-week intervals from February to March 2011, during the peak recruitment season for F. lapillum (Wellenreuther and Clements 2008). Sampling of harbour and coast locations were paired in time as far as weather and sea conditions would allow (harbour - 9 Feb, 18 Feb, 9 Mar; south coast - $10 \mathrm{Feb}, 20 \mathrm{Feb}, 3 \mathrm{Mar}$ ). To minimise the potential introduction of systematic bias, I varied the order that I sampled individual sites and transects within locations among sampling dates. All surveys were conducted with the aid of SCUBA. At each quadrat, dive teams exhaustively searched from the top of the macroalgal canopy down to the underlying substrate for all $F$. lapillum. All fish within the quadrat were counted and classified as either juveniles $(<40 \mathrm{~mm})$ or adults $(>40 \mathrm{~mm})$. Search times were approximately $1-2$ minutes; however, no maximum time was specified prior to surveys. F. lapillum settles at $\sim 26 \mathrm{~mm}$ (see Experimental set-up and trials below); therefore, the juvenile size category included both recently settled fish and older juveniles. Surveyed adults may have settled earlier within the same recruitment season (austral summer 2011), or settled in the previous recruitment season (austral summer 2010).

\subsubsection{Statistical models and data analysis of density surveys}


I conducted repeated measurements on the same sampling units over time in order to investigate the repeatability and robustness (short-time scale variability) of any relationship between densities of juveniles and adults. Due to the non-independence of data points taken on the same sampling units (quadrats), I used a mixed effects model to account for correlations among repeated measurements by fitting a random effect of the repeated measurement (Bolker et al. 2009). Therefore, I included a unique number for each quadrat (quadrat ID), 1-72, as a random intercept effect to account for repeated measurements on quadrats over time.

I used a series of generalized linear mixed models (GLMM's) fitted with a Poisson error structure to (1) describe the broad scale differences of juvenile and adult density between sites and algal species and (2) examine how adult density, macroalgal identity, and recruitment location (harbour or south coast) affect juvenile density over time.

Density patterns among sites, macroalgal species, and sampling periods

To evaluate patterns of variation in the density of juveniles and adults among sites and algal types over time, I fit two GLMM's (I analysed adult density and juvenile density as response variables in two separate models). I included site, algal identity, and sample period as fixed factors and quadrat ID as a random factor. To test the effect of the fixed factors (site, macroalgal identity, and sampling date) on juvenile and adult density, I compared nested models with and without each factor using a likelihood ratio test. I inferred statistical significance of the main effects if the model including the fixed factor was significantly different from the one without the fixed factor.

\section{Relationship between juvenile and adult densities}

In order to make comparisons between models supporting different hypotheses, I employed a model selection process by using Akaike Information Criterion (AIC, Akaike 1974) to rank a candidate set of biologically relevant models, a widely recommended practice for ecological data (Johnson and Omland 2004). As preliminary analyses indicated that the relationship between juvenile and adult density was potentially curvilinear, I included a second- 
order polynomial term of adult density (i.e., a quadratic fit) in order to test for curvature in the relationship between juveniles and adults (I evaluated whether the second-order term for adult density was significantly different from zero, and interpreted this as a significant departure from linearity). Firstly, I created a "global model", which included all the fixed factors and interaction terms that were of interest. Specifically, I included adult density, the second-order term of adult density, macroalgal identity, recruitment location (harbour or south coast), and sampling period as fixed factors. In addition, I included 6 two-way interaction terms (3 main factors crossed with both polynomial terms of adult density) in order to test how the effects of adult density on juvenile density vary between algal species (adult density $\times$ macroalgal identity, second-order adult density $\times$ macroalgal identity), recruitment locations (adult density $\times$ recruitment location, second-order adult density $\times$ recruitment location) and sampling periods (adult density $\times$ sampling period, second-order adult density $\times$ sampling period). All input variables (adult density, macroalgal identity, recruitment location, and sampling date) were centred in order to: (i) allow for the interpretation of main effects in the presence of interaction terms, (ii) reduce collinearity between the linear and second-order terms of adult density, and (iii) make regression coefficients more interpretable (Schielzeth 2010). Adult density was centred with a mean of zero and a standard deviation of 0.5 , in order to make comparisons between effect sizes of continuous variables (adult density) and categorical variables easier to interpret (Gelman and Hill 2007). Each model also included a random intercept term for site, because I selected sites from a pool of possible sites within each location (harbour and south coast). Furthermore, I was primarily interested in the overall effects of adult density on juvenile density, rather than variability in these effects between individual sites. Using all of the fixed factors and interactions mentioned above, I created a candidate set of all possible models while excluding any models where the higher second-order term of adult density appeared without the linear term (160 candidate models in total). I used AICc values to assess model fits and rank the candidate model list because they are more appropriate than AIC for model selection with smaller sample sizes. Several models had $\triangle \mathrm{AICc}<2$, suggesting that there was high support for multiple models (Burnham and Anderson 2002). Therefore, I used a model averaging approach to make inferences across a selected group of top models by obtaining averaged parameter estimates for the fixed factors (see Appendix A). To select a group of top models for model averaging, I used a cut-off criterion of $\Delta$ AICc $<2$ (Burnham and Anderson 2002; Grueber et al. 2011). Once 
model averaged parameters were obtained, I used Wald Z-scores to make inferences about each parameter estimate.

I tested each of the models for overdispersion by calculating a goodness of fit test on the global model (including all fixed factors before model selection, Anderson and Burnham 2002, pg. 305). I compared the expected Pearson $X^{2}$ distribution (given the residual sum of squares and the residual degrees of freedom) to the actual model using a goodness of fit test (Hayes et al. 2003; Bolker et al. 2009). The calculated overdispersion parameter showed no evidence for overdispersion $\left(X^{2}=200.74\right.$, residual degrees of freedom $\left.[R D F]=199, p=0.452\right)$.

The initial analysis indicated that the effects of adults on juveniles might be variable over time; therefore, in addition to analyzing the full model mentioned above, I ran a separate analysis for each sampling period. I created three global models (one for each sampling period) that included adult density, second-order adult density, recruitment location, and macroalgal identity as fixed effects as well as 4 two-way interaction terms (adult density $\times$ macroalgal identity, second-order adult density $\times$ macroalgal identity, adult density $\times$ recruitment location, secondorder adult density $\times$ recruitment location). Each of the three global models included a random intercept term of site; however, as these models were comparing within sampling periods and therefore not using repeated measurements on sampling units, they did not require the use of a random effect for quadrat ID. I generated a candidate list of models for each global model, again excluding any models where the second-order term for adult density was present without the linear term of adult density (38 models total). As before, there was support for more than one model; therefore, I conducted model averaging. I tested for overdispersion on each of the global models as before, and found that the sample 1 model was not overdispersed $\left(X^{2}=72.16\right.$, $\mathrm{RDF}=62, \mathrm{p}=0.177)$; however, overdispersion was evident in the sample 2 model $\left(\mathrm{X}^{2}=95.922\right.$, $\mathrm{RDF}=62, \mathrm{p}=0.003)$, and the sample 3 model $\left(\mathrm{X}^{2}=100.206, \mathrm{RDF}=62.00, \mathrm{p}=0.002\right)$. For sample model 1 , I used the same model cut-off criteria as described before $(\Delta \mathrm{AICc}<2)$. For sample model 2 and sample model 3, I ranked candidate model sets using quasi AICc values (QAICc), which account for problems associated with model selection in overdispersed data sets (Richards 2008). As with AICc values, I used a cut-off of $\Delta$ QAICc $<2$ for selection of a top model set. 
As results suggested that the relationship between juvenile and adult density was curvilinear, I further explored how juvenile density responds to adult density by estimating the curve peak (i.e. the adult density where juvenile density is at its highest). For the quadratic curve, the adult density that correlates to the peak value of juvenile density (i.e. the point after which juvenile density decreases with increasing adult density) can be calculated using the following formula:

$$
\text { Peak value }=\frac{-b}{2(a)}
$$

Where $b$ is the regression coefficient for the linear term of adult density and $a$ is the regression coefficient for the quadratic term. Because there was a significant interaction between the second-order term of adult density and sampling date, a global peak value (i.e. pooling across sampling dates) for juvenile density would be inappropriate because the peak values for each sampling period would vary significantly. Therefore, I calculated estimates for the curve peak for each sampling point separately. I used the Delta method (as per Hirschberg and Lye 2005) to estimate the peak values and confidence intervals around the peaks.

\subsubsection{Experimental test of multiple-predator effects and cannibalism}

I conducted an experiment to investigate and differentiate between several potential underlying mechanisms that might account for the apparent effect of intercohort interactions on juvenile density (inferred from the observational component of this study, see Results). Specifically, my experimental design allowed us to determine whether the presence of adult $F$. lapillum affects settler mortality via (i) cannibalism, (ii) synergistic predator interactions, and/or (iii) competition that increases risk of predation by another species (only testable in the absence of cannibalism). In order to accomplish this, I used a modified multiple predator effects design (MPE, Sih et al. 1998, Griffen 2006) to simultaneously quantify the separate and combined effects of two potential predators (adult F. lapillum and the heterospecific predator Forsterygion 
varium) on recently settled fish (approximately 7-10 d post-settlement, Fig. 2.1A). I used $F$. varium (Fig. 2.1A) as a predator species because it frequently co-occurs with F. lapillum in the coastal areas around Wellington (Pérez-Matus and Shima 2010) as well as other parts of New Zealand (Feary and Clements 2006), and will readily consume settlers in the laboratory (PJM personal observation). I used recent settlers (<30 mm in length) in my laboratory experiment because the majority of post-settlement mortality is thought to occur during this life stage in reef fish (Almany and Webster 2006). In the absence of cannibalism, MPE designs can detect competitive interactions (e.g., between adults and juveniles of $F$. lapillum in this case) that may increase mortality due to predation from other predators (i.e., where competition, rather than consumption by adults causes increased settler mortality, Schmitt et al. 2009). Multiple predator effects designs determine the effects of two species foraging simultaneously, by estimating the expected prey survival based upon the independent risk posed by each species in isolation. Specifically, MPE models aim to ascertain if the presence of multiple species results in 'emergent effects', whereby the combination of species either reduces predation risk (e.g., through predator-predator interference) or enhances predation risk (e.g., predator facilitation or competition with prey) from what would be expected from both predators foraging independently (Sih et al. 1998). For this experiment, I used both the additive and substitutive MPE experimental designs (Table 2.2) because I was interested in both interspecific (between adult $F$. lapillum and $F$. varium predators, additive design) and intraspecific predator interactions (within adult $F$. lapillum and within $F$. varium, substitutive). For both the additive and substitutive designs, the expected values (based on the species foraging in isolation) are compared with the observed values to determine if emergent effects are present. If observed survival is higher than expected survival, then there is evidence for risk reduction; alternatively, if survival in the observed treatment is lower than expected, then the hypothesis of risk enhancement is supported.

Given that estimated prey mortality in the combined predator treatment may exceed $100 \%$ (e.g. when the number of prey consumed by both predators in isolation is greater than the total number of prey in the multi-species treatment), non-additive calculations are used to determine expected prey survival (Sih et al. 1998). In order to account for the differences in 
predator densities between additive and substitutive designs, expected mortality is calculated differently for each design. The expected survival for an additive model, in the absence of emergent effects, can be determined by (Griffen 2006):

$$
E_{\text {add }}=\frac{N_{\text {predator } 1} \times N_{\text {predator } 2}}{N_{\text {control }}}
$$

Where $E_{\text {add }}$ is the expected survival, $N_{\text {predatorl }}$ is number of prey surviving in a tank containing only one adult $F$. lapillum, $N_{\text {predator } 2}$ is the number of prey surviving in the tank containing a single $F$. varium, and $N_{\text {control }}$ is the number of prey surviving in the tank with no conspecific adults or predators (background mortality). If the control tank has zero deaths, then the expected value for the control is calculated without a denominator. For the substitutive design, expected survival in the absence of emergent effects can be calculated by (Griffen 2006):

$$
E_{\text {sub }}=\frac{\left(N_{\text {predator } 1} \times N_{\text {predator } 2}\right)}{2}
$$

Where $E_{\text {sub }}$ is the expected survival under a substitutive design, $N_{\text {predatorl }}$ is the number of surviving prey in the tank containing two adult $F$. lapillum, and $N_{\text {predator } 2}$ is the number of prey items surviving in the tank containing two $F$. varium. 
Table 2.2 Experimental treatments for laboratory experiment including additive and substitutive designs along with the corresponding process they aimed to quantify

\begin{tabular}{|c|c|c|c|c|}
\hline Treatment & $\begin{array}{l}\text { Predator } \\
\text { density }\end{array}$ & $\begin{array}{c}\text { Multiple } \\
\text { predator effects } \\
\text { design }\end{array}$ & Species & Process tested \\
\hline Control & 0 & Both & None & Background mortality \\
\hline Adult & 1 & Additive & Forsterygion lapillum & Cannibalism \\
\hline 2 adults & 2 & Substitutive & 2 Forsterygion lapillum & $\begin{array}{l}\text { Intraspecific interference or } \\
\text { enhancement of cannibalism } \\
\text { mortality between adults }\end{array}$ \\
\hline $\begin{array}{l}\text { Predator \& } \\
\text { adult }\end{array}$ & 2 & Both & $\begin{array}{l}\text { Forsterygion lapillum } \\
\qquad \& \\
\text { Forsterygion varium }\end{array}$ & $\begin{array}{l}\text { Interspecific interference or } \\
\text { risk enhancement }\end{array}$ \\
\hline Predator & 1 & Additive & Forsterygion varium & Predation \\
\hline 2 predators & 2 & Substitutive & 2 Forsterygion varium & $\begin{array}{l}\text { Intraspecific interference or } \\
\text { enhancement between } \\
\text { predators }\end{array}$ \\
\hline
\end{tabular}




\subsubsection{Experimental set-up and trials}

I collected F. lapillum settlers from artificial substrates (Amman 2004) that were deployed within the harbour as part of another ongoing experiment (see Morton and Shima 2013 for details on substrate configuration). Collectors were cleared of new settlers at approximately 10 day intervals, and therefore, fish used in the experiment are assumed to be $<2$ weeks old (post-settlement age). Newly settled $F$. lapillum were placed in temporary holding tanks supplied with flow-through sea water (at the Victoria University Coastal Ecology Lab, VUCEL) for an acclimation period of 24-72 $\mathrm{h}$ before their introduction to experimental trials (described below). Over the entire experimental period, 596 settlers were collected $(25.8 \mathrm{~mm} \pm 2.3$, mean total length \pm standard deviation). Both adult F. lapillum $(61 \mathrm{~mm} \pm 5.3)$ and $F$. varium $(117 \mathrm{~mm}$ \pm 7.3 ) were collected locally around the Wellington region using dip nets and were starved for a 48 hour period prior to their use in trials.

Experimental trials were conducted in large, circular outdoor tanks with a bottom surface area of $0.9 \mathrm{~m}^{2}$. The bottom of the tank was covered with a $3 \mathrm{~cm}$ deep layer of locally obtained beach sand and a single layer of $\sim 30$ cobbles was haphazardly arrayed to provide natural refuge (Fig. 2.1B). Two individual stipes of the common macroalgal species Cystophora retroflexa, briefly rinsed in a fresh water solution to remove any potential food items from the stipe and cut to a standardized length of $20 \mathrm{~cm}$, were added to the tank for additional cover. As macroalgal identity did not have any significant effects on juvenile densities in surveys, only $C$. retroflexa was chosen for use in the experimental set-up, as previous evidence suggests settlers may prefer this algal type (McDermott and Shima 2006). For the full duration of experimental trials, tanks were supplied with a constant rate of flow-through seawater at approximately $10 \mathrm{~L}$ minute ${ }^{-1}$.

For each trial, predator species ( $F$. varium and $F$. lapillum) were added to the treatment tanks and allowed to acclimate for a $10 \mathrm{~h}$ period without food. At $1900 \mathrm{~h}$ on the day of the trials, predators were isolated in a section of the tank using a temporary mesh barrier (which allowed settlers to sense predator presence before being directly exposed to them) and 12 settlers of $F$. lapillum (randomly selected from the pool) were released into the predator-free area and allowed to acclimate for $1 \mathrm{~h}$. Prior to being released into the tank, settlers were individually measured by taking a photograph of the settlers next to a scale bar. The total length of each settler was recorded prior to being added to the tank. Settlers were added in the evening because reef fish 
commonly settle at dusk (Robertson et al. 1988) or at night (Victor 1986; Sweatman and St John 1990; Schmitt and Holbrook 1999), and this is also when most predator-induced mortality is thought to occur (e.g., Holbrook and Schmitt 2002). After the acclimation period, the predator barrier was removed, giving predators full access to recently settled F. lapillum for a period of 24 h. At $1700 \mathrm{~h}$ on the following day, all surviving $F$. lapillum settlers were removed and counted. All fish (settlers, adult $F$. lapillum, and $F$. varium) were used in only a single trial to ensure statistical independence. Trials were repeated over 8 different time blocks during the recruitment season of 2011-2012 to achieve replication (7 March, 9 March, 16 March, 23 March, 2 April, 4 April, 12 April, 16 April), and all 6 treatments were represented in each time block. Assignment of treatments to tanks was randomised in each time block to avoid any systematic bias caused by the experimental tanks.

\subsubsection{Statistical analysis for experimental trials}

I used mortality as the response variable for statistical analysis and calculated expected mortality in the multi-species treatments (for both the additive and substitutive designs) by subtracting the expected survival from the total number of prey (12). I examined mortality between treatments by using a GLMM fitted with a binomial distribution (number of prey alive, number of prey dead) and used a post-hoc Tukey's test to assess differences among treatments. A one-way ANOVA indicated there were no differences in settler size between treatments $\left(\mathrm{F}_{(5,35)}=0.753, \mathrm{p}=0.526\right)$; however, there was a significant difference between the average size of settlers used in experimental tanks between the replicates (ANOVA, $F_{(7,35)}=2.442, p=0.038$ ), potentially caused by differences in collection dates or seasonal differences in settler size. In order to account for the potential effects of size on mortality, I included the average length of settlers for each tank as a covariate in the analysis. In order to test for emergent multiple predator effects in the multi-species treatment, I made comparisons between observed mortality and expected mortality (substitutive and additive) using a one-way ANOVA. 


\subsubsection{Statistical software}

All statistical analyses were completed using the statistical software R (R Core Team 2013). I used the package "Ime4" (Bates et al. 2013) with the function glmer to conduct all GLMMs and the package "multcomp" (Hothorn et al. 2008) for all post-hoc Tukey's tests. Likelihood ratio tests were conducted using the anova function in the "Ime4" package. For the relationship between juvenile and adult density, candidate models were generated from global models using the dredge function in the "MuMIn" package (Barton 2013). Model averaging was completed with the function modavg and predictions from model averaged parameter estimates were made with predict.modavg function, both in the "MuMIn" package in R. 


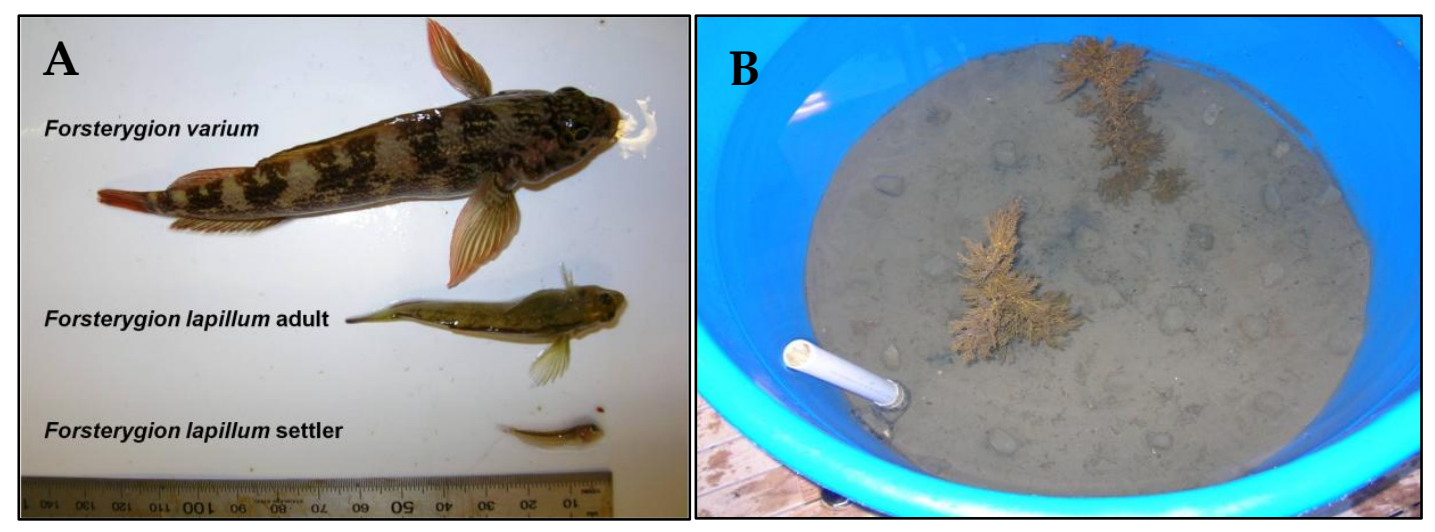

Figure 2.1 A The two fish species used in experimental trails: adult Forsterygion varium (predator species) and the adults and settlers ( 10 days past settlement) of Forsterygion lapillum. Scale bar at the bottom of the photograph is in millimetres. B Experimental trial tanks $\left(0.9 \mathrm{~m}^{2}\right.$ bottom surface area) with a sandy bottom, two stipes of Cystophora retroflexa and a light covering of cobbles to provide cover. The white stand-pipe used for excurrent flow is shown on the left side of the tank 


\subsection{Results}

2.4.1 Density patterns among sites, algal species, and sampling periods

In the surveys of juvenile and adult $F$. lapillum, I counted a total 1489 individuals (707 juveniles, 782 adults) and found that spatial overlap between adults and juveniles was high, with $92 \%, 86 \%$, and $81 \%$ of quadrats containing both adults and juveniles for sample periods 1,2 , and 3 , respectively. Densities varied significantly among sites for both juveniles $\left(X^{2}=21.889, p<\right.$ $0.001)$ and adults $\left(X^{2}=21.456, d f=1, p<0.001\right)$, although variation in densities among sites was more pronounced for juveniles (Fig. 2.2). Sampling period had no significant effect on juvenile density $\left(\mathrm{X}^{2}=21.889, \mathrm{df}=2, \mathrm{p}=0.898\right)$; however, adult density did vary with sampling date $\left(X^{2}=10.571, \mathrm{df}=2, \mathrm{p}=0.005\right)$. A post-hoc test revealed that adult densities during the second sampling period ( 4.17 adults $\mathrm{m}^{-2} \pm 0.23$, mean $\pm \mathrm{SE}$ ) were significantly higher (Wald test $\mathrm{Z}=-3.216 \mathrm{p}=0.003)$ than the third sampling period $\left(3.14\right.$ adults $\left.\mathrm{m}^{-2} \pm 0.21\right)$, but did not differ significantly from the first sampling period $\left(3.56\right.$ adults $\mathrm{m}^{-2} \pm 0.26$, Wald test $\mathrm{Z}=1.864, \mathrm{p}=$ 0.149). In addition, adult density was significantly higher in the monospecific stands of $C$. maschalocarpum than in the mixed stands $\left(\mathrm{X}^{2}=12.085, \mathrm{df}=1, \mathrm{p}<0.001\right.$, Fig. 2.3), but juvenile densities were unaffected by algal identity $\left(X^{2}=0.0004\right.$, df $=1, p=0.984$, Fig. 2.3). 

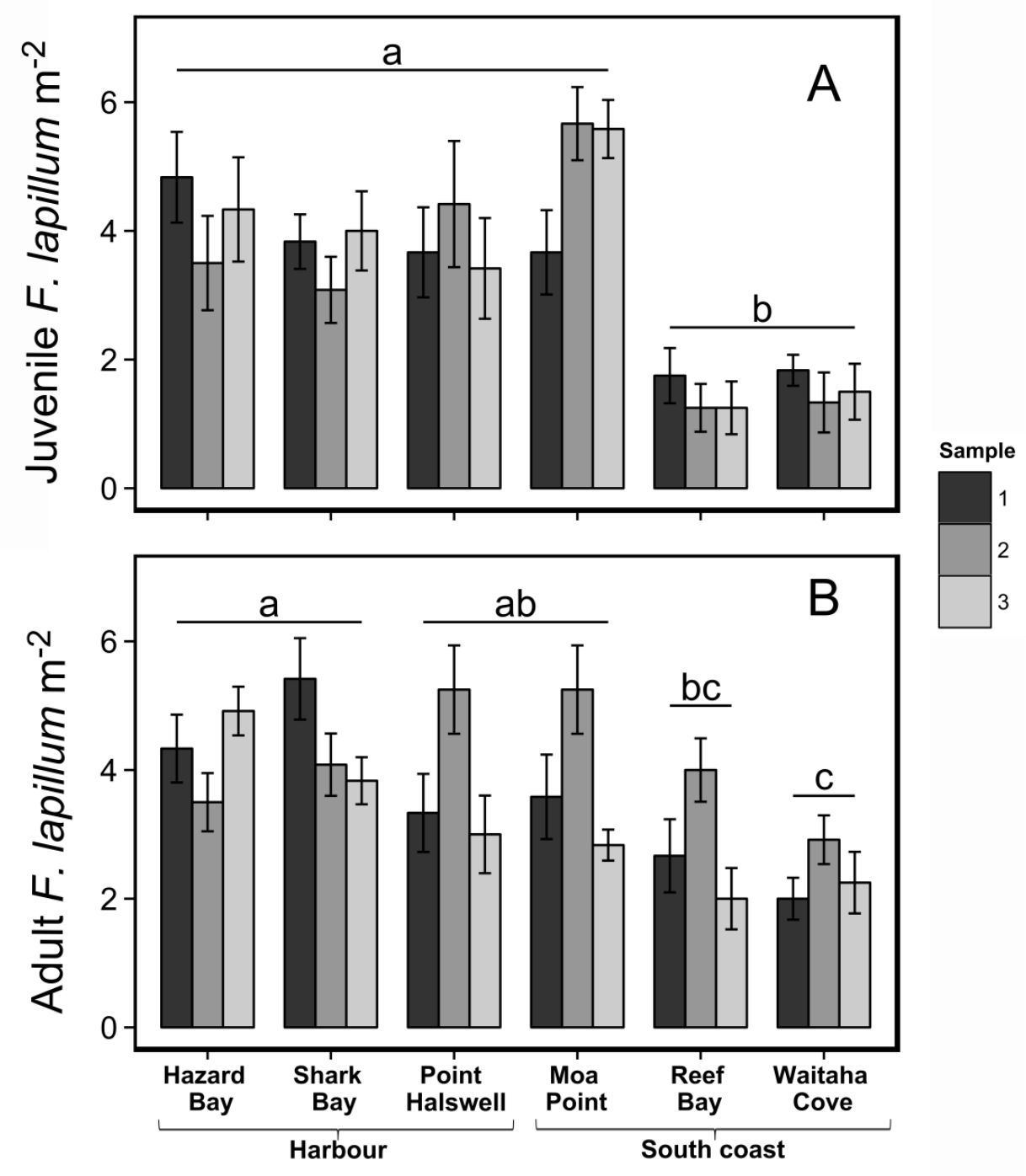

Figure 2.2 Densities of juvenile (panel A) and adult (panel B) F. lapillum (mean \pm SE) at six sites within two locations around the Miramar peninsula (Harbour: Hazard Bay, Shark Bay, Point Halswell; exposed southern coast: Moa Point, Reef bay Waitaha Cove) over three sampling periods (harbour: 9 Feb, 18 Feb, 9 March; exposed southern coast: 10 Feb, 20 Feb, March 3). Sites with different lowercase letters represent significantly $(\mathrm{p}<0.05)$ different site means based on a post-hoc Tukey's test from a generalized linear mixed model with site as a fixed factor and a random factor of quadrat ID (sampling dates pooled) 

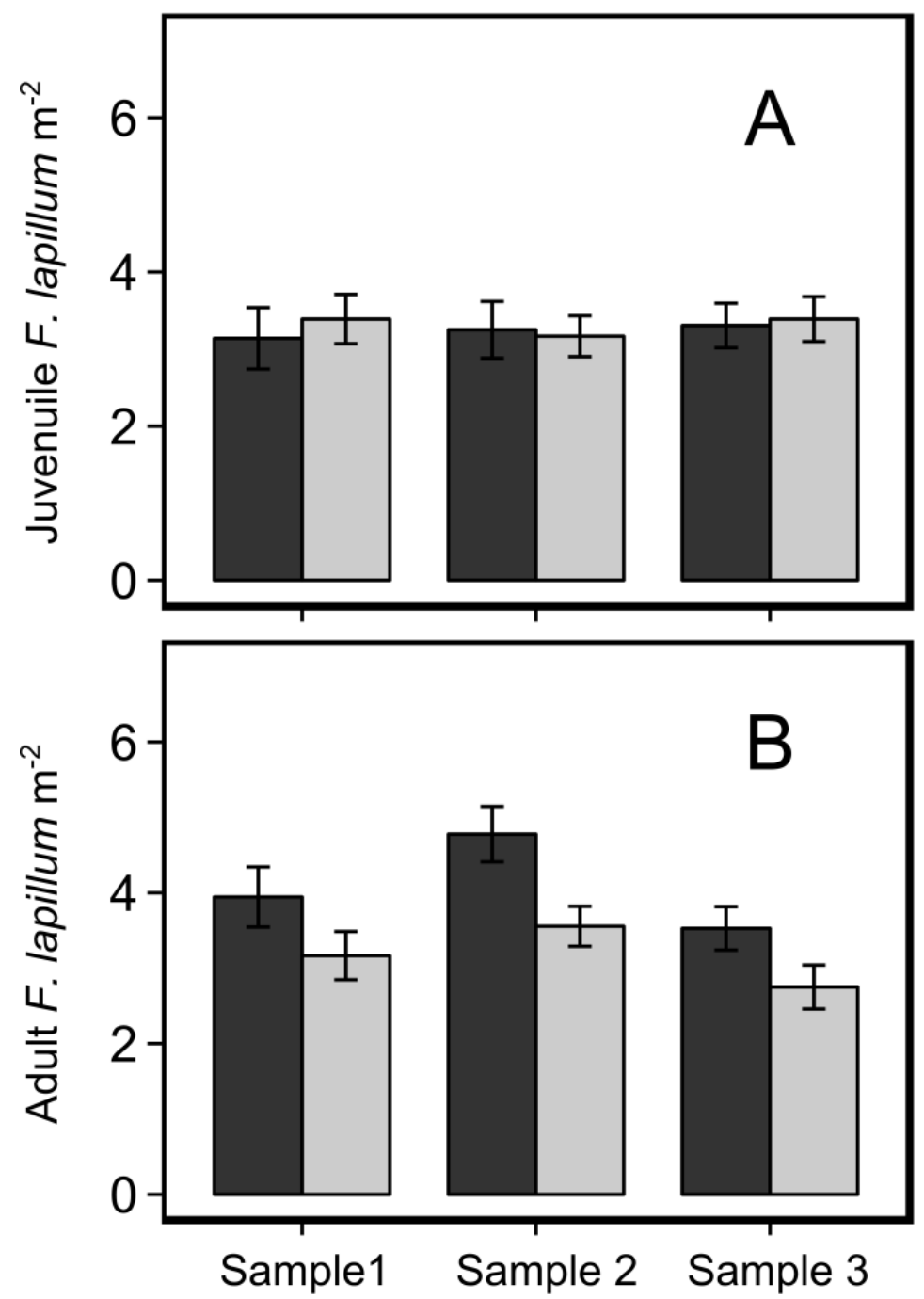

Figure 2.3 Densities of juveniles (panel A) and adults (panel B) in quadrats of Carpophyllum maschalocarpum (black bars) and mixed quadrats containing 50\% Carpophyllum maschalocarpum and 50\% Cystophora retroflexa (grey bars) over three sampling periods (harbour: 9 Feb, 18 Feb, 9 March; exposed southern coast: 10 Feb, 20 Feb, March 3). Results are pooled by location. Errors bars represent the mean \pm standard error 


\subsubsection{Relationship between juvenile and adult densities}

The number of models selected for model averaging based on the cut-off ( $\triangle$ AICc $<2$ ) was 6 for the full model. Adult density (including second-order adult density) was highly important, being present in all of the selected models. Second-order adult density was significant and negative (Table 2.3), indicating that the relationship between adult and juvenile density was positively curvilinear (e.g., a dome shaped curve). However, the relationship between adult density and juvenile density (i.e., the shape of the curve) varied significantly with sampling period (Table 2.3). Macroalgal identity and location did not have any significant effects on juvenile density or the effect of adult density on juvenile density (Table 2.3).

When sampling periods were analyzed individually, the second-order term of adult density was significant for each sampling period (Table 2.4), suggesting that the relationship was positively curvilinear (Fig. 2.4). The number of models included in model averaging was 2 for the first sample period, 4 for the second sample period, and 3 for the third sample period. During the first sampling period, recruitment location had a significant effect on juvenile density (Table $2.4)$, with the south coast having lower juvenile densities $\left(2.42\right.$ juveniles $\mathrm{m}^{-2} \pm 0.30$, mean $\left.\pm \mathrm{SE}\right)$ than the harbour (4.11 juveniles $\left.\mathrm{m}^{-2} \pm 0.36\right)$. 


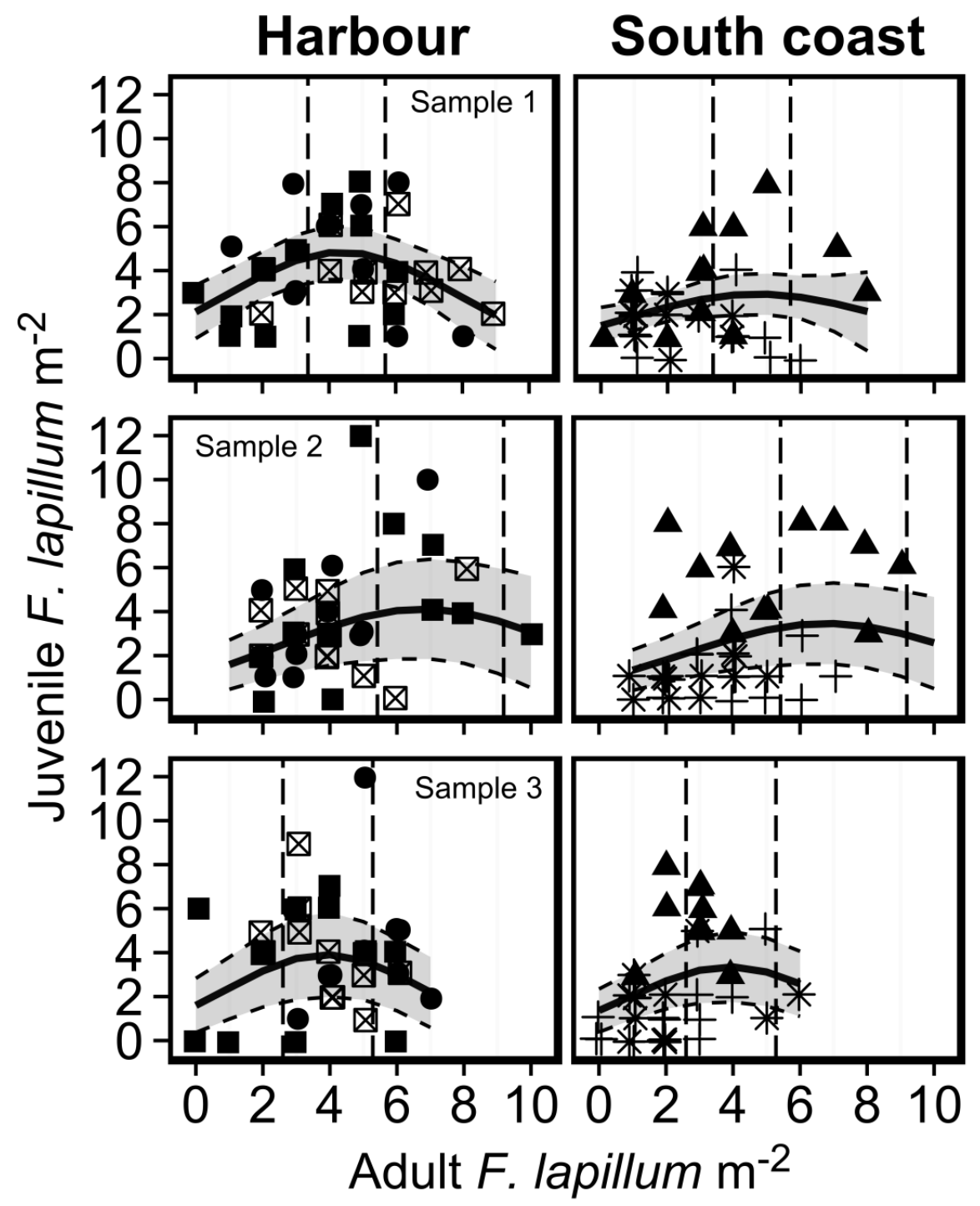

Figure 2.4 Predicted juvenile densities (bold line) for the quadratic model, split by sampling period (harbour: 9 Feb, 18 Feb, 9 March; exposed southern coast: 10 Feb, 20 Feb, March 3) and location. Plots are given for visualisation purposes and do not accurately reflect the variation that is accounted for the by the random effect of site in the full statistical model. As there were no significant differences between macroalgal species, predictions shown are based on Carpophyllum habitats only to ease interpretation. Dashed lines around the model fit represent confidence intervals $(2 \mathrm{x}$ unconditional standard errors) for the predicted model fit. Points represent a single quadrat density $\left(n=36\right.$ panel $\left.^{-1}\right)$, shifted by 0.1 axis increments to facilitate visualisation of overlapping points. Sites are marked by different shape patterns: Harbour Hazard Bay (@), Shark Bay (凶), Point Halswell (ם); South coast - Reef Bay (+), Moa Point $(\Delta)$, Waitaha Cove $(*)$. Vertical dashed lines represent $95 \%$ confidence intervals on the peak value of the quadratic curve 
Table 2.3 Standardised (input variables scaled to a mean of 0) model-averaged regression estimates for combined model (that included sampling period as fixed factor). Unconditional standard errors (SE) include model selection uncertainly in their estimates. Upper and lower confidence intervals $(\mathrm{CI})$ are given on the regression estimates. Relative importance (RI) gives the sum of the AICc weight for each model that parameter was included in (a RI of 1 indicates that parameter was present in all of the averaged models). For the interaction between adult density and sampling period, individual regression estimates are given for each level of sampling period (e.g., Sample 2, Sample 3), but in all cases the models included interactions between all levels of sampling period. $\mathrm{P}$-values are based on Wald test $\mathrm{Z}$ scores with significant values given in bold

\begin{tabular}{|c|c|c|c|c|c|c|c|}
\hline Parameter & Estimate & $\mathbf{R I}$ & SE & $\begin{array}{c}\text { Lower } \\
\text { CI }\end{array}$ & $\begin{array}{c}\text { Upper } \\
\text { CI }\end{array}$ & $\begin{array}{c}\mathrm{z} \\
\text { value }\end{array}$ & p-value \\
\hline (Intercept) & 1.18 & & 0.18 & 0.83 & 1.54 & & \\
\hline Polynomial adults & -0.50 & 1.00 & 0.16 & -0.82 & -0.18 & 3.07 & 0.002 \\
\hline Adult density & 0.17 & 1.00 & 0.15 & -0.13 & 0.47 & 1.08 & 0.267 \\
\hline Sample 2 & -0.19 & 1.00 & 0.10 & -0.39 & 0.01 & 1.83 & 0.067 \\
\hline Sample 3 & -0.01 & 1.00 & 0.09 & -0.20 & 0.17 & 0.14 & 0.892 \\
\hline $\begin{array}{l}\text { Adult density x } \\
\text { Sample } 2\end{array}$ & 0.71 & 1.00 & 0.23 & 0.26 & 1.16 & 3.12 & 0.002 \\
\hline $\begin{array}{l}\text { Adult density } \times \\
\text { Sample } 3\end{array}$ & -0.02 & 1.00 & 0.23 & -0.48 & 0.44 & 0.061 & 0.945 \\
\hline Location & 0.25 & 0.61 & 0.16 & -0.06 & 0.55 & 1.53 & 0.117 \\
\hline Macrolgal identity & 0.03 & 0.22 & 0.05 & -0.06 & 0.12 & 0.64 & 0.520 \\
\hline $\begin{array}{l}\text { Polynomial adults } \times \\
\text { location }\end{array}$ & 0.11 & 0.13 . & 0.12 & -0.13 & 0.34 & 0.87 & 0.384 \\
\hline Adults $\times$ location & 0.07 & 0.11 & 0.09 & -0.11 & 0.25 & 0.74 & 0.462 \\
\hline
\end{tabular}


Table 2.4 Standardised (input variables scaled to a mean of 0) model-averaged regression estimates for each sampling point separately. The number of models averaged for each sampling was 2, 4, 3 respectively. Unconditional standard errors (SE) include model selection uncertainly in their estimates. Upper and lower confidence intervals (CI) are given on the regression estimates. Relative importance (RI) gives the sum of the AICc weight for each model that parameter was included in (a RI of 1 indicates that parameter was present in all of the averaged models). For the interaction between adult density and sampling period, individual regression estimates are given for each level of sampling period (e.g., Sample 2, Sample 3), but in all cases the models included interactions between all levels of sampling period. P-values are based on Wald test $\mathrm{Z}$ scores with significant values given in bold

\begin{tabular}{|c|c|c|c|c|c|c|c|c|}
\hline Sample & Parameter & Estimate & SE & Lower CI & Upper CI & RI & $\begin{array}{c}\mathrm{z} \\
\text { value }\end{array}$ & $\begin{array}{c}\text { p- } \\
\text { value }\end{array}$ \\
\hline \multirow[t]{5}{*}{1} & (Intercept) & 1.30 & 0.10 & 1.09 & 1.50 & & & \\
\hline & Poly adult density & -0.61 & 0.24 & -1.09 & -0.13 & 1.00 & 2.49 & 0.013 \\
\hline & Adult density & 0.27 & 0.16 & -0.04 & 0.58 & 1.00 & 1.72 & 0.086 \\
\hline & Location & 0.26 & 0.10 & 0.06 & 0.45 & 1.00 & 2.56 & 0.010 \\
\hline & $\begin{array}{l}\text { Poly adult density X } \\
\text { Location }\end{array}$ & -0.28 & 0.24 & -0.76 & 0.20 & 0.36 & 1.14 & 0.255 \\
\hline \multirow[t]{4}{*}{2} & (Intercept) & 1.04 & 0.20 & 0.64 & 1.43 & & & \\
\hline & Poly adult density & -0.47 & 0.24 & -0.93 & -0.01 & 1.00 & 1.99 & 0.047 \\
\hline & Adult density & 0.73 & 0.24 & 0.26 & 1.20 & 1.00 & 3.04 & 0.002 \\
\hline & Location & 0.23 & 0.19 & -0.14 & 0.60 & 0.38 & 1.21 & 0.733 \\
\hline \multirow[t]{5}{*}{3} & (Intercept) & 1.35 & 0.24 & 0.88 & 1.82 & & & \\
\hline & Poly adult density & -0.98 & 0.39 & -1.74 & -0.22 & 1.00 & 2.52 & 0.012 \\
\hline & Adult density & 0.13 & 0.21 & -0.27 & 0.53 & 1.00 & 0.63 & 0.526 \\
\hline & Location & -0.45 & 0.34 & -1.12 & 0.22 & 0.34 & 1.32 & 0.187 \\
\hline & Macroalgal identity & 0.09 & 0.13 & -0.17 & 0.35 & 0.19 & 0.70 & 0.482 \\
\hline
\end{tabular}




\subsubsection{Experimental test of intercohort multiple-predator effects and cannibalism}

Mortality was consistently higher in the tanks containing $F$. varium (either 1 or 2 individuals) compared with the control tank $(F$. varium - Wald test $\mathrm{Z}=-3.26$, p value $=0.001 ; 2$ $F$. varium - Wald test $\mathrm{Z}=-4.21$, $\mathrm{p}$ value $<0.001$ ). Juvenile $F$. lapillum experienced an average mortality rate of $22 \% \pm 6 \% \mathrm{SE}(2.6 \pm 0.7$ individuals, mean $\pm \mathrm{SE})$ over $24 \mathrm{hr}$ when $1 F$. varium was present, and $45 \% \pm 9 \%(5.3 \pm 1.0$ individuals $)$ over $24 \mathrm{hr}$ when $2 \mathrm{~F}$. varium were present (Fig 2.5A). Background mortality (Control) was extremely low, with only one settler dying in the control tank during the entire experimental period. Mortality attributable to adult $F$. lapillum was low for both $F$. lapillum $(6 \% \pm 3 \%, 0.8 \pm 0.3$ individuals) and $2 F$. lapillum $(5 \% \pm 3 \%$, $0.6 \pm 0.3$ individuals) and did not differ from background mortality, although this was marginally non-significant $(F$. lapillum- Wald test $\mathrm{Z}=-1.75, \mathrm{p}=0.08 ; 2$ F. lapillum- Wald test $\mathrm{Z}=-1.656, \mathrm{p}=$ 0.09, Fig. 2.5A). However, there was a significant effect of average settler length on the probability of settler survival (Wald test $\mathrm{Z}=2.50, \mathrm{p}=0.012$ ). The mean average settler length in experimental tanks was $25.9 \mathrm{~mm} \pm 0.11$ standard deviation and ranged from $23.8 \mathrm{~mm}$ to 28.4 mm. I tested the effects of length on survival in each of the six treatments separately with a generalized linear model (binomial distribution) and found that average settler length was positively correlated with survival in both the $2 \mathrm{~F}$. lapillum treatment $(\beta=1.87$, Wald test $\mathrm{Z}=$ 2.41, $\mathrm{p}=0.016$ ) and the multispecies treatment $(F$. lapillum and $F$. varium, Wald test $\mathrm{Z}=2.52$, $\beta=0.817, \mathrm{p}=0.012$ ). Observed survival in the multi-species treatment ( $F$. lapillum and $F$. varium) was not significantly different from expected mortality when tested under either the additive $\left(\right.$ ANOVA, $F_{(1,14)}=0.224 \mathrm{p}=0.64$, Fig. $\left.2.5 \mathrm{~B}\right)$ or substitutive designs $\left(\right.$ ANOVA, $\mathrm{F}_{(1,14)}=$ $0.430, \mathrm{p}=0.52$, Fig. 2.5B). 


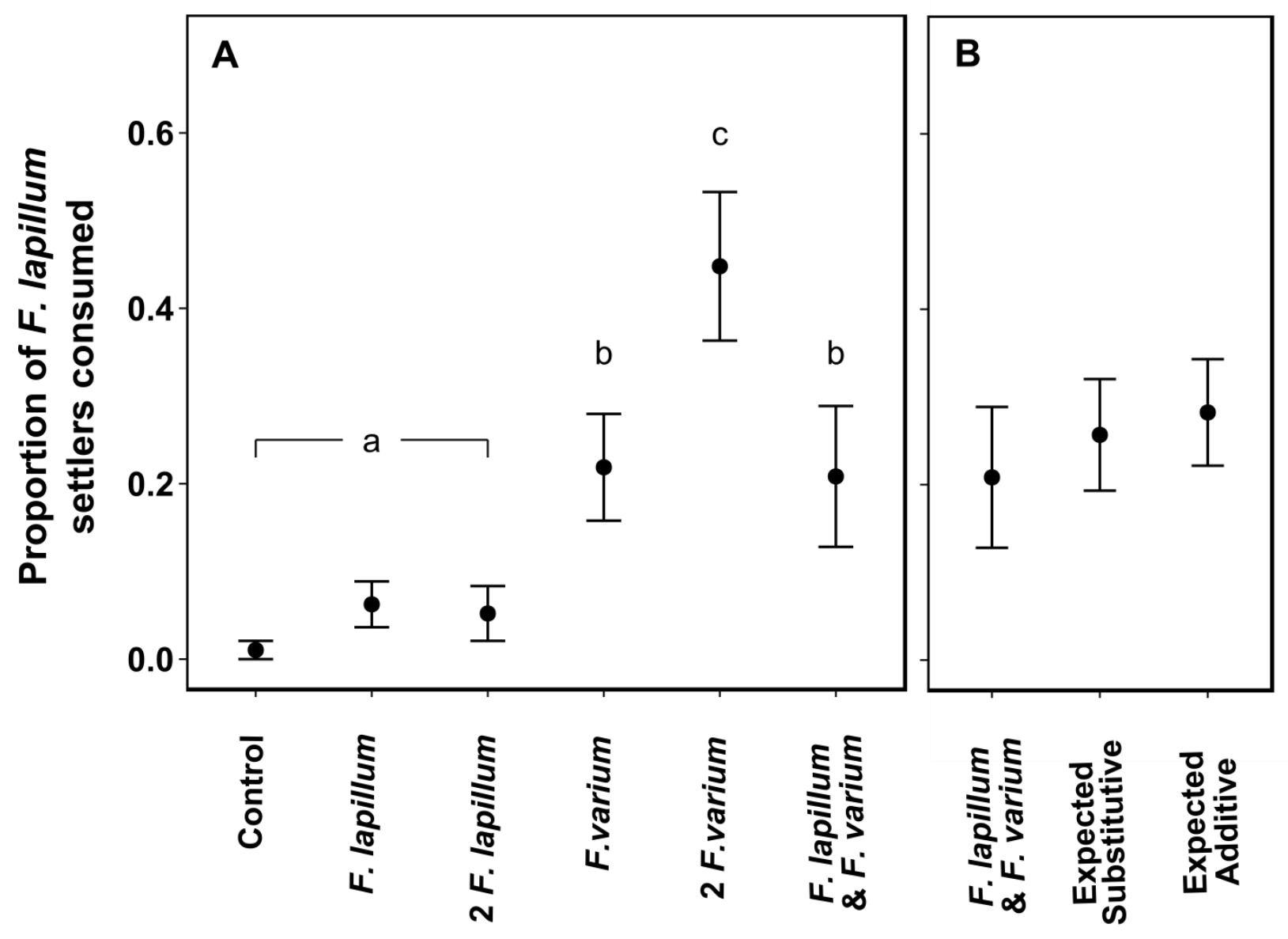

Figure 2.5 A Mean proportion \pm SE of F. lapillum settlers consumed over a 24 hour period ( $\mathrm{n}=8$ trials) across the 6 experimental treatments. Letters represent statistically significant groups based on a post hoc test $(\alpha=0.05)$ from a generalized linear model with a binomial family. B Comparisons between mean proportion $\pm \mathrm{SE}$ of $F$. lapillum settlers consumed over a 24 hour period in the multi-species treatment and expected proportion $\pm \mathrm{SE}$ of settlers consumed determined from additive and substitutive calculations. There was no significant difference between observed mortality in the multi-species treatment and the expected mortality based on either the substitutive or additive designs, indicating that emergent mortality effects were not observed 


\subsection{Discussion}

The effects of resident adults on the spatial variation in juvenile recruitment may range from positive (Hunt et al. 2011) to negative (Webster 2004; Samhouri et al. 2009). In my study, the juvenile-adult density relationship followed a dome-shaped curve, which suggests that a combination of factors that both increase and decrease recruitment near resident adults may interact to drive variation in recruitment strength (e.g., Vermeij 2005, Adam 2011, Wilson and Oseberg 2002). The presence of a negative correlation in the juvenile-adult density relationship is consistent with (i) patterns of negative density dependence or (ii) changes in habitat selection by fish during settlement (assuming larval fish selectively chose habitats) to avoid high densities of adults (e.g, Ayllón et al. 2013). Negative density dependence between adults and juveniles at higher adult densities may be caused by increased rates of opportunistic cannibalism (Smith and Reay 1991), increased interspecific predation in the presence of adults due to competition for predator free refuges (Samhouri et al. 2009), or a combination of both. The curvature pattern was consistent between algal species; although, adult $F$. lapillum density was lower in mixed habitats. Furthermore, this pattern appeared to be unaffected by recruitment location, suggesting that intercohort interactions may be consistent throughout the regional population. It should be noted however, that unmeasured substrate variables (i.e., the cover of rocks, gravel, etc.) may have affected my ability to detect significant differences between algal habitats and locations. This field study suggests that intercohort interactions in F. lapillum may be limiting recruitment at intermediate to high adult densities. With the size classes used in the survey design, I was unable to account for the importance of other post-settlement processes, outside of intercohort interactions (e.g. intracohort density dependence), that may have influenced the relationship between juvenile and adult density. Consequently, the observed negative correlation may be a combination of several post-settlement processes. However, if the negative correlation observed in $F$. lapillum is the result of increased settler mortality at high adult densities (likely via interspecific predation), intercohort effects may cause cyclical inter-annual variation in recruitment (Borgstem et al. 1993; Bjørnstad et al. 1999; Webster 2003).

In order to investigate the potential mechanisms by which adults may negatively affect

survival of recently settled fish, I used a laboratory experiment to evaluate the effects of both cannibalism and multiple predators on settler survival. In the heterospecific predator treatments, 
high mortality was consistently observed in the F. varium tanks, and given the co-occurrence of juvenile F. lapillum and F. varium in the field (Perez-Matus and Shima 2010), this indicates that F. varium could be an important predator on F. lapillum settlers. Cannibalism occurred in 50\% of the F. lapillum trials and $38 \%$ of the trials containing $2 F$. lapillum; however, mortality rates were marginally non-significantly different from the control tank due to a high degree of variability among replicates within these treatments. Differences in average settler size per tank (at the start of each trial) may have influenced the results, as a post hoc analysis suggested that tanks with larger individuals appeared to have higher rates of survival. From this, I infer that cannibalism by adult $F$. lapillum may be present, but perhaps limited to the smallest individuals (e.g., Foster et al. 1988), potentially due to reduced prey vulnerability as prey size increases (Bailey and Houde 1989), or decreased predator success due faster prey evasion (Mitchell et al. 2011). The jaw structure in F. lapillum has evolved for small prey items, and the diet of $F$. lapillum rarely contains larger prey (Feary et al. 2009). Therefore, it is possible that adults may only be able to consume settlers of a relatively small size. Samhouri et al. (2009), found that size selective mortality on small settlers increased with adult density in the goldspot goby (Gnatholepis thompsoni), resulting in larger settlers at high adult densities, although the authors focus on competition and not cannibalism as a potential cause. However, if cannibalism is size limited in F. lapillum, it may help explain variation in selective mortality of settlers seen in other studies (Shima and Swearer 2009b). Evidence was lacking for emergent effects (competition/predator facilitation or predator interference); however, my ability to detect emergent effects, given a potential size effect on mortality and relatively low rates of cannibalism, may have been limited. The experimental trials used relatively low adult densities compared with what was observed in the field, and at natural field densities of adults, cannibalism may be a major contributing factor to settler mortality.

The presence of positive as well as negative intercohort processes may have complex effects on recruitment dynamics, and especially on the importance of habitat quality in mediating recruitment variation (Adam 2011). Post-settlement movement between habitats appears to be rare in F. lapillum (Smith 2010; Shima et al. 2012), indicating that the post-settlement distribution of settlers may be maintained throughout demersal life, and suggests that resident 
adult density and habitat quality may reliably co-vary in space. Positive spatial co-variation in adult and juvenile density may occur if larval fish use conspecific cues as an indirect measure of habitat quality (Stamps and Krishnan 2005), or if they selectively chose high quality habitats where adults are prevalent (Wellington 1992). Alternatively, if F. lapillum settlement is random, and survival probabilities within habitat patches are consistent from year-to-year, adult and juvenile density may co-vary based on the effects of habitat quality on survival, rather than any direct cues from adults (e.g, Wilson and Osenberg 2002). Recently settled F. lapillum may have the ability to select particular habitats at settlement (McDermott and Shima 2006); however, the influence of conspecifics on habitat selection is currently unknown. The presence of both positive and negative relationships between adults and juveniles may potentially obscure patterns of density dependence across heterogeneous habitats (e.g., cryptic density dependence, Shima and Osenberg 2003), and highlights the importance of examining intercohort relationships when investigating how density dependence regulates populations.

The second sampling period showed significant temporal variability in the local maxima (the curve peak), with the peak in juvenile density occurring at higher adult densities. The temporal variability observed here may have been caused by a variety of unmeasured factors, such as temporal variation in larval supply or the timing of settlement pulses. For instance, $F$. lapillum may settle in discrete pulses at roughly monthly intervals (Smith and Shima 2011), and if I sampled immediately after a settlement pulse, there may not have been sufficient time for negative effects of adults to take place (e.g., the competitive exclusion of juveniles into new habitats). I can only speculate on the role of larval supply in causing the observed temporal variation; however, the temporal variability observed here indicates that short-time scale processes may significantly alter intercohort density relationships and mortality-driven abundance patterns, and/or the ability to detect them.

My field results are supported by the findings of Wellenreuther (2007) from a more northern site in New Zealand, who also found that the relationship between juvenile density and adult density was initially positive in $F$. lapillum, with a negative correlation as adult densities increased. Differences between the studies highlight the generality of this observed pattern across both large and small spatial scales. For instance, Wellenreuther (2007) made comparisons of new settler densities $(<30 \mathrm{~mm})$ in $16 \mathrm{~m}^{2}$ random quadrats encompassing several habitat types, 
whereas I sampled juveniles $(<40 \mathrm{~mm})$ in $1 \mathrm{~m}^{2}$ quadrats within well-known settlement habitats. Furthermore, Wellenreuther (2007) found that the relationship between settler density and adult density peaked at approximately 1-1.5 adults $\mathrm{m}^{-2}$ for 3 different triplefin species including $F$. lapillum (based on graphical inference rather than statistical analysis), whereas I found higher and temporally variable peak values here. Densities of both juveniles and adults in this study were 3-4 fold higher, possibly because I targeted specific habitats where F. lapillum is known to be most abundant (Feary and Clements 2006; Wellenreuther and Clements 2008) or due to the different sampling unit sizes between studies $\left(16 \mathrm{~m}^{2}\right.$ vs. $\left.1 \mathrm{~m}^{2}\right)$. Nonetheless, despite the differences in survey methods, habitat types, fish densities, and geographical location (Wellington vs. Auckland), both studies found that juvenile densities appear to positively correlate with adult densities initially to a peak, followed by a negative decline as adult density increases, indicating that this negative relationship appears to be a consistent trend in F. lapillum.

Our study highlights the potential role that previous cohorts may play in shaping recruitment patterns and suggests that adult density may have a non-linear effect on recruitment strength. The initially positive relationship between juveniles and adults indicates that adults may facilitate recruitment of juveniles; a rigorous test of this might include experiments to evaluate the ability of larvae to detect and respond to spatial variation in adult density (e.g., preferentially settle sites with increased densities of adults, Forrester 1999). At higher adult densities, the negative correlation between juveniles and adults suggests a form of density dependence that has the potential to regulate population dynamics. This study contributes to the growing number of studies that have identified negative interactions between adults and juveniles in several species of small-bodied reef fish including: other triplefin species (Wellenreuther 2007), gobies (Forrester 1995; Wilson 2005; Samhouri et al. 2009), and basslets (Webster 2004). Given the common occurrence of these negative interactions between adults and juveniles in small-bodied reef fish, an important functional group on both temperate and tropical reefs, intercohort interactions could have important bottom-up effects for entire reef ecosystems. Investigating the relative importance of intercohort interactions in comparison with density independent (e.g., larval supply) or intracohort density dependent processes will be important in understanding recruitment dynamics at the population level. 
Chapter 3 - Application of intercirculus
spacing to measure body growth in the
common triplefin 


\subsection{Abstract}

Somatic growth is an important metric for the description of fish populations (e.g., dynamics, performance, 'health'), but is often difficult to measure directly. I measured the efficacy of using intercirculus spacing on fish scales to estimate somatic growth rates (i.e., changes in body length) of fish. First, I collected sexually mature fish from multiple populations near Wellington, New Zealand and tested relationships between body size and scale size. The body-scale size relationship was strongly positive and was unaffected by gender; however, I observed significant differences between certain collections sites, possibly caused by growth effects. Second, I monitored somatic growth rates and scale growth rates of tagged fish (in individuals originating from two different groups) in the laboratory to investigate how intercirculus spacing varied with somatic growth rate. Growth rates were monitored for 20 days on ad libitum diets and at the completion of the monitoring period new scale growth, circuli number, and average intercirculus spacing were measured. New scale growth and circuli number were both positively correlated with somatic growth. Average intercirculus spacing was also positively correlated with somatic growth rate, but this appeared to be affected by group age, with the older group showing a weaker relationship. Results suggest that intercirculus spacing may be an effective measure for estimating growth rates, although it may be limited to younger/smaller fish. 


\subsection{Introduction}

For fish biologists, somatic growth rates are important to quantify because they can indicate habitat quality (Meng et al. 2000; Amara et al. 2007) and mediate reproductive output (Reynolds and Gross 1992). Growth increments found on fish scales (circuli) are commonly used to reconstruct individual growth histories either by (i) measuring growth between yearly check marks (annuli) formed by seasonal decreases in the distance between circuli (Lee 1920; Alvord 1954), or by (ii) measuring growth between individual circuli (intercirculus spacing, daily to monthly timescales, Doyle et al. 1987). Intercirculus spacing can provide sub-annual growth estimates with a high temporal resolution (Hogan and Friedland 2010; Newhard et al. 2012; Marco-Rius et al. 2013); however, the use of intercirculus spacing has been limited to a small number of important fisheries species (Fisher and Percy 1990, 2005; Friedland 2000; Wells 2003), and this application is virtually unexplored in reef fish (although see Cheung et al. 2007).

In order to use intercirculus spacing to estimate growth, key relationships between somatic growth and scale growth need to be validated. First, the assumption that body size and scale size are proportionally related must be confirmed (Francis 1990). The correlation between somatic growth and growth in calcified structures may become weak or decoupled over time if growth rates influence the proportional relationship between somatic growth and scale growth (e.g., slower growing fish have larger otoliths than faster growing fish, Wells et al. 2003). Furthermore, differences in growth rates between populations or sexes may cause the bodystructure relationship to vary (Hoxmeier et al. 2001; Munday and Hodges 2004), which may affect comparisons of scale-derived growth rates between individuals. Second, the rate of circuli deposition (i.e. the temporal resolution of each growth measurement) must be determined and a positive correlation between somatic growth rate and intercirculus spacing must be verified. Validating the temporal patterns of circuli deposition is particularly important, because unlike otolith increments, circuli deposition is rarely daily and deposition rates may vary substantially between species (Cheung et al. 2007). In addition, the relationship between growth rates and intercirculus spacing may be obscured by changes in circuli deposition rates caused by age (Kingsford and Atkinson 1994) or growth rates themselves (Cheung et al. 2007; Peyronnet et al. 2007). 
Here, I investigate how intercirculus spacing may be used to estimate previous growth in a temperate reef fish, the common triplefin (Forsterygion lapillum). The common triplefin is a small species of the Triptygeriidae (max total length $70 \mathrm{~mm}$ ) and is one of the most common benthic fish species in shallow coastal areas around New Zealand (Willis 2001; Wellenreuther et al. 2007a; Wellenreuther et al. 2008). Otolith microstructure has been used extensively to examine growth during early life history and has revealed a variety of growth-related traits that have impacts on survival (Shima and Swearer 2010; Smith and Shima 2011); however, there is currently no effective sub-lethal measure of juvenile or adult growth. In the first part of this study, I quantify how the relationship between body size and scale size varies among fish sampled from multiple populations and between sexes. Secondly, I monitor somatic growth rates in fish across size classes under controlled conditions to (i) characterise the relationship between growth rate and intercirculus spacing and (ii) estimate the rate of circuli deposition.

\subsection{Methods}

\subsubsection{Validation of length-body scale relationship}

To estimate the relationship between total fish length and scale size, I sampled scales from fish collected around the Wellington region of New Zealand. Between 31 May and 19 June 2011, I led dive teams (using SCUBA) that haphazardly collected (i.e., without any formal method of randomization) 100 sexually mature $F$. lapillum from four different collection sites: Kaiwharawhara ( $\mathrm{n}=28$ individuals) and Shark Bay ( $\mathrm{n}=25$ individuals), located within the enclosed harbour; and Moa Point ( $\mathrm{n}=24$ individuals) and Waitaha Cove ( $\mathrm{n}=23$ individuals), located on the exposed south coast. Sites were non-randomly selected to ensure sampling was done over a broad spatial scale. I focused on fish $>40 \mathrm{~mm}$ in length because (i) this is approximately when sexual organs appear (facilitating gender identification) and (ii) I hypothesized that accumulated growth effects (i.e., a decoupling of somatic growth from scale growth) would be more common and easier to detect in older fish. Divers with hand nets captured fish from a broad area across each site to obtain a representative sample. All fish were collected from shallow water $(<5 \mathrm{~m}$ deep) on rocky reef habitats. Fish were transported back to the Victoria University Coastal Ecology Laboratory (VUCEL) in buckets and euthanized in an ice and sea water slurry. For each fish, I measured total length (TL) and sex (determined by 
dissection). Fish were then individually stored and frozen until a sample of scales was removed for subsequent analysis.

\subsubsection{Scale removal and measurement}

As with many perciform fish, the majority of F. lapillum scales are ctenoid (with a "toothed" posterior end) and are found covering most of the body (Jawad 2005). During the life of a fish, lost scales are naturally replaced; however, these regenerated scales are visibly deformed and contain no growth information prior to scale loss. I chose to only measure non-regenerated scales to ensure that scale size was the result of growth occurring from scale formation, and was unbiased by regeneration. I collected 2 non-regenerated scales from the first two scale rows below the lateral line, directly posterior to the pectoral fin (Fig. 3.1A). Scales from this region can be easier to read in some species (Takahashi 2008), and in triplefins, scales from this area share a similar squamation pattern (size and growth shape of scales, Jawad 2005). Removed scales were lightly cleaned in distilled water to remove tissue and then allowed to dry on a microscope slide. I added a small drop of immersion oil to increase clarity of the circuli under magnification and placed a cover slip on top of each sample. Scales were photographed at 50X magnification using an Olympus® digital camera attached to a compound microscope. I then measured the total length of both scales from the anterior margin to the posterior end (Fig. 3.1B) using the image analysis software Image Pro ${ }^{\circledR}$ v5.0. 

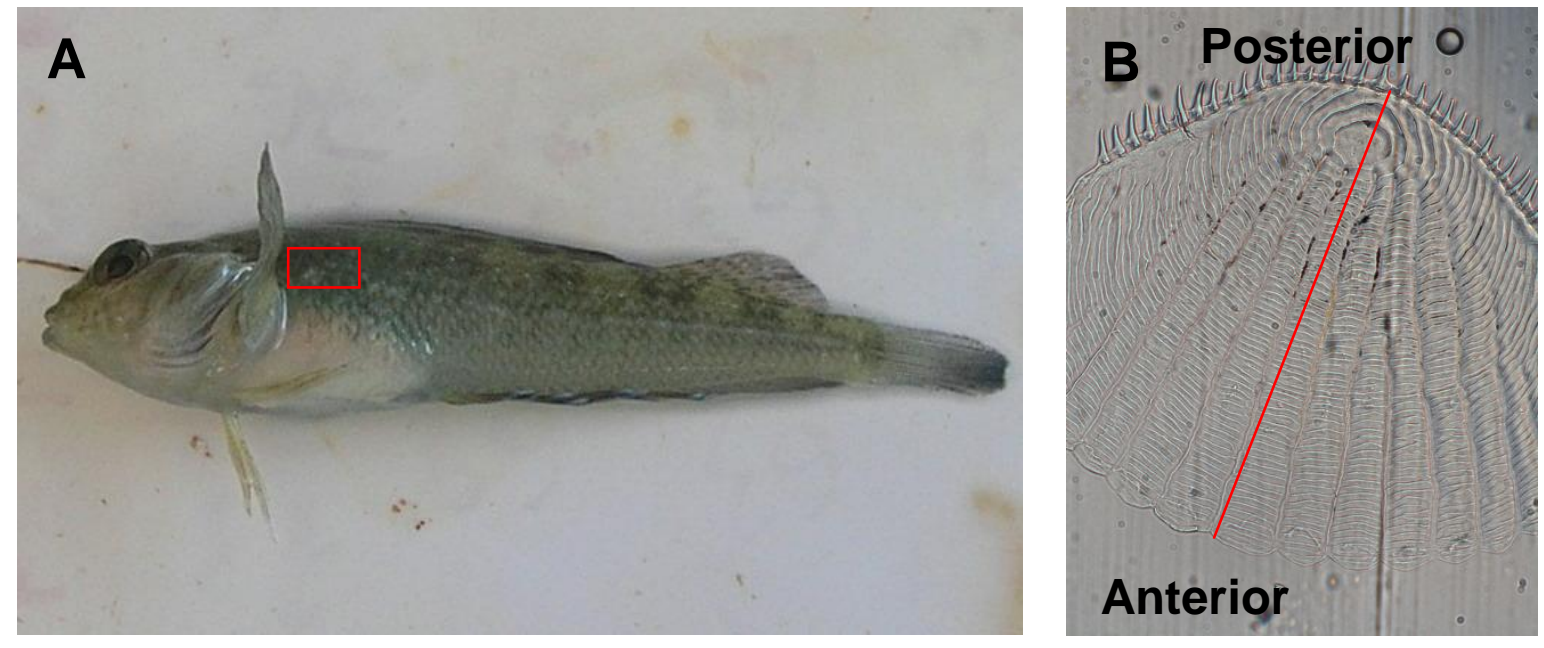

Figure 3.1 A An individual $F$. lapillum with the area of scale removal highlighted B A photograph of a removed scale under 50X magnification showing the line of scale size measurement in red. 


\subsubsection{Statistical analysis of body- scale length relationship}

In order to measure the relationship between total length and scale size, and how this was affected by collection site and gender, I ran a series of linear regression models. To create a dataset, I randomly selected one scale (of the 2 scales I had removed from each fish and prepared on each microscope slide) and measured the length of that scale for analysis. Specifically, I was interested in determining if the total length-scale relationship varied between genders or collection sites; therefore, I analyzed the influence of gender and collection site separately. For gender, I created two separate models: the first model contained fixed factors of scale length and gender and the second model included the same factors plus a two-way interaction (scale length $\times$ gender) to test whether the total length-scale relationship was consistent between sexes. In order to determine if gender influenced the relationship, I compared models with and without the interaction term using a likelihood ratio test. Likewise, I created two separate models for collection site: the first model contained fixed factors of scale length and collection site and the second model included the same fixed factors plus a two-way interaction (scale length $\times$ collection site) to determine if the relationship between total length and scale length was affected by collection site. Again, I compared the models with and without the interaction term using a likelihood ratio test. If interaction terms were significant between gender and scale length or site and scale length, it would indicate that predictions of total length from scale length would significantly vary by collection site/gender.

In a separate analysis, I examined how much the relationship between body size and scale size differed depending on which scale was measured (i.e., would estimates of total length vary widely based upon which scale was removed from the fish). However, as results from the previous analysis indicated gender did not interact with scale length, I only conducted this analysis with a fixed factor of collection site. As before, I created a data set by randomly selecting one of the two available scales from each fish and then using scale length from that scale. I then repeated this selection procedure 1000 times to create 1000 datasets which were composed of unique sets of randomly drawn scales (1 from each fish). In order to determine how variable regression coefficients and $\mathrm{R}^{2}$ values of the linear models were across the datasets, I ran the same analysis as before: one model included only scale length as a fixed factor and the other included scale length and collection site with an interaction term (scale length $\times$ collection 
site). In addition, I also ran linear models (with scale length as a fixed factor) for each collection site separately (4 models total) to understand how sensitive regression coefficients from individual collections sites were to scale selection. Overall, in order to quantify the variability in the regression coefficients and the $\mathrm{R}^{2}$ value caused by the different scale selections across each of the 1000 data sets, I calculated the mean and standard deviation of both the regression coefficients and the $\mathrm{R}^{2}$ value. Furthermore, for the model with all collection sites included, I recorded whether or not the interaction term between collection site and scale length was significant (i.e., did the statistical test suggest that the relationship between total length and scale length differed significantly between sites).

\subsubsection{Growth monitoring}

To determine the correlations between growth rate, intercirculus spacing and circuli deposition I collected 50 fish across a range of size classes (26mm-59 mm TL) and monitored their growth in the laboratory. I collected fish from a single population at Kau Point (41 17' 19" S, 174 50'00" E) on 11 Feb 2014 and transported all fish to VUCEL. I chose to sample fish from a single population in order minimize the potential effects of among-population variability in body-length scale relationships and maximise statistical power. Upon arrival at VUCEL, I allowed fish to acclimate for 5 days to laboratory conditions and feeding regimes. During acclimation period and the trials, I fed fish daily ad libitum on a mixed diet of frozen arrow squid (Nototodarus sloanii) and high protein fish food (New Life Spectrum ${ }^{\circledR}$ Grow Formula $0.5 \mathrm{~mm}$ pellets). On 16 February 2014, I submerged fish in a calcein dye solution, to mark scales and indicate scale size at the beginning of the monitoring period. In order to enhance osmotic uptake of the dye, I placed each fish in seawater with an artificially high salt content (55 ppt) for 10 minutes prior to submersion in the dye solution (reverse induction method). After 10 minutes had elapsed, fish were rinsed thoroughly in fresh seawater for 30 seconds, and then placed in the dye solution $(1000 \mathrm{mg} / \mathrm{L})$ for 3 hours. At the end of the staining period, I washed all fish thoroughly in fresh seawater and placed them in a recovery tank for a period of 24 hours before introduction into the tanks. At the end of the 24 hour recovery period on $17 \mathrm{Feb}$, I used an Olympus digital camera (stabilized on a tripod approximately $10 \mathrm{~cm}$ above the fish), to photograph each individual lying flat on its lateral side next to a scale bar. I then measured the 
length of each fish from the snout to the tail using the photograph and image analysis software Image $\mathbf{J}^{\circledR}$. Based on initial fish length, I ordered fish according to total length and assigned fish to one of ten glass aquaria in a way that maximized length differences between individuals within tanks, but also reduced the potential occurrence of cannibalism by grouping smaller settlers with smaller adults. This was done to facilitate the identification of individuals across tanks without the need for invasive tagging procedures. Tanks were supplied with flow-through seawater at a rate of $1 \mathrm{~L}$ minute ${ }^{-1}$ for the duration of the trial.

During the trial, all fish were fed ad libitum three times daily for a 20 day period. Water temperature throughout the monitoring period was measured with a TidBit data logger. Average temperature during the trial was $10.78^{\circ} \mathrm{C} \pm 1.08$ (mean $\pm \mathrm{SD}$ ). At the end of the 20 day period, I removed scales from the same area as described previously (see section 3.3.2 Scale removal and measurement) and measured the total length of all fish using the same photographic set-up as the beginning of the experiment. Scales were mounted on a glass side in a drop of immersion oil and photographed at $100 \mathrm{X}$ magnification under a fluorescent bulb (to indicate the calcein mark) using a compound scope. Using the image analysis software Image Pro ${ }^{\circledR}$ v5.0, I took scale measurements from one point in the middle of the anterior end of the scale (at the scale margin along the straight axis between the posterior and anterior end of the scale). I took three measurements from the one area of the scale margin: (i) the amount of growth on the scale from the calcein mark to the edge of the scale, and (ii) the intercirculus spacing in the region of new growth, and (iii) the number of new circuli formed from the calcein mark to the scale margin.

\subsubsection{Statistical analysis of growth monitoring trials}

The distribution of total lengths among the fish collected for the trials appeared to be strongly bimodal, and a Hartigan's dip test for uni-modality (Hartigan and Hartigan 1985) indicated that the distribution was indeed multi-modal $(\mathrm{D}=0.088$, $\mathrm{p}$-value $=0.003)$. This strongly suggests that I sampled individuals from two distinct size groups. I partitioned individuals into groups based on the distribution of total lengths (TL) using a cut-off point of $45 \mathrm{~mm}$ (Fig. 2.2). This resulted in two groups: group 1 (mean TL=54.5 mm, sd=5.23) and group 2 (mean TL=34.1 $\mathrm{mm}, \mathrm{sd}=4.43$ ). I suggest that based on size differences, these two groups are likely year classes (group 1- recruitment in 2012-2013, group 2- recruitment in 2013-2014). 
I constructed four linear models to examine (1) the relationship between total growth (final TL-initial TL) and total scale growth, (2) the relationship between total growth and circuli deposition, (3) the relationship between growth rate (final TL-initial TL/20 days) and total length, and (4) the relationship between somatic growth rate and intercirculus spacing. For models 1, 2, and 4 I also included a fixed factor of group with an interaction term between group and the main fixed factor (e.g. model 1 - total scale growth $\times$ group) to determine if the relationships with scale traits varied between groups. I employed a model selection process using AICc values to rank three possible factor combinations (main factor, main factor with group, and main factor, group, interaction with group). A cut-off of $\triangle \mathrm{AICc}<2$ was used as a criterion for a top model set (Burnham and Anderson 2002).

For model 1 (total scale growth) I did a secondary analysis testing each group separately (regardless of the significance of the interaction term) to test for potential growth effects (i.e., a decoupling of somatic growth and scale growth at lower growth rates) caused by larger size or age. I hypothesized that scale growth for group 1 (older, larger and slower growing fish) would describe less variation in total growth (i.e., have a lower coefficient of determination , $\mathrm{R}^{2}$ ) than scale growth in group 2 (younger, faster growing fish). In order to test this hypothesis, I performed a non-parametric bootstrap with replacement to estimate confidence intervals,(simulations $=1000$ ) on the $\mathrm{R}^{2}$ values for each group to make inferences about statistical significance. The bootstrap distribution was heavily skewed; therefore, I constructed confidence intervals using the bias corrected and accelerated method (Efron 1987).

For model 4, results indicated that groups differed in the relationship between somatic growth and intercirculus spacing, so I analyzed the two groups separately to determine how much variation in growth rate within each group was explained by average intercirculus spacing. Again, I performed a non-parametric bootstrap with replacement to estimate confidence intervals on the $\mathrm{R}^{2}$ values for each group and make inferences about statistical significance. As before, the bootstrap distribution was heavily skewed; therefore, I constructed confidence intervals using the bias corrected and accelerated method (Efron 1987). 


\subsubsection{Statistical Software}

All statistical analysis was conducted in R (R Core Team 2013). For the Hartigan's test I used the 'diptest' package. I used the $1 \mathrm{~m}$ function in the "base" package to test linear relationships between factors. Likelihood ratio tests were conducted using the anova function in the "base" package. Bootstrapping was conducted using the boot.ci function in the "boot" package (Canty and Ripley 2013). 


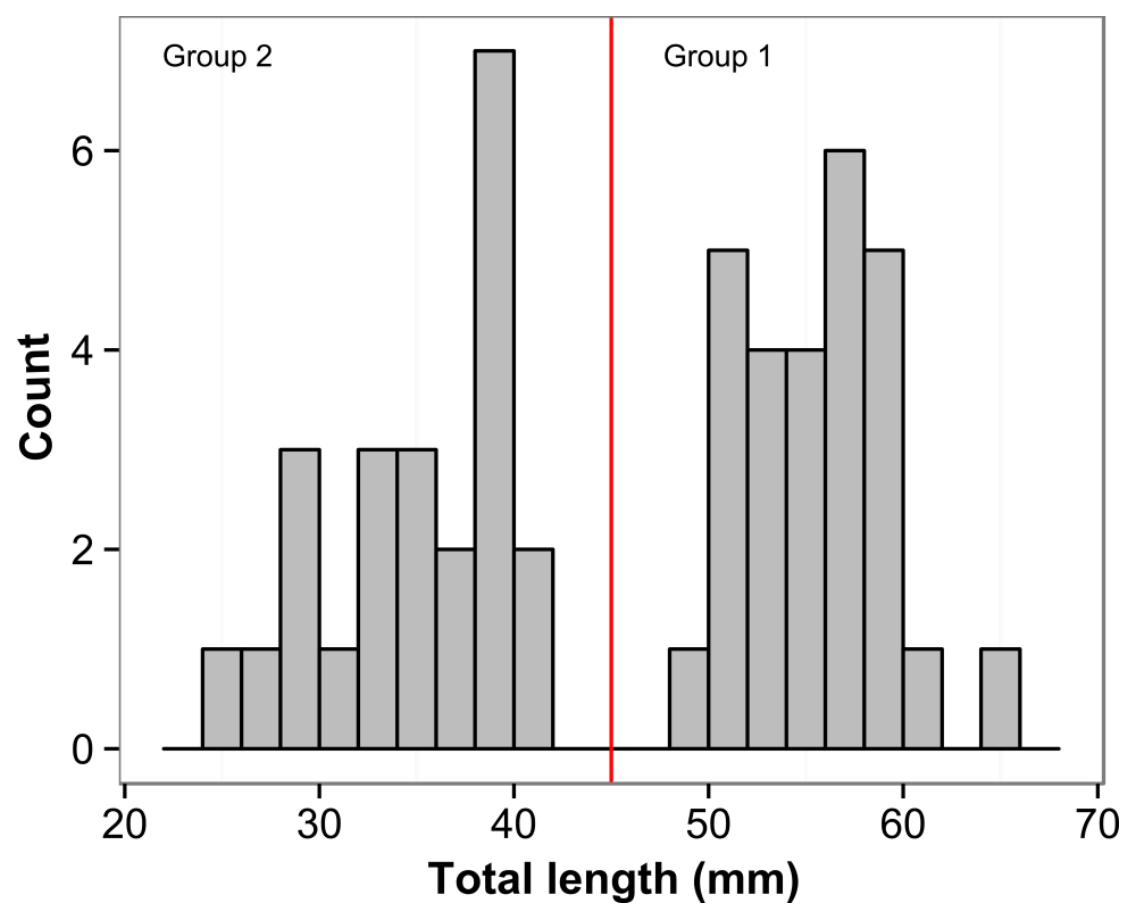

Figure 3.2 Histogram of total length in fish collected for laboratory monitoring of growth rate. The red line represents the selected cut-off point separating the first and second groups. Bin width is $2 \mathrm{~mm}$. 


\subsection{Results}

\subsubsection{Body-scale relationship}

For the gender model, there was no significant interaction between gender and scale length (likelihood ratio test, $\mathrm{F}$ value $=0.733, \mathrm{p}=0.3941$ ). For collection site, models with and without the interaction (scale length $\times$ collection site) had very similar AICc values (With interaction $\mathrm{AICc}=475.4$, without interaction $\mathrm{AICc}=475.2$ ), but the likelihood ratio test suggested that the interaction term did not improve the model fit $(\mathrm{F}=2.302, \mathrm{p}=0.082)$. However, this was only marginally non-significant and in the sensitivity analysis of scale selection 399 of the 1000 different randomized scale selections (each random selection was a unique combination of selected scales) found that sites differed significantly in their body-scale relationship $(p<0.05)$. Therefore, I ran both a linear regression for sites pooled (1 model) and sites separately (4 models) to investigate the relationship between total length and scale length (Table 3.1, Fig. 3.3). Variation in regression coefficients for each site based on randomly selected scales is shown in Fig. 3.4. 
Table 3.1 Regression outputs $(\beta)$ from linear regression between total length and scale length for sites pooled and sites separated including confidence intervals on the regression estimate (CI), F values, degrees of freedom (df), $p$ values, and coefficient of determination $\left(R^{2}\right)$. Repeated analysis shows the mean regression coefficients \pm standard deviation and the mean $R^{2}$ values \pm standard deviation taken from 1000 datasets constructed by randomly selecting one of the two prepared scales for each collected fish.

\begin{tabular}{cccccccccc}
\hline & \multicolumn{4}{c}{ Randomly selected scale } & \multicolumn{2}{c}{ Repeated Analysis } \\
\cline { 2 - 10 } Model & $\boldsymbol{\beta}$ & CI & F & df & $\mathbf{p}$ & $\mathbf{R}^{\mathbf{2}}$ & \multicolumn{3}{c}{$\mathbf{R}^{\mathbf{2}}$} \\
\hline \hline Sites pooled & 0.026 & $0.022,0.029$ & 206.6 & 1,98 & $<0.001$ & 0.675 & $0.026 \pm 0.0005$ & $0.709 \pm 0.024$ \\
Kaiwharawhara (H) & 0.021 & $0.013,0.029$ & 28.8 & 1,26 & $<0.001$ & 0.5073 & $0.021 \pm 0.001$ & $0.524 \pm 0.069$ \\
Shark Bay (H) & 0.011 & $0.002,0.0200$ & 5.8 & 1,23 & 0.024 & 0.2016 & $0.013 \pm 0.002$ & $0.230 \pm 0.061$ \\
Moa Point (SC) & 0.028 & $0.018,0.037$ & 37.8 & 1,22 & $<0.001$ & 0.6315 & $0.0291 \pm 0.001$ & $0.709 \pm 0.050$ \\
Waitaha Cove (SC) & 0.021 & $0.016,0.030$ & 43.5 & 1,21 & $<0.001$ & 0.659 & $0.0215 \pm 0.001$ & $0.651 \pm 0.049$
\end{tabular}




\subsubsection{Growth monitoring}

Four fish had to be excluded from the statistical analysis because they escaped their tanks into the sea table during the trials (and their identities could not be reconciled). All other individuals remained within tanks; relative size differences among individuals persisted over the 20-day trial and facilitated individual measurements of somatic growth $(n=46)$. However, a calcein stain could not be detected in 3 of the remaining 46 fish; consequently, these fish were excluded from scale growth analyses $(n=43)$. AICc values indicated that top models had strong support for both the scale growth model (second best model $\Delta \mathrm{AICc}=2.34$ ) and the circuli number model (second best model $\Delta$ AICc $=2.34$ ). Total somatic growth was positively correlated with scale growth (Fig 3.5A) and was also significantly higher in group 2 (Table 3.2). When groups were analyzed separately, scale growth was positively correlated with somatic growth for both groups (group1 $-\beta=0.0303, \mathrm{t}=3.132, \mathrm{p}=0.005, \mathrm{R}^{2}=0.318$ [bootstrapped confidence intervals $=0.061,0.632]$, group $2-\beta=0.0403, \mathrm{t}=7.908, \mathrm{p}<0.001, \mathrm{R}^{2}=0.777$ [bootstrapped confidence intervals $=0.354,0.934]$ ). For the scale circuli model, total somatic growth was positively correlated with scale circuli number (Fig. 3.5B) and the number of new circuli was significantly higher in group 2 (Table 3.2).

Daily growth rate was negatively correlated with total length (Table 3.2, Fig. 3.5C). A total of 38 fish formed at least two circuli, which allowed for the measurement of intercirculus spacing. AICc values showed support for two separate models for intercirculus spacing (second model $\triangle \mathrm{AICc}=1.16$ ). The top model included fixed factors of average intercirculus spacing and group, while the second model included an interaction term between average intercirculus spacing and group. A likelihood ratio test indicated that the addition of the interaction term did not significantly improve the model fit $(\mathrm{F}=1.368, \mathrm{p}=0.250)$ and therefore, I used the top model (without the interaction term) to make inferences. Average intercirculus spacing had a significant and positive correlation with daily growth (Table 3.2), with daily growth significantly higher in the second group (Fig. 3.5D). When I separated the groups for analysis, average intercirculus spacing in group 1 had a significant correlation with daily growth $(\beta=0.00856, \mathrm{r}$ $=0.456$, intercept $=-0.0547, \mathrm{df}=1,17, \mathrm{p}=0.0497, \mathrm{R}^{2}=0.208$ [bootstrapped confidence intervals $=$ $0.001,0.526])$. For group 2 , average intercirculus spacing also had a significant correlation with 
daily growth $\left(\beta=0.0159, \mathrm{r}=0.634\right.$, intercept $=-0.0586, \mathrm{df}=1,17, \mathrm{t}=3.386, \mathrm{p}=0.004, \mathrm{R}^{2}=0.403$ [bootstrapped confidence intervals $=0.091,0.697]$ ). 
Table 3.2 Regression coefficients, t-values and p-values for 4 different models from the laboratory monitoring of somatic growth rates.

\begin{tabular}{|c|c|c|c|c|}
\hline Response variables & Fixed factors & $\boldsymbol{\beta}$ & t-value & p-value \\
\hline \multirow[t]{4}{*}{ Total somatic growth $(\mathrm{mm})$} & Scale growth & 0.0390 & 8.57 & $<<0.001$ \\
\hline & Group & 0.8837 & 3.14 & 0.003 \\
\hline & Circuli number & 0.3620 & 6.22 & $<0.001$ \\
\hline & Group & 0.0458 & 2.57 & 0.014 \\
\hline \multirow[t]{3}{*}{ Daily growth $\left(\mathrm{mm} \mathrm{day}^{-1}\right)$} & Total growth & -0.0050 & -7.23 & $<0.001$ \\
\hline & Average intercirculus spacing & 0.0125 & 4.002 & $<0.001$ \\
\hline & Group & -0.1180 & -8.939 & $<0.001$ \\
\hline
\end{tabular}




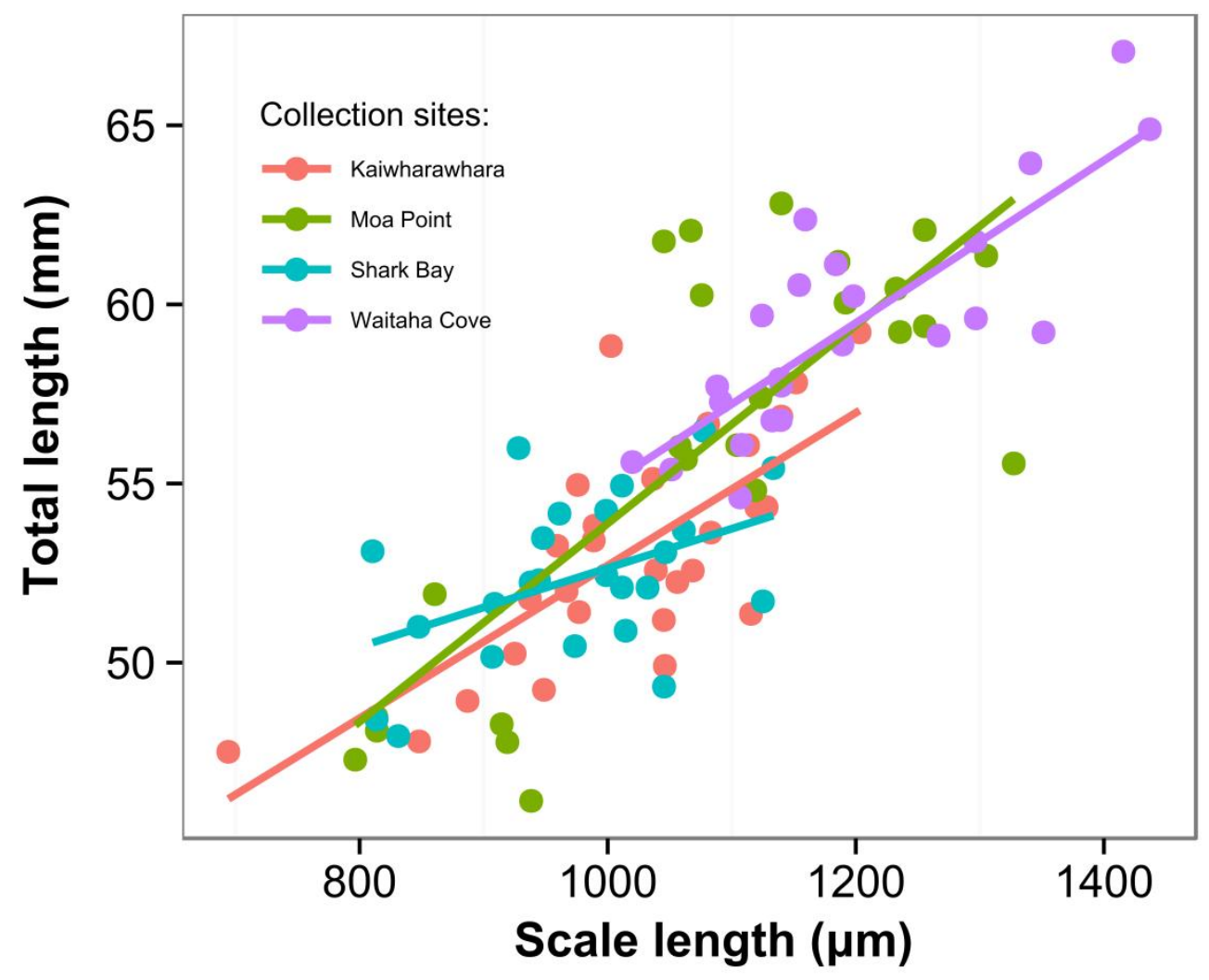

Figure 3.3 Regression fits between total length and scale length for four sites from the Wellington region. Regression fits are separated by site (indicated by colour). 


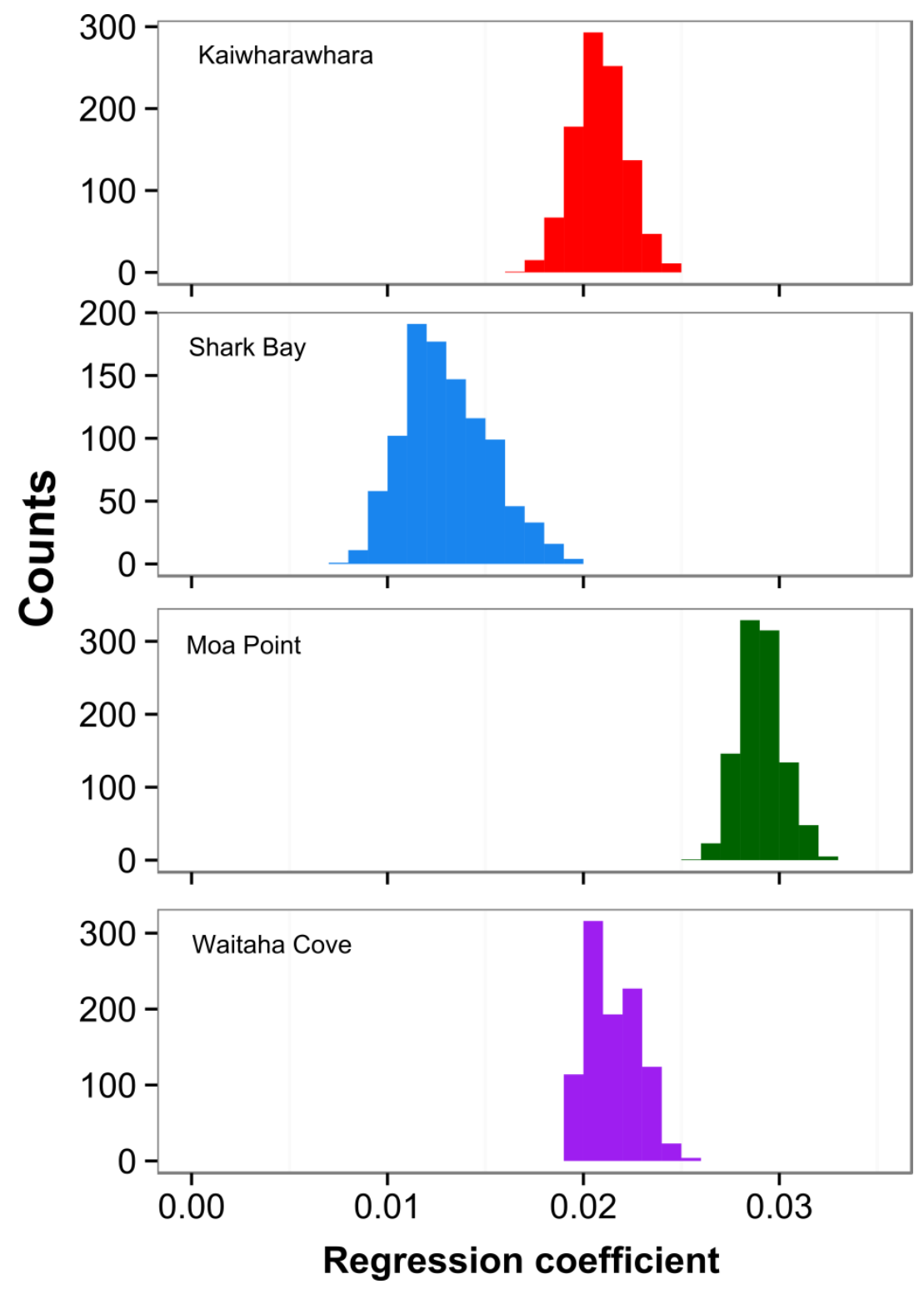

Figure 3.4 Histogram of regression estimates between total length and scale length for 1000 unique random scale selections (from one of two scales) separated by collection site. Each count represents one of the regression estimates taken from the 1000 data sets. 

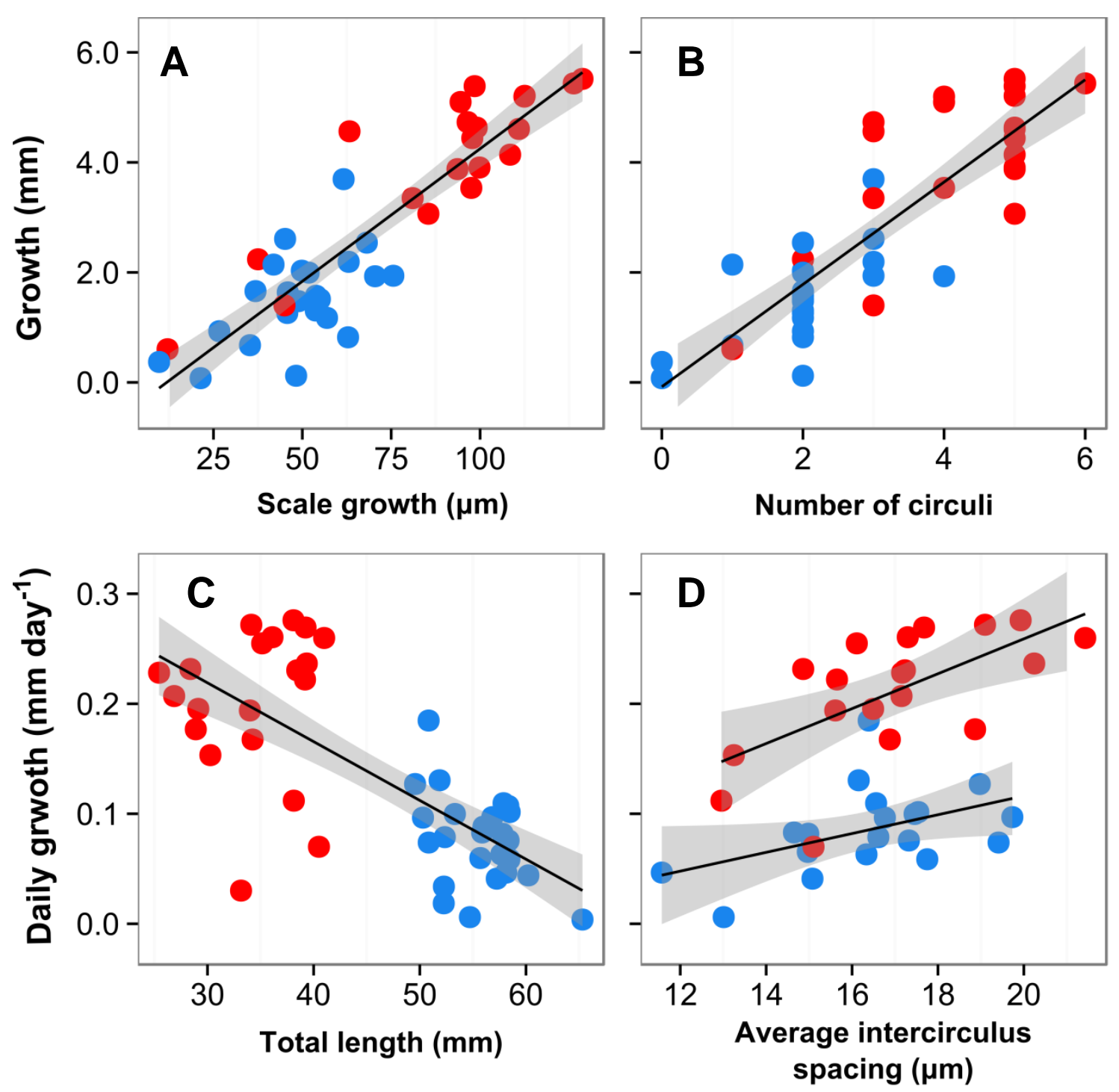

Figure 3.5 Relationships between somatic growth, scale growth, and total length $\mathbf{A}$ The relationship between total amount of somatic growth and the total amount of scale growth $(\mathrm{n}=$ 43) B The relationship total amount of somatic growth and the number of circuli formed on the new scale growth $(n=43)$ C The relationship between average daily growth rate and the total length of the fish at the beginning of the experiment $(n=46) \mathbf{D}$ The relationship between average daily growth rate and the average intercirculus spacing (regression lines done separately for each group, $n=38$ ). Groups are identified by colour (group 1- $\mathbf{0}$, group 2- $\mathbf{0}$ ). Regression lines represent a linear regression between the predictor and the response (except for panel $\mathrm{d}$, where regressions were done separately for each group). Grey confidence bands represent the confidence interval on the linear regression fit. 


\subsection{Discussion}

I measured the body-scale relationship from multiple populations and monitored growth rates in tagged individuals to evaluate the use of intercirculus spacing to estimate growth in the common triplefin. Intercirculus spacing was positively correlated with growth rate, despite circuli deposition also increasing positively with growth rate. Fisher and Percy (1990) observed the same pattern of covariance between intercirculus spacing, circuli deposition, and growth rate in salmon, suggesting scale characteristics may commonly respond to increased growth rate in this fashion. In my study, the strength of the relationship between growth rate and intercirculus spacing appeared to vary between groups. For the second (younger) group, the relationship was strongly positive, and the observed correlation coefficient was reasonably similar to those accepted as useful for determining growth from intercirculus spacing in other species $(r=0.65-$ 0.71 Doyle et al. 1987; r=0.36. Fukuwaka and Kaeriyama 1997). In comparison with the second group, the slope of the relationship in the first group (older) was reduced by a factor of about 2 and was only marginally significant. Although statistical analysis indicated that there were no significant differences in the slopes between groups (i.e. no interaction between group and intercirculus spacing), the power to detect this interaction may have been too low given my small sample size (post-hoc power $=0.2062$ ). Likewise, the variance explained by intercirculus spacing in group 1 was $\sim 50 \%$ less than group 2 , but again my ability to detect statistical differences between the $\mathrm{R}^{2}$ values using bootstrapped confidence intervals may have been limited by the small sample size. A previous study examining scale morphological characteristics in juvenile snapper (Pagrus auratus) from New Zealand also found that relationships between somatic growth and scale morphology may become decoupled in older fish (Kingsford and Atkinson 1994). This may be a result of slower growth rates, as some evidence suggests that scale characteristics may only be a reliable measure of growth if the growth rates are high (among species, Cheung et al. 2007). In my study, slower growth rates in adults (group 1) may have weakened the relationship between growth rate and intercirculus spacing. Overall, my results suggest that intercirculus spacing can be useful for monitoring juvenile growth, but its ability to track growth in larger fish may be limited.

For group 2, intercirculus spacing explained $40 \%$ of the variation in growth rates, which is comparable with Doyle et al. (1987) who found $\mathrm{R}^{2}$ values ranging from 0.11-0.50. $\mathrm{R}^{2}$ values 
in my study were less than those of Fisher and Percy (2005) who found relatively high $\mathrm{R}^{2}$ values (0.55-0.82) between circuli spacing and scale growth (i.e. they did not directly measure the correlation between intercirculus spacing and somatic growth rates). Variation in the growthintercirculus spacing relationship could have been introduced by several factors of the monitoring design. For instance, my study was limited in that I followed growth for a short period of time, only a limited number of full circuli had formed at the scale margin, and my sample size was low, given the variation in intercirculus spacing. Furthermore, I relied upon natural variation in growth on an ad libitum diet (similar to experiment 1, Doyle et al. 1987) and growth differences in the field (where resources may be limiting) may be more variable and better detected by intercirculus spacing. Alternatively, variation in intercirculus spacing could have also been by time-lags between somatic growth and intercirculus spacing (e.g., otoliths, Molony and Choat 1990). Given the amount of variation present, researchers should be cautious when attempting to estimate growth using intercirculus spacing.

In order to use intercirculus spacing across populations, patterns of body-size and scale relationships must be consistent. Scale size was a good predictor of body size overall, but appeared to vary between collection sites. In particular, Shark Bay appeared to have a shallower slope than the three other collection sites and also significantly higher variation in body-scale relationship. Shallower slopes in the relationship between body size and calcified structures can indicate the presence of growth effects, where the size of the calcified structure is larger than expected for a particular length (Campana 1990). Results from the laboratory monitoring indicate that the relationship between growth and scale growth (regression estimate and $\mathrm{R}^{2}$ ) was reduced in group 1 (i.e. shallower slope and less variation explained), which supports the hypothesis that growth rate effects may also be present in scales. Fish from Shark Bay were the smallest fish in the sample, and assuming that recruitment in the Wellington region occurs at a similar time among collection sites, this smaller size may have been caused by slower growth rates. Previous studies have also found that growth-scale relationships changed between locations (Lepomis macrochirus, Hoxmeier et al. 2001), and my results also highlight the importance of quantifying site-specific relationships among calcified structures before estimating growth rates across populations. 
Overall, this study demonstrates that intercirculus spacing in younger groups $(<45 \mathrm{~mm})$ can reasonably estimate somatic growth but, suggests that the use of intercirculus spacing to measure growth in adults may require further investigation. Due to the proportionality between circuli deposition and growth, circuli cannot be used to measure age-at-size; however, given the positive relationship between body size and scale length (for most populations), intercirculus spacing can be used to measure relative growth rates among individuals at particular sizes. Averaging over groups of circuli (i.e. over size ranges) in younger fish (e.g. averaged over 10 circuli - Richards and Esteves 1997), may be a conservative and cautious approach to using intercirculus spacing in order to dampen variation and avoid bias introduced by measurement error or potential time-lags. 
Chapter 4 - The effects of previous growth history on male reproductive success in the common triplefin, Forsterygion lapillum 


\subsection{Abstract}

In marine reef fish, growth rates are vitally important, and individuals that successfully recruit to adulthood may differ in their growth histories. Certain growth histories may be more reproductively successful than others; however, the effects of growth rates on variation in reproductive success among individuals has rarely been quantified. I measured the influence of juvenile growth histories (derived from fish scales) on male reproductive success (clutch and egg size) for the common triplefin (Forsterygion lapillum) during the 2011-2012 spawning season at two spawning sites in Wellington, New Zealand. Both egg number and egg size decreased over the spawning season. Early growth rate (determined from the first 10 scale circuli) was negatively correlated with clutch size, and was the only male trait significantly correlated with clutch size. Analysis of sampling dates separately indicated that this negative trend was driven primarily by a strong negative correlation during the second sampling period. Clutch size was highly variable, and early growth rate only explained a small amount of the total variation in clutch size $\left(\mathrm{R}^{2}=0.156\right)$. Results suggest that growth rates from earlier life stages may influence reproductive output, although the underlying mechanism is not currently understood. 


\subsection{Introduction}

In most mating systems, females invest more energy per offspring than males, and as a result, females typically select mates, preferentially mating with certain male phenotypes over others (Trivers 1972). Female choice usually leads to non-random variation in male mating success, with evidence suggesting that females preferentially choose males with traits or resources that increase offspring fitness via either their enhanced survival and/or subsequent reproductive success (Jennions and Petrie 1997). In polygamous mating systems, males compete for mating opportunities with multiple females. Males may compete directly with one another, for control of resources and/or access to females (intra-sexual selection), or indirectly via female selection (intersexual selection) (Emlen and Oring 1977). Mating success can vary substantially between individual males in a population (Clutton-Brock 1988), resulting in a disproportionate distribution of reproductive output among males. This, in turn, has implications for the genetic diversity of populations (Serbezov et al. 2010) via changes in effective population size (Hedgecock 1994), and may influence the evolutionary patterns of populations over time. Consequently, considerable effort has been directed at understanding the patterns and sources of variation in male reproductive success (Clutton-Brock 1988).

In marine reef fish, male size appears to be a particularly important trait in determining reproductive success (Thompson 1986; Oliveira et al. 1999). For example, size may affect competitive interactions between males for mating resources, such as oviposition sites, with competitively dominant individuals monopolizing the highest quality sites and obtaining the majority of mating opportunities (Hastings and Peterson 2010). In addition, females may prefer larger males because they provide better parental care (Côté and Hunte 1989). However, the importance of male size may vary both between species (Hastings and Peterson 2010) and within species during the breeding season (Oliveira et al. 1999), and substantial variation in male mating success may remain unexplained when solely considering traits measured at time of spawning (Kelly 2008). In organisms that have multiple life history stages, experiences from previous life history stages, such as growth rates, may influence phenotypic traits (e.g., size) in adults that are important in determining reproductive success (Gimenez 2006; Stamps 2006; Wilkin and Sheldon 2009). Due to the close inter-relationship between growth and size, the interaction between the two may help explain observed variability in reproductive success among 
males, although studies into the direct effects of growth rates on male reproductive success have been limited.

Growth rates may affect reproductive success in males directly via female selection or alternatively, growth rates may co-vary with traits that are influential in reproductive success. For instance, evidence suggests that females may select males with traits that increase the fitness of their offspring (Kokko et al. 2003), and if growth rates are significantly heritable between fathers and offspring, females may select fast growing males (via honest signals from other male traits), regardless of current size or age, due to the fact the fast growth rates may improve offspring survival (Reynolds and Gross 1992). Growth rates may also be positively correlated with local resource availability (Jones 1986), and females may choose individuals in high quality habitats to increase egg survival via higher quality parental care (Knapp and Kovach 1991) or to reduce filial cannibalism by parental males (Hoelzer 1992; Kvarnemo et al. 1998). There may also be trade-offs between somatic growth and reproductive effort (Arendt 1997; Mangel and Stamps 2001) that may lead to negative correlations between growth and reproduction. Faster growth rates may increase survival during early transition phases; however, they may also incur costs that compromise later life stage performance (Gimenez et al. 2004, Gagliano et al. 2007). For example, the increased foraging effort required for high growth rates may limit the time available to defend spatial resources, such as oviposition sites. Regardless of whether the correlation between growth and reproduction is caused by direct selection for growth (e.g. good genes), co-variance with selected traits, or trade-offs, investigating the effects of growth and body size together can provide insight into how growth rates interact with male morphological traits to explain variability in male reproductive success.

In this study, I examine how early growth trajectories (derived from growth increments on fish scales) and current male traits explain variation in reproductive output between individuals in a temperate marine fish, Forsterygion lapillum. I hypothesize that growth rates will be positively correlated with reproductive output if growth rates are either (i) directly selected for by females or (ii) co-vary with other environmental resources, such as habitat quality. Alternatively, I expect that growth rates will be negatively correlated with reproductive output if somatic growth trades-off with reproductive output. In order to examine these 
hypotheses I measured the reproductive success of breeding males in relation to their size and growth rates over the breeding season at two different spawning locations.

\subsection{Methods}

\subsubsection{Field Collections}

In order to explore how variation in male reproductive success varies with male morphological traits and previous growth, I collected reproductive males and their clutches at regular intervals during the breeding season. In this way, I estimated the reproductive success for each male at a single time point during the breeding season, by quantifying clutch size (number of eggs in the nest) and egg size in each nest at the time of collection. I chose to sample male reproductive success once rather than longitudinally through time because of the logistic constraints of accurately locating males repeatedly throughout the breeding season. F. lapillum is an asynchronous, continuous spawner that may lay eggs daily throughout the season (Warren 1990); therefore, I assumed that reproductive success of individuals across the population could be estimated reasonably accurately from a single time point because there would be a consistent turnover of eggs. I collected 109 males and nests in total, from two sites located within the Wellington Harbour, New Zealand -- Moa Point $(\mathrm{n}=55)$ and Kau Bay ( $\mathrm{n}=54)$. Kau Bay (41 ${ }^{\circ} 17^{\prime}$ $18^{\prime}$ 'S 174\%49'54'E) is a north facing bay protected from strong southerly swells, while Moa Point (41 20 '34"S $\left.174^{\circ} 48^{\prime} 42^{\prime \prime} \mathrm{E}\right)$ is a more exposed south facing site exposed to southerly winds and swells. Sites were selected because they had large amounts of habitat suitable for triplefin reproduction (loose cobbles) and their orientation allowed paired sampling under most weather conditions. I sampled each site at roughly monthly intervals during the breeding season (September-December 2011) on paired sampling dates which were never separated by more than four days (Kau Bay - 20 Sept, 27 Oct, 30 Nov, Moa Point- 22 Sept, 31 Oct, 1 Dec).

On each sampling date, I haphazardly (i.e. without and formal method of randomization) established three $5 \mathrm{~m} \times 5 \mathrm{~m}$ quadrats in shallow $(1.2 \pm 0.4 \mathrm{~m}$, mean depth $\pm \mathrm{SE})$ cobble habitats, where adult $F$. lapillum were common. Within each quadrat, dive teams on SCUBA searched for nests of F. lapillum by haphazardly overturning cobbles. I attempted to collect 6 nests from each 
quadrat; however, occasionally less than six nests were found (average nests collected per quadrat $=5.7$ nests \pm 0.2 , mean $\pm \mathrm{SE}$ ). When a nest was located, the associated male was identified by its breeding colouration and exhibition of nest guarding behaviour. The depth and position of the nest within the quadrat were recorded and the nesting cobble was placed in a container with fresh seawater (one nest per bucket). Immediately after removal of the nest, divers collected the parental male that was guarding the clutch using a dip net, and placed it in the same container. Males of other triplfin species guard one nesting site at a time, until all eggs have hatched (Thompson 1986) and during the surveys, no males were found defending more than one nest suggesting that during sampling I was likely able to obtain all of the eggs from that paternal male. I did not attempt to collect males that were not defending a clutch (i.e. nonparental males) because my primary aim was to investigate variation in clutch size among males and not the differences in traits between males with and without nests. Containers with males and their clutches were transported a short distance (15 minutes via vehicle) to the Victoria University Coastal Ecology Laboratory (VUCEL). At the laboratory, an aerator (one for each container) was placed under the eggs to maintain proper oxygen levels. Parental males were removed from the containers and individually euthanized in an ice-sea water slurry. The total length (from snout tip to edge of the caudal fin, TL) and wet weight of each fish was measured, and males were then frozen until needed for further analysis. I calculated body condition using Fulton's K (Ricker 1975):

$$
\mathrm{K}==\frac{\text { wet weight }}{T L^{3}}
$$

\subsubsection{Measurement of egg number and egg size}

To measure reproductive success in individual males, I quantified both clutch size (egg number) and average egg size for each nest. Egg size can be important in determining larval survival and may be indicative of maternal condition (Brooks et al. 1997); therefore, I hypothesized that egg size may vary among paternal males due to variation in the quality of the females they attract. I counted egg number by briefly removing each clutch rock from its container, positioning it with the side containing the clutch facing upwards, and photographing the entire rock so that individual eggs were easily identifiable. I counted all the eggs in each

clutch manually using the photo analysis software, Image Pro Plus ${ }^{\circledR}$ (v5.0). I estimated average 
egg size for the entire clutch by haphazardly selecting 10-15 eggs, gently transferring them onto a glass slide using forceps, and photographing eggs at $8 \mathrm{X}$ magnification under a dissecting scope. Eggs were selected non-randomly across the entire clutch in order to get a representative sample of egg size from each nest. Using Image Pro Plus ${ }^{\circledR}$, I haphazardly selected two diameters at perpendicular angles for each egg, measured them, and then averaged both to obtain the overall egg size (i.e., variation between diameter measurements was ignored in subsequent analysis). Due to the difficulty in removing eggs from some nests undamaged, I was unable to estimate egg size from all clutches; however, I successfully sampled eggs from over $80 \%$ of the nests collected.

Due to the fact that F. lapillum males may mate with several females (Warren 1990), it is likely that my estimates of clutch size and egg size contained eggs of various ages from multiple females. Different clutches in male nests may be identified by using the developmental stage of the eggs (Thompson 1986); however, it is not possible to accurately differentiate between clutches that have been laid within close proximity of each other (e.g. hours or days). As $F$. lapillum is a continuous daily spawner it is likely that new clutches may be added daily (Warren 1990). Therefore, I limited measurements of reproductive success to (i) total clutch size and (ii) overall egg size and did not attempt to quantify the number of different clutches per nest or select eggs from similar clutches.

\subsubsection{Estimating growth history}

In order to examine the effects of previous growth histories on reproductive success, I analyzed intercirculus spacing in fish scales to obtain estimates of previous growth. Fish scales are made up of a combination of collagen fibers and a calcium deficient hydroxyapatite mineralized layer (Ikoma et al. 2003) that has overlapping layers imbedded in the dermis (Jawad 2005). Scales are commonly used in fisheries research to reconstruct growth histories and in age determination (Campana 2001; Cheung et al. 2007; Zymonas and McMahon 2009; Herbst and Marsden 2011) and the strong relationship between scale radius and total length can be used to back-calculate size (Takahashi 2008). Circuli (scale rings) are formed on the anterior edge of the growing scale and the distance between circuli (intercirculus spacing) is usually proportional to growth rates (Fisher and Pearcy 1990; Friedland 2000; Wells et al. 2003; Fisher and Pearcy 
2005) and therefore can be used to estimate growth per increment rates. In triplefins, they can be used to predict differences in growth between individuals (individuals $<40 \mathrm{~mm}$, Chapter 3), with greater intercirculus spacing indicating greater growth rates.

The majority of triplefin scales are ctenoid and are found covering most of the body (Jawad 2005). During the lifetime of the fish, scales may be lost and replaced by regenerated scales. These regenerated scales are often deformed and do not contain any growth information prior to scale loss; therefore, I only collected non-regenerated scales for growth analysis. I collected 2 to 4 non-regenerated scales from the region of the body directly posterior to the pectoral fin below the lateral line (see Fig. 3.1A). Scales from this region can be easier to read in some fish (Takahashi 2008) and in triplefins, scales from this area share a similar squamation pattern (size and growth type of scales, Jawad 2005) allowing for consistency in increment analysis. In addition, I chose scales that were behind the pectoral fins because I hypothesized that these scales would be more protected from scale loss, which can be increased during the reproductive season in nest guarding fish (Fouda 2009) due to physical damage associated with the parental care (Sabat 1994). After removal, I lightly cleaned scales in deionized water, placed scales inside an Eppendorf tube, and used a sonicator to remove any tissue or pigment remaining on the scales. I then mounted scales between two glass slides in a drop of immersion oil and photographed them at 50X magnification using a compound microscope. For 18 males of the 109 collected, I was unable to obtain any non-regenerated scales from this region and therefore I could not obtain standardized growth estimates for these fish. One male from Kau Bay was removed from the study because the TL of the fish $(78.05 \mathrm{~mm})$ was more than $2 \mathrm{SD}$ above the mean for Kau Bay and the body of the fish appeared to be deformed.

Fish scales form around the time fish settle; however, when they are initially fully formed scales may already have some circuli and the relationship between the distance of these initially formed circuli and growth are unknown. I conducted a preliminary study to determine (i) the total length where scale formation was occurring, (ii) which circuli on the scale to start measurements from, and (iii) what total length corresponded to the selected circuli (i.e. where was somatic growth measured from, details in Appendix B). Based on results from the preliminary study, I chose to measure intercirculus spacing starting at the $6^{\text {th }}$ intact circuli (to avoid potentially dubious measurements of circuli formed during initial scale formation prior to 
settlement). I estimated the average intercirculus spacing between the $6^{\text {th }}$ circuli and the $16^{\text {th }}$ circuli (10 individual measurements of intercirculus space) and then over the next 10 circuli (circuli 17-27) to investigate how the importance of growth to reproduction varied between these two different regions (Fig 4.1). For each fish, I measured all collected scales and then averaged them to get an estimate of growth for each fish.

I estimated the approximate size ranges covered by these two sets of circuli using a backcalculation technique (biological intercept method, Campana 1990). I used $27 \mathrm{~mm}$ as an initial size at scale formation because the probability of scale formation was greater than $80 \%$ based on the preliminary study at this length (Appendix B). I used an initial scale size of size of $323 \mu \mathrm{m}$ (estimated from my preliminary study, Appendix B). Results indicated that circuli 6-16 covered growth from settlement up until approximately $33 \mathrm{~mm} \pm 0.1$ (mean \pm SE) for Kau Bay and to 34 $\mathrm{mm} \pm 0.1$ for Moa Point (sites are different because of increased intercirculus spacing at Moa Point). Circuli 17-27 covered growth from $33 \mathrm{~mm} \pm 0.1$ to $39 \mathrm{~mm} \pm 0.1$ for Kau Bay and from $34 \mathrm{~mm} \pm 0.1$ to $41 \mathrm{~mm} \pm 0.2$ for Moa Point. 


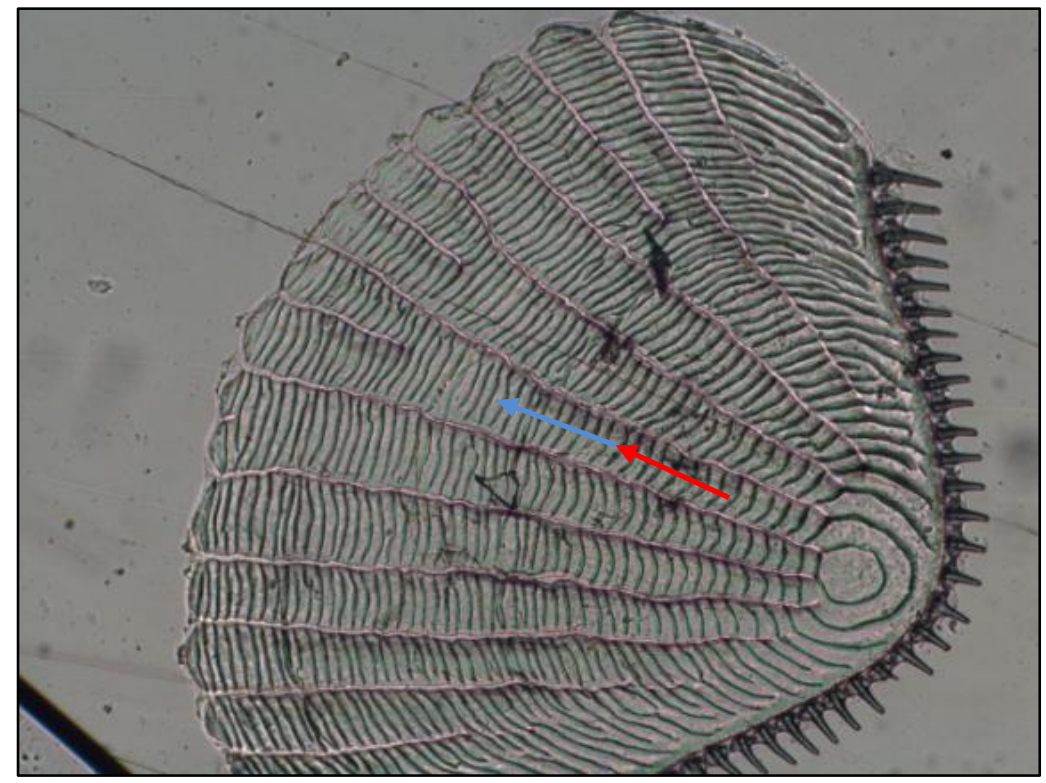

Figure 4.1 A non-regenerated scale from an adult common triplefin, Forsterygion lapillum (anterior side on the left and posterior on the right). In order to determine examine previous growth, intercirculus spacing was measured from the 6th circuli to the 16th (red line) and from the 17 th circuli to the 27 th (blue line). 


\subsubsection{Statistical analysis}

\section{Differences in male characteristics between spawning locations}

I examined how the traits of total length, wet weight, body condition, and intercirculus spacing differed between the two spawning locations using separate one-way ANOVAs with spawning location as an independent variable.

\section{The effect of male traits on reproductive output}

To explore the effects of male traits (including growth rate) on reproductive output as well as how these effects varied between different spawning locations over the breeding season, I used linear mixed effects models with response variables of clutch size and egg size. Preliminary analysis indicated that male traits of total length (TL) and growth per increment differed significantly between spawning locations, making the direct comparison of male traits and reproductive success problematic because differences in growth rate and TL across all samples would be biased by their spawning location. Therefore, instead of fitting the actual values for intercirculus spacing and TL as predictors in the model, I fit the residuals of those traits for each male at their respective spawning location separately (residualisation). I obtained residualized measures of TL and intercirculus spacing from separate one-way ANOVAs comparing traits between spawning locations (see Differences in male characteristics between spawning locations). In this way, the mean value for intercirculus spacing and TL for both spawning locations was equal to zero; therefore, males with larger relative growth compared to individuals at the same location had positive values and individuals with lower relative growth had negative values. This allowed for an unbiased analysis for the effects of male traits on reproductive output both between spawning locations and for all fish.

In order to test different hypotheses about the effects of male traits on reproductive success, I developed a set of 187 candidate models for clutch size (number of eggs) and egg size with different combinations of fixed effects including TL, body condition, intercirculus spacing (separate candidate sets for circuli 6-16 and circuli 17-27), time of spawning within the breeding season (September, October, November) and spawning location (Kau Bay and Moa Point). For all candidate models, I fit transect identity as a random factor due to the fact that males from the same transect may have courted or received eggs from the same females and therefore, would have violated the assumption of independent replicates. Mixed effects models can account for 
correlations between replicates by fitting the replicated unit (transect) as a random effect (Bolker et al. 2009). I compared all candidate models using information theoretic criterion and ranked each model based on AIC values (Akaike 1974) with a correction for small sample sizes (AICc), a commonly used method in ecology for model selection (Johnson and Omland 2004). All input variables were standardized in each model (scaled to have a mean of zero and a standard deviation of 1) in order to improve the interpretability of regression coefficients (Gelman 2007). Several models had $\triangle \mathrm{AICc}<2$, suggesting that there was high support for multiple models (Burnham and Anderson 2002). Therefore, I used a model averaging approach (see Appendix A) to make inferences across a selected group of top models by obtaining averaged parameter estimates for the fixed factors. To select a group of top models for model averaging, I used a cut-off criterion of $\triangle$ AICc $<2$ (Burnham and Anderson 2002; Grueber et al. 2011). Once model averaged parameters were obtained, I used Wald Z-scores to make inferences about each parameter estimate. I repeated this procedure, once for circuli 6-16, and once for circuli 17-27.

\section{Separating by sampling date}

To further investigate the effects of average intercirculus spacing on reproduction I conducted a second analysis where I separated each sampling date and performed model selection. For each sampling date the model included total length, body condition, intercirculus

spacing, and spawning location with all two-way interactions. I only chose to use circuli 6-16 in this analysis because results indicated that circuli 17-27 were less important for reproductive success.

\subsubsection{Statistical software}

All statistical analysis was performed in R ( $\mathrm{R}$ Core Team 2013). For linear models comparing male traits between sites, I used the R function "lm". Linear mixed effects models were done using the package "Ime4" with the function lme (Bates et al. 2013). Candidate model selection and model averaging was completed with the package "MuMIn" and the functions dredge, modavg, and predict.modavg (Barton 2013). 


\subsection{Results}

\subsubsection{Differences in male characteristics between spawning locations}

Several male traits differed between spawning locations with parental males from Moa Point having a larger TL, wet weight, and intercirculus spacing than males from Kau Bay on average (Table 4.1).

\subsubsection{Clutch size models}

AICc values suggested that there was support for several different models therefore, I used model averaging to make inferences across multiple models by obtaining parameter estimates based on several models together (Appendix A). For circuli 6-16, the averaged model contained 6 separate models and included fixed effects of intercirculus spacing, time of spawning, spawning location, and body condition, an interaction between time of spawning and intercirculus spacing, and an interaction term between time of spawning and body condition (Table 4.2). For circuli 17-27, the averaged model contained 11 models, but had the same fixed effects as the model for circuli 6-16 (Table 4.2).

The average number of eggs \pm SE per nest, pooled over the entire sample period was $1200 \pm 651$; however, reproductive output showed a significant seasonal decline with nests at the end of the season containing 37\% fewer eggs when compared with the first sampling date (Fig. 4.2, Table 4.2). Average intercirculus spacing was the only male trait that was significantly correlated with reproductive output (Table 4.2); however, in circuli 17-27 this relationship was diminished. The negative relationship appeared to be driven strongly by fish collected during the second sampling period (Table 4.3), but even during this period the amount of variation in egg number explained by average intercirculus spacing was low (conditional $\mathrm{R}^{2}=0.156$ ). For the second sampling period, I converted the standardized regression estimate (the effect of one standard deviation of the predictor on the response variable) into an associated increase in average intercirculus spacing. I found that egg number decreased by 236 (CI 40.73, 432.19) for every increase of $1.45 \mu \mathrm{m}$ in intercirculus spacing for Kau Bay, and for every $1.93 \mu \mathrm{m}$ increase in intercirculus spacing for Moa Point (Fig. 4.3). 
Table 4.1 Means \pm SE in male characteristics between Kau Bay and Moa Point for all males pooled across sampling dates. F statistics including degrees of freedom (df) and $p$ values are based on one way ANOVAs between each variable with an independent factor of spawning location

\begin{tabular}{lcccc}
\hline \multicolumn{1}{c}{ Variable } & Kau Bay & Moa Point & F statistic (df) & p \\
\hline \hline Total length $(\mathrm{mm})$ & $58.1 \pm 0.52$ & $62.7 \pm 0.56$ & $37.3(1,89)$ & $<0.001$ \\
\hline Wet weight $($ grams) & $2.02 \pm 0.06$ & $2.66 \pm 0.07$ & $43.73(1,89)$ & $<0.001$ \\
\hline Fulton's K & 0.000010 & 0.000011 & $2.83(1,89)$ & 0.0839 \\
\hline Circuli 6-16 (mean $\mu \mathrm{m})$ & $17.63 \pm 0.25$ & $19.42 \pm 0.26$ & $24.99(1,89)$ & $<0.001$ \\
Circuli 17-27 (mean $\mu \mathrm{m})$ & $16.96 \pm 0.21$ & $18.60 \pm 0.22$ & $28.81(1,89)$ & $<0.001$ \\
\hline
\end{tabular}




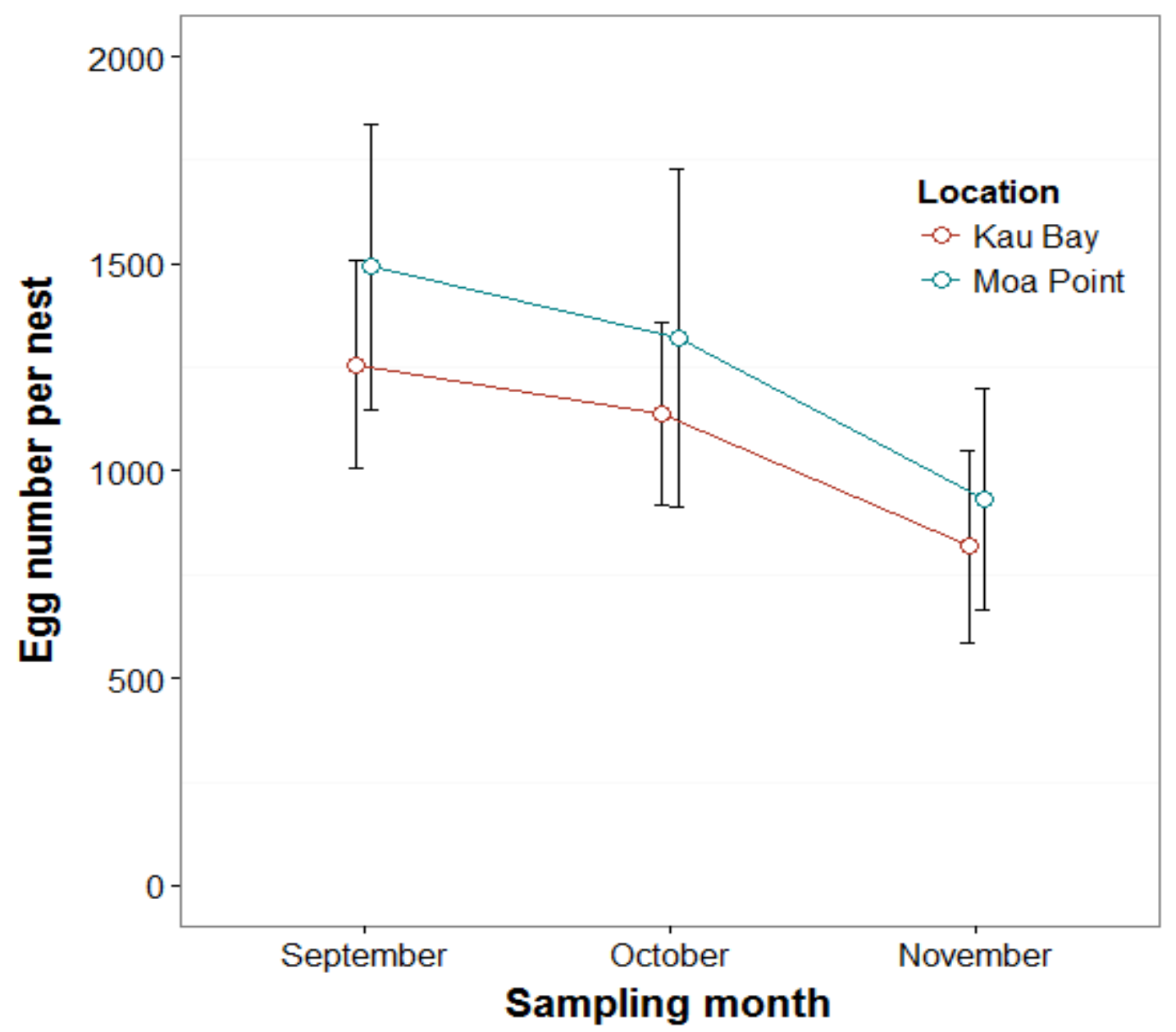

Figure 4.2 The effect of time of spawning on clutch size over the breeding season for Forsterygion lapillum males collected from two different spawning locations (represented by two different coloured lines). Errors bars are 95\% confidence intervals. The third sampling period (November) is significantly different from the two samples taken earlier in the breeding season (locations pooled). 
Table 4.2 Standardized (input variables scaled to a mean of 0) model-averaged regression estimates for models with a response variable of clutch size including averaged intercirculus spacing between scale circuli 6-16 or circuli 17-27 fit as a fixed factor. Unconditional standard errors (SE) include model selection uncertainly in their estimates. Upper and lower confidence intervals (CI) are given on the regression estimates. Relative importance (RI) gives the sum of the AICc weight for each model that parameter was included in (a RI of 1 indicates that parameter was present in all of the averaged models). For the interaction between sampling period and intercirculus spacing and between sampling period and Fulton's K individual regression estimates are given for each level of sampling period (e.g., Sample 2, Sample 3), but in all cases the models included interactions between all levels of sampling period. P-values are based on Wald test $Z$ scores with significant values given in bold. Averaged model for circuli 6-16 included estimates from 6 models and averaged model for circuli 17-27 included estimates from 9 models.

\begin{tabular}{|c|c|c|c|c|c|c|c|}
\hline Circuli & Fixed effects & $\begin{array}{c}\text { Regression } \\
\text { estimate }\end{array}$ & RI & $\begin{array}{c}\text { Unconditional } \\
\text { SE }\end{array}$ & Lower CI & Upper CI & p-values \\
\hline \multirow{10}{*}{6 to 16} & Sampling 1 (Sept) -Intercept & 1357.91 & 1.00 & 89.37 & 1182.75 & 1533.07 & $<0.001$ \\
\hline & Sampling 2 (Oct) & -179.45 & 1.00 & 131.92 & -438.07 & 79.17 & 0.174 \\
\hline & Sampling 3 (Nov) & -425.17 & 1.00 & 159.60 & -737.99 & -122.361 & 0.007 \\
\hline & Fulton's K & 120.36 & 0.47 & 951.25 & -739.06 & 2989.77 & 0.237 \\
\hline & Intercirculus spacing & -132.97 & 1.00 & 64.42 & -259.23 & -6.72 & 0.039 \\
\hline & Spawning location & -84.44 & 0.36 & 59.35 & -200.75 & 31.88 & 0.155 \\
\hline & Sample $2 \times$ Intercirculus spacing & -67.15 & 0.11 & 127.09 & -316.24 & 181.94 & 0.597 \\
\hline & Sample $3 \times$ Intercirculus spacing & 261.92 & 0.11 & 179.34 & -89.59 & 613.42 & 0.144 \\
\hline & Sample $2 \times$ Fulton's K & -226.92 & 0.10 & 140.24 & -501.803 & 47.94 & 0.106 \\
\hline & Sample $3 \times$ Fulton's $\mathrm{K}$ & 22.99 & 0.10 & 240.02 & -447.44 & 493.43 & 0.924 \\
\hline \multirow{10}{*}{17 to 27} & Sampling 1 (Sept) -Intercept & 1357.61 & 1.00 & 87.61 & 1185.91 & 1529.32 & $<0.001$ \\
\hline & Sampling 2 (Oct) & -144.14 & 1.00 & 130.62 & -400.14 & 111.86 & 0.270 \\
\hline & Sampling 3 (Nov) & -473.74 & 1.00 & 155.21 & -777.94 & -169.54 & 0.002 \\
\hline & Fulton's K & 112.62 & 0.46 & 92.31 & -68.32 & 293.55 & 0.222 \\
\hline & Intercirculus spacing & -134.49 & 1.00 & 71.68 & -274.98 & 5.99 & 0.060 \\
\hline & Spawning location & -85.64 & 0.39 & 58.00 & -199.33 & 28.04 & 0.140 \\
\hline & Sample $2 \times$ Intercirculus spacing & -81.80 & 0.33 & 124.20 & -325.22 & 161.62 & 0.510 \\
\hline & Sample $3 \times$ Intercirculus spacing & 269.75 & 0.33 & 173.75 & -70.77 & 610.30 & 0.121 \\
\hline & Sample $2 \times$ Fulton's K & -216.05 & 0.07 & 138.11 & -486.73 & 54.63 & 0.117 \\
\hline & Sample $3 \times$ Fulton's K & -21.75 & 0.07 & 236.15 & -441.08 & 484.60 & 0.926 \\
\hline
\end{tabular}


Table 4.3 Standardized (input variables scaled to a mean of 0) model-averaged regression estimates (Sample 1 and Sample 3 ) or mixed model regression estimates (Sample 2) from models for each sampling period. Intercirculus spacing is for circuli 6-16. Unconditional standard errors (SE) include model selection uncertainly in their estimates. Upper and lower confidence intervals (CI) are given on the regression estimates. Relative importance (RI) gives the sum of the AICc weight for each model that parameter was included in (a RI of 1 indicates that parameter was present in all of the averaged models). P-values for sample 1 and sample 3 are based on Wald test $\mathrm{Z}$ scores, while sample 2 is based $t$ values. Significant values are given in bold.

\begin{tabular}{cccccccccc}
\hline \multirow{2}{*}{ Sampling time } & Fixed effects & $\begin{array}{c}\text { Number of } \\
\text { models }\end{array}$ & $\begin{array}{c}\text { Regression } \\
\text { estimate }\end{array}$ & RI & $\begin{array}{c}\text { Unconditional } \\
\text { SE }\end{array}$ & Lower CI & Upper CI & p-values \\
\hline \hline Sample 1 & Fulton's K & \multirow{2}{*}{4} & 257.65 & 0.61 & 1481.9 & -57.16 & 4875.10 & 0.056 \\
& & Intercirculus spacing & & -130.22 & 0.37 & 104.4 & -334.78 & 74.35 & 0.212 \\
& Sample 2 & Intercirculus spacing & 1 & -236.46 & - & - & -432.19 & -40.73 & $\mathbf{0 . 0 2 6}$ \\
\hline & Sample 3 & Intercirculus spacing & 2 & 129.07 & 0.22 & 98.47 & -80.85 & 355.75 & 0.217 \\
\hline
\end{tabular}




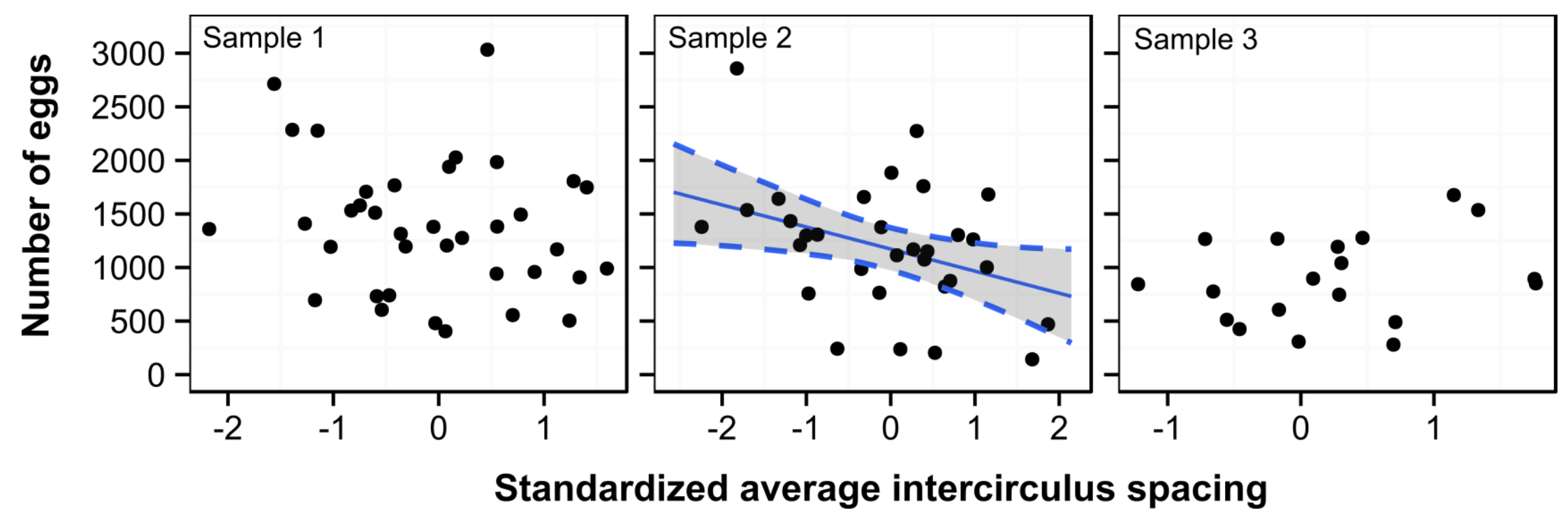

Figure 4.3 Relationship between clutch size (the number of eggs in a nest at sampling) and standardized intercirculus spacing (i.e., individual growth relative to other fish at that site) in F. lapillum for the three sampling periods (September, October, and November). To ease visualization, other model covariates were fixed to their means. Standardized growth values (based on average intercirculus spacing over circuli 6-16) were calculated separately for each site (Moa Point and Kau Bay) because growth rates differed between sites. Positive standard growth values indicate that an individual grew faster than average at that site, while a negative value indicates that males grew slower than average at that site. Each point represents a single nest and the corresponding male $(\mathrm{n}=93)$. The November sampling period was the only time when standardized average intercirculus growth correlated significantly with the number of eggs in a clutch. Grey area around the mean represents $95 \%$ confidence intervals on the regression line fit. 


\subsubsection{Egg size model selection}

As with clutch sizes, there was not strong evidence indicating that one single model described the data well. I used the same procedure to select a subset of top models (AICc values $<\Delta 2$ ) and average their parameter estimates. The averaged model included the fixed effects of time of spawning, body condition, spawning location, and a significant interaction between time of spawning and body condition. Body condition did have a significant effect on egg size, although this varied significantly with time of spawning (Table 4.4). 
Table 4.4 Model-averaged standardized regression coefficients, unconditional standard errors, confidence intervals, and $\mathrm{p}$ values (based on Wald z scores) for fixed effects in the egg size model. Sample one is the intercept and all other estimates are the effects on average egg size based on an increase of one standard deviation in the fixed variable. Unconditional standard errors take into account model uncertainty across several models. Percent change in mean egg size is taken from the average for sampling one (the intercept) for time of spawning and location. Percent change for Fulton's K is taken from the average of the appropriate sampling period.

\begin{tabular}{lcccccc}
\hline \multicolumn{1}{c}{ Fixed effects } & $\begin{array}{c}\text { Standardized } \\
\text { estimate }\end{array}$ & $\begin{array}{c}\text { Unconditional } \\
\text { SE }\end{array}$ & $\begin{array}{c}\text { Lower } \\
\text { CI }\end{array}$ & $\begin{array}{c}\text { Upper } \\
\text { CI }\end{array}$ & $\begin{array}{c}\text { p } \\
\text { values }\end{array}$ & $\begin{array}{c}\text { Change in } \\
\text { individual egg } \\
\text { diameter (\%) }\end{array}$ \\
\hline \hline Sample 1 (Sept) & 1.117 & 0.008 & 1.101 & 1.133 & $<\mathbf{0 . 0 0 1}$ & 0.00 \\
Sample 2 (Oct) & -0.044 & 0.012 & -0.068 & -0.021 & $<\mathbf{0 . 0 0 1}$ & -3.90 \\
Sample 3 (Nov) & -0.058 & 0.013 & -0.083 & -0.032 & $<\mathbf{0 . 0 0 1}$ & -5.10 \\
$\begin{array}{l}\text { Fulton's K } \\
\text { (Sample 1) }\end{array}$ & -0.039 & 0.017 & -0.073 & -0.006 & $\mathbf{0 . 0 2 2}$ & -3.40 \\
$\begin{array}{l}\text { Fulton's K } \\
\text { (Sample 2) }\end{array}$ & 0.022 & 0.019 & -0.016 & 0.059 & 0.261 & 2.00 \\
$\begin{array}{l}\text { Fulton's K } \\
\text { (Sample 3) }\end{array}$ & 0.100 & 0.029 & 0.044 & 0.156 & $<\mathbf{0 . 0 0 1}$ & 9.40 \\
$\begin{array}{l}\text { Spawning location } \\
\text { Saln }\end{array}$ & 0.011 & 0.010 & -0.009 & 0.031 & 0.279 & 0.09
\end{tabular}




\subsection{Discussion}

Examining the male traits that influence variation in male reproductive output can help predict patterns of reproductive success among individuals. In this study, I aimed to identify factors, including previous growth histories, which were driving male variation in reproductive success in the common triplefin. Clutch size (number of eggs per nest) was highly variable among individuals over the study period; however, I detected a significant, albeit subtle relationship between clutch size and early growth (measured by scale increment width). Although growth explained relatively small amounts of total variation, it was the only male trait I measured that significantly correlated with clutch size. Males that had a higher standardized growth rate (i.e., grew faster relative to other fish at that location) had fewer eggs on average per clutch. Negative correlations between clutch size and growth rates during this period may have been caused by several possible mechanisms. For example, F. lapillum is extremely site attached (Clements 2003, Smith 2010), usually occupying a small habitat patch throughout ontogeny, and certain habitat types where juvenile growth is high (e.g. such as particular macroalgal stands, Smith 2010), may contain fewer high-quality nesting sites than habitats where juvenile growth is slower. If females choose males based on the quality of their oviposition sites (Petersen 1988), male reproductive success would decline with decreasing reproductive habitat quality. I was unable to age individuals, but the observed effects of growth rate could have been be caused by differences in settlement date. Juveniles with faster growth rates may have settled later in the recruitment season, when increased temperatures may allow for faster growth (Sponaugle et al. 2006). However, their reproductive output may be reduced because of increased competition with established residents, which may force them to occupy marginal reproductive habitats. Alternatively, the presence of a negative relationship between early growth and later reproductive success could indicate that trade-offs between somatic growth and reproduction may be important in determining variation in reproductive success. For instance, survival during the early transition phases can be strongly influenced by size via size-selective mortality for many reef fish (Sogard 1997). In F. lapillum, smaller settlers may experience increased mortality rates post-settlement (Smith 2010) and cannibalism by resident adults may be restricted to smaller settlers (Chapter 2). Faster somatic growth rates may therefore be selected for during early growth, but may also come with an associated cost that reduces reproductive output (e.g. increase foraging time). Female mate preferences may vary over the breeding 
season (Oliveira et al. 1999), which may explain the temporal variation in the response of reproductive success to growth rate. In addition, my survey design does not account for the possibility that individuals with faster growth rates may have bred for a longer period or more frequently via effects on age and size at sexual maturity (Stearns 2000). Although variation in growth rate explained small amounts of variation in overall reproductive success, these results show that growth rates can influence reproductive success, regardless of other male phenotypic traits.

Size is often one of the most important predictors of reproductive success in fish species (Thompson 1986); however, I found no relationship between reproductive output and male size (TL and wet weight) or body condition, even when statistically accounting for the effects of previous growth histories. Size is often correlated with reproductive success in males because size may influence competition for access to females or nesting resources (Hastings and Petersen 2010). In nest breeders, such as F. lapillum, the effects of body size on the ability of males to monopolize resources can depend upon the availability and distribution of nests (Reichard et al. 2009). For example, where reproductive habitat is scarce and is spatially clumped, larger males may be able to use their competitive advantage to monopolize mating resources and obtain the majority of mating opportunities (Kelly 2008). For the purposes of this study, I specifically chose two locations (Moa Point and Kau Bay) that had a high availability of nesting habitats (cobbles), and in these areas competition for oviposition sites may have been quite low, reducing any benefits of increased size. Furthermore, I only investigated males that had obtained eggs (i.e. those that were already defending an oviposition site) and body size may be more important in determining access to oviposition sites, and have less of an influence on variation in clutch size among males who have already obtained an oviposition site. This feature of the study design may also explain why the main effects in my statistical analysis described such low amounts of variation in clutch size.

Clutch size appeared to steadily decline over the breeding season, and was significantly smaller at the end of the breeding season for both sites. Steady declines in clutch size have been observed for other demersal spawners (Oliveira et al. 1999), and may be caused by seasonal variation in maternal reproductive effort. Females may invest more heavily in spawning at the beginning of the season, as spawning date can significantly affect offspring survival, with larval 
fish spawned early in the breeding season usually having a higher probability of survival (Cargnelli and Gross 1996). In addition to reductions in clutch size, egg size at Kau Bay also declined over time. Seasonal reductions in egg size are common to several fish species, and are thought to be caused by either (i) active changes in maternal provisioning by females over time (Semmens and Swearer 2012), (ii) exhaustion of energy reserves at the end of the season, or (iii) indirect effects on reproduction caused by environmental changes (King et al. 2003). Females may be adaptively responding over the breeding season by reducing their egg size in coordination with improving environmental conditions (warmer temperatures) later on in the spring (Castro et al. 2009). Interestingly, Moa Point did not appear to show the same seasonal decrease in egg size. Differences between the two locations potentially indicate that the quality and/or dispersive capabilities (higher quality larval fish may be able to disperse for longer distances) of larvae from the different sites may change over the breeding season. Previous studies on $F$. lapillum have shown that larval environments heavily influence survival probabilities at settlement, with individuals who develop within the harbour having a survival advantage over individuals that developed on the south coast (Shima and Swearer 2010). I cannot attribute variation in egg size to differences in locations as my study was unreplicated within regional locations; however, if seasonal effects on egg size are variable between locations, it may indicate that the relative contribution of different locations to overall recruitment may also vary seasonally via differential survival of high quality larvae (originating from larger eggs). Temporal variation in both egg number and egg size may have implications for variation in male reproductive success over the season via changes in offspring number and mortality, which may ultimately affect recruitment dynamics.

Overall, variation in reproductive output appears to be large, and may be the result of several complex spatial and temporal processes. However, the observed effect of previous growth on variation in reproductive output among individuals potentially suggests that reproduction may be influenced by growth occurring at a critical time (i.e., settlement). The relative importance growth rates on reproduction appear to vary with time, and may potentially interact with seasonal declines in clutch and egg size to modify the reproductive success of individual males. 


\section{Chapter 5 - Reproductive success of parasitised males in a marine reef fish}




\subsection{Abstract}

Parasitism is hypothesized to reduce reproductive success in heavily parasitized males because females may preferentially mate with unparsitised males (parasite-mediated sexual selection) or parasites may compromise male competitiveness. In marine systems, this hypothesis is largely unexplored. This paper provides the first confirmed record of a copepod ectoparasite (Caligus buechlerae Hewitt 1964) on the common triplefin (Forsterygion lapillum) and evaluates the hypothesis that males parasitized with C. buechlerae experience lower reproductive success than unparasitised males (as determined by the presence and area of eggs within male nests). I found that $38 \%$ of males I surveyed were infected with at least one C. buechlerae, with a median of 2 individuals per infected male. $32 \%$ of males were defending eggs, with $62.5 \%$ of those males infected with at least one parasite. Males of greater total length were both more likely to be infected and more likely to be defending eggs. However, when statistically accounting for the effects of total length, parasite infection had no effect on the probability of defending eggs, or the average surface area of eggs when present. Positive covariation in fish length, the presence of eggs, and parasite infection observed here potentially suggest that the importance of parasitic infection on reproductive success may depend upon the strength of selection for larger male body size. My study is one of the few studies to investigate the effects of ectoparasites on reproductive success in reef fish, and also provides a quantitative measure of infection for a widespread species within New Zealand. 


\subsection{Introduction}

After the initial proposal by Hamilton and Zuk (1982) that suggested parasitism might strongly influence patterns of sexual selection, the role of parasites in mediating reproductive success has been extensively studied (Howard and Mincheulla 1990; Clayton 1991; Hamilton and Poulin 1997; Møller et al. 1999). Parasites may influence male reproductive success via two mechanisms. First, if parasite prevalence and virulence within a host population are high, females may evolve mechanisms to detect and avoid mating with parasitized males (Endler and Lyles 1989; Clayton 1991; Clayton et al. 1992; Poulin and Vickery 1993) in order to (i) limit their exposure to contagions during breeding (parasite avoidance hypothesis, Able 1996, Hillgarth 1996), (ii) confer parasite resistance to their offspring (good genes hypothesis, Hamilton and Zuk 1982; Barber et al. 2001), or (iii) increase parental care (the efficient parent hypothesis, Hakkarainen et al. 1998; Møller et al. 1999). Second, parasite infection may reduce male reproductive success by negatively affecting male competitiveness for mating resources and opportunities (Howard and Mincheulla 1990). The disproportionate distribution of reproductive success between parasitised and unparasitised males may have implications for the genetic diversity of populations (Hamilton 1982) and, if selection against parasitism is strong, it may influence the evolution of host populations over time. The majority of research measuring the effect of parasitism on reproductive success has originated from terrestrial (Zuk et al. 1990; Forbes 1991; Ehman and Scott 2002; Webster et al. 2003) or freshwater habitats (Milinski and Bakker 1990; Houde and Torio 1992; Poulin 1994), with less emphasis on marine environments. Parasitism in marine ecology demands more attention, especially since the long-range dispersal patterns of many marine host species may cause infection to spread more rapidly compared to other systems (McCallum et al. 2004).

Marine reef fish may experience a high prevalence of ectoparasite infection (Grutter 1994, Rohde et al. 1995), with current research indicating that ectoparasites can influence the demography of reef fish via deleterious effects on host physiology (Adlard and Lester 1994, Finley and Forrester 2003, Binning et al. 2013). However, few studies have explored the potential for ectoparasite infection to mediate male reproductive success in reef fish and results from previous studies have been equivocal. For example, two studies found no observable effect of ectoparasite infection on male reproductive success (multiple ectoparasites on sand gobies - 
Barber 2002; gnatid isopods on longfin damselfish - Cheney and Côté 2003), and one study (Rosenqvist and Johansson 1995), found that pipefish males avoided mating with females harbouring parasitic trematodes (which caused large black spots on the female). Barber (2002) speculated that perhaps parasite effects on host reproductive success may be more likely if parasites directly alter the male phenotype (e.g. spots - Rosenqvist and Johansson 1995), presumably because changes in phenotype may facilitate visual detection of parasitized males by females. When females are unable to determine male quality via visual cues, mate choice decisions can vary substantially (Heuschele et al. 2009), which may potentially weaken or even reverse the influence of parasites on reproductive success (Heuschele and Candolin 2010). Furthermore, evidence from freshwater fish suggest that visual determination of parasite infection is important (Barber 2013), with females avoiding parasitized males when the male phenotype is clearly altered by infection (Milinski and Bakker 1990, Tobler et al. 2006), but not when phenotypic effects are unobserved (Sparkes et al. 2013).

In marine systems, copepod ectoparasites of the Caligidae family (sea lice) infect many fish species (Boxshall 1974, Johnson et al. 2004, Costello 2006), and their relatively large size ( 4-5 $\mathrm{mm}$ in length) may allow females to visually assess parasite load directly, which in turn, may favour the evolution of parasite-mediated sexual selection (Endler and Lyles 1989).

Furthermore, Caligid ectoparasites may have strong negative effects on the condition and growth of their hosts (Costello 2006; Costello 2009), which could subsequently influence male reproductive success via deleterious effects on competitive abilities or courtship behaviour (Howard and Micheulla 1990; Pélabon et al. 2005). In the present study, I investigated the effects of infection by a Caligid ectoparasite (Caligus buechlerae) on male reproductive success in the common triplefin (Forsteryion lapillum), a nest-guarding temperate reef fish. In nestguarding reef fish, larger males often experience increased reproductive output (Oliveira et al. 1999); however, the probability of ectoparasite infection may also increase with size for reef fish (Grutter 1994). Here, I used field surveys to evaluate the hypothesis that infected males have lower reproductive success (as determined by the presence of eggs and egg area) than uninfected males, while also accounting for the potential effects of body size on infection status. 


\subsection{Methods}

\subsubsection{Study species}

The common triplefin is a small reef fish (maximum total length $\sim 70 \mathrm{~mm}$ ) of the Family Tripterygiidae (triplefins) and is one of the most common benthic fish in shallow coastal areas around New Zealand (Willis 2001; Wellenreuther et al. 2007a; Wellenreuther et al. 2008). The species is an asynchronous continuous spawner, that may lay eggs daily (Warren 1990) throughout the breeding season (September-December in my study area). During the breeding season, males are typically site attached (Clements 2003), assume dark black nuptial colours (Wellenreuther and Clements 2007), display elaborate courtship behaviour (Handford 1979), and defend breeding territories within cobble habitats (Warren 1990). Unlike males, females move greater distances during the breeding season to find mating opportunities (Clements 2003) and typically lay eggs underneath cobbles within the territory of males (Feary and Clements 2006). Males remain close to the nest entrance during defense (Warren 1990) and may defend clutches of eggs deposited by multiple females, with larvae hatching after approximately 3 weeks (Francis 2012). At present, only two parasites are known to infect triplefins: the intestinal tape worm, Opegaster gobii, and a copepod ectoparasite, Caligus buechlerae (Jones 1988, Hine et al. 2000).

Caligus buechlerae Hewitt 1964 is a copepod ectoparasite found on the body surface of triplefins and other fish species in New Zealand (Hewitt 1964; Jones 1988, Fig. 5.1). To determine ectoparasite identity, I collected copepod ectoparasites from male $F$. lapillum at my study site three weeks before my field surveys. Ectoparasites were identified as Caligus buechlerae due to the presence of a bifurcated second maxillae, a characteristic unique to this Caligus species (Hewitt 1964). Any subsequent observations of copepod ectoparasites on triplefins at my study site were assumed to be $C$. buechlerae, given that it is the only known ectoparasite to infect triplefins in New Zealand (Hine et al. 2000). This paper provides the first confirmed record of $C$. buechlerae on the common triplefin and the first measurements of its prevalence in New Zealand, but species-specific information on the ecology, life span, contagiousness between hosts or pathogenic effects of C. buechlerae is currently limited.

Planktonic Caligus larvae ( 0.6 mm long, González and Carvajal 2003) attach to their hosts and remain sessile until they reach maturity (Costello 2006), which takes $\sim 12$ days post 
infection at $14^{\circ} \mathrm{C}$ (González and Carvajal 2003). As adults ( 4-5 mm long - González and Carvajal 2003, Bravo 2010), Caligus spp. freely move around the body surface of the host feeding on mucous, scales, blood or skin tissue (Johnson et al. 2004, Costello 2006). Caligid infections may cause wounds or skin lesions (Johnson et al. 2004, Costello 2006), which can increase host susceptibility to secondary infections or diseases (Mustafa et al. 2000b). Life spans of Caligid spp. are strongly temperature-dependent, but available evidence suggests that individuals may survive anywhere from 10 weeks (Bravo 2010) up to 7 months on their host (Mustafa et al. 2000a). Adult Caligus may be able to survive away from their host for up to 7 days (Bravo 2010); although, the ability of mature parasites to laterally transfer between hosts is unknown.

Caligus buechlerae is a medium-sized ectoparasite (males $\sim 3.7 \mathrm{~mm}$ long, females $\sim 5 \mathrm{~mm}$ long, Hewitt 1964) and triplefins regularly consume elusive prey items which are smaller or similar in size to C. buechlerae (e.g., ostracods, gammarid amphipods, Feary et al. 2009); therefore, I assume that the visual acuity of females should be sufficient to detect $C$. buechlerae infections. Furthermore, I suggest that the black breeding colouration of $F$. lapillum males increases the visual contrast with lighter coloured $C$. buechlerae, which may facilitate the direct detection of parasites on males by females (e.g., Borgia 1986), in addition to any indirect signals of parasite infection (e.g., colour change, Milinski and Bakker 1990). 

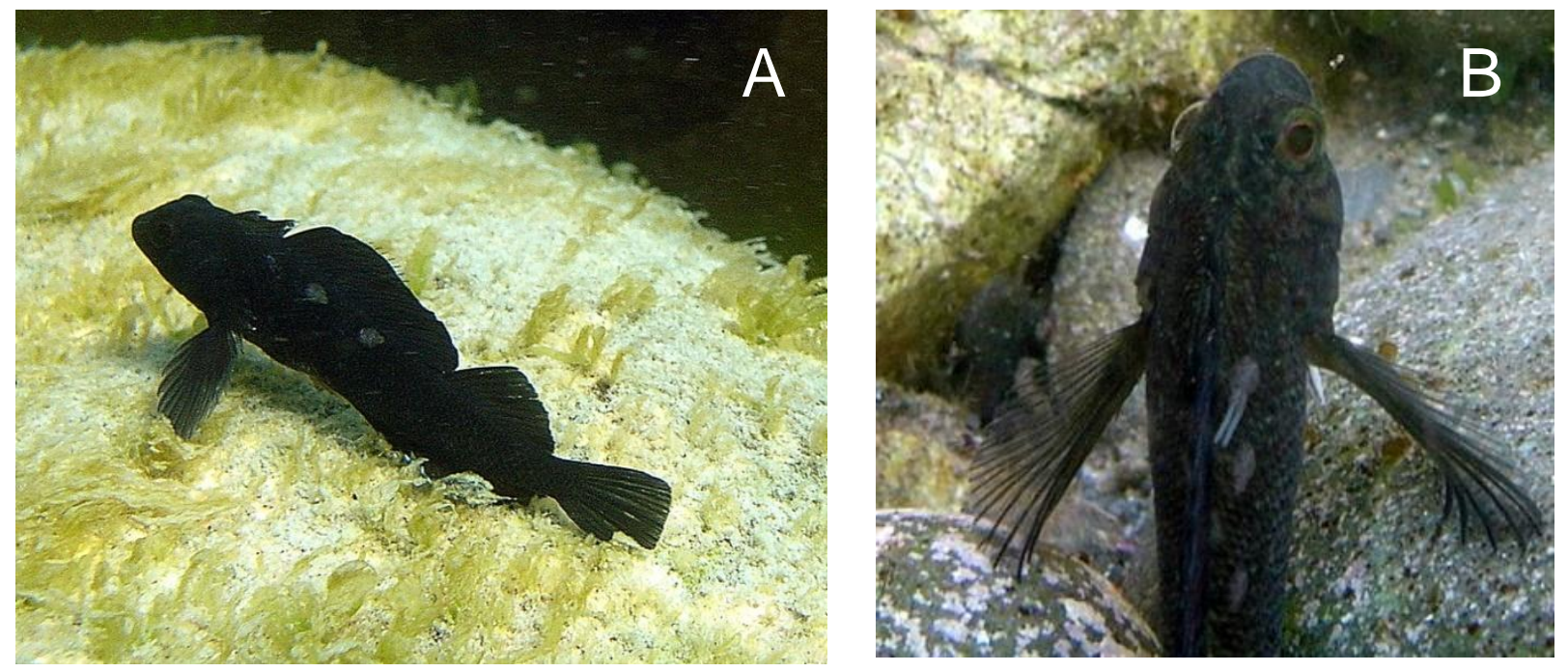

Figure 5.1A Photographs of parasite infections (Caligus buechlerae) on males of the common triplefin (Forsterygion lapillum) B A male common triplefin with three $C$. buechlerae. Uppermost parasite is a female with distinctive egg sacs trailing the body 


\subsubsection{Surveys}

This study was undertaken from 20 - 25 November 2013 on the exposed south coast of the Miramar Peninsula in Wellington, New Zealand (41 $\left.20^{\prime} 40.42^{\prime \prime S}, 174^{\circ} 48^{\prime} 59.77^{\prime \prime E}\right)$. The shallow subtidal ( 0-3 $\mathrm{m}$ depth) of Miramar Peninsula is characterized by a large number of cobble fields, within which I selected $18 \times 1 \mathrm{~m}^{2}$ quadrats spaced at a minimum distance of $5 \mathrm{~m}$ apart. I selected cobble fields of various sizes across the site (based on the presence of triplefins) and then randomly selected an area within the cobble field to place the quadrat. Within each quadrat, an observer on snorkel counted and estimated total length (TL) of all males (identified by their black breeding colouration). Prior to surveys, the observer was trained to estimate fish lengths using a sample of live triplefins with known lengths from the study area. I visually inspected each male in situ for the presence of ectoparasites (which were highly conspicuous, see Fig. 5.1) and when present, recorded their position relative to five distinct body regions for each side (left or right) of the fish - upper or lower head (snout to end of first dorsal fin), upper or lower mid body (start of second dorsal fin to start of third dorsal fin), and tail (start of third dorsal fin to end of caudal fin). The head and mid-body regions were split into upper and lower based on an arbitrary mid-line. Although adult $C$. buechlerae are highly conspicuous, the detectability of smaller immature stages (e.g., chalimus stages) may be lower. Immature stages are likely to be less pathogenic than adults, given that they are immobile and cause only minor localized damage at their attachment site (Johnson et al. 2004); therefore, I chose to focus on how adult $C$. buechlerae may modify the reproductive success of infected males. In addition, as I was primarily interested in the effect of ectoparasites on reproductive success I did not make any attempts to investigate how smaller parasite species (e.g., intestinal tape worms) may have influenced reproductive success in $F$. lapillum.

For each male, I recorded whether it was associated with a nest based on the display of nest guarding behaviour and the presence of eggs. In order to estimate clutch size (the number of eggs within each nest), I briefly overturned the cobble on which the nest was laid and photographed the clutch with a scale bar in situ. Then with the image analysis software Image Pro Plus ${ }^{\circledR}$ (v5.0), I measured the total surface area occupied by the eggs within each photograph by tracing the outline of the eggs, which are laid in a single layer. This technique offers an approximation of clutch size, because the egg layer may not be completely continuous, having 
small empty patches (e.g., due to small areas of bryozoans) or slight differences in egg spacing between nests. Although nests may contain clutches from multiple females, I focused my measurements on clutch size as my goal was to estimate the reproductive output of males (total egg area), not necessarily the number of clutches they received. Four nests were excluded (out of 40) from the analysis because photograph quality was too poor, or the substrate the eggs had been laid on did not allow for the accurate measurement of the surface area occupied by the eggs (e.g., the interior of an empty abalone shell). Triplefin males generally guard one nesting site at a time until all eggs have hatched (Thompson 1986), and in my survey no males were found defending more than one nest, suggesting that during sampling I were likely able to photograph all of the eggs from that paternal male. However, I acknowledge that my method provides a snapshot of reproductive success for individual males and that my measurements may be subject to unknown error caused by temporal variation in success over the breeding season.

\subsubsection{Statistical Analysis}

I used mixed models to examine the factors that: (i) contribute to the likelihood of parasite infection; (ii) influence the probability of a male having a nest; and (iii) affect nest size variation among parental males. For each model, I included a random effect of quadrat ID (1-18) to account for correlations between individual males within the same quadrat. To examine the factors that contribute to the likelihood of parasite infection, I used a generalized linear mixed model (GLMM) with a binomial error distribution. Infection was fit as a binomial response (1infected, 0-no infection) with fixed factors of fish total length (hereafter total length), male density (males quadrat ${ }^{-1}$ ) and an interaction term between total length and male density. Density is often positively correlated with parasite prevalence in many species including reef fish (Finley and Forrester 2003), and I hypothesized that higher male density may lead to greater infection rates.

To assess the probability of a male having a eggs, I used a GLMM with a binomial error distribution. I fit a binomial response for nest ( $1=$ eggs present, $0=$ no eggs) with fixed factors of total length, parasite infection, proportion of infected males (infected males quadrat ${ }^{-1} /$ males $^{2}$ quadrat $^{-1}$, to assess the influence of local infection prevalence on egg presence), and male density. I included several interaction terms to investigate: (i) how parasite infection may influence the importance of body size on reproductive success (parasite infection $\times$ total length); 
(ii) how local infection prevalence modifies the effects of parasite infection on reproductive success (parasite infection $\times$ proportion of infected males); and (iii) how local male density mediates the importance of parasite infection on reproductive success (parasite infection $\times$ male density).

Finally, I fit a linear mixed model to examine the factors influencing clutch size (egg area). I included fixed factors of total length, parasite presence, and nest density (nests quadrat ${ }^{-1}$ ) including an interaction between parasite presence and total length (parasite infection $\times$ total length) to determine if parasite infection influenced the effects of body size on egg area.

For each analysis, I used Akaike Information Criterion (Akaike 1974) with a correction for small sample sizes (AICc values) to rank the complete set of candidate models, including all fixed factors and interactions (6 models for parasite infection, 35 models for egg probability, 10 for egg area). I used parametric bootstrapping to construct confidence intervals for the fixed effects in my top models (simulations=1000). For the binomial models, I used a bias corrected and accelerated method (Efron 1987) for confidence interval selection (due to skewness in the bootstrapped distrubution) and for the linear model I used the percentile method. For statistical significance in both of the binomial models, I used Wald z scores. For the linear mixed model (egg area), I used a likelihood ratio test (LRT) to compare models with and without the main effects and inferred statistical significance if the more complex model had a significantly better fit (Bolker et al. 2009).

All statistical analyses were conducted in the statistical software R (R Core Team 2013). I used the package "Ime4" (Bates et al. 2013) with the function glmer to conduct GLMMs. When investigating the probability of infection and the probability of having eggs, candidate models were generated from global models using the dredge function in the "MuMIn" package (Barton 2013). Bootstrapping was conducted using the bootMer function in the lmer package and confidence intervals were extracted using the boot.ci function in the "boot" package (Canty and Ripley 2013).

\subsection{Results}

I surveyed 124 males $\left(6.8 \pm 2.3\right.$ males $\mathrm{m}^{-2}$, mean \pm stdev $)$ with an average total length of $57 \pm 4 \mathrm{~mm}$. In total, I found 84 C. buechlerae ectoparasites, with $37.9 \%$ of males being infected. 
For infected males, the number of $C$. buechlerae ranged from 1 to 5 individuals male ${ }^{-1}$ with a median of 2 individuals male ${ }^{-1}$. C. buechlerae were found most often on the upper mid-body (63\%), followed by the lower mid-body (29\%), but appeared to be evenly spread on both the left $(55 \%)$ and the right sides $(45 \%)$.

For parasite presence, AICc values indicated that the top model only included fixed factors of total length (Table 5.1). The likelihood of parasite infection significantly increased with body size, with a $73 \%$ increase in the odds of being infected for every $1 \mathrm{~mm}$ increase in length (Fig 2A).

I found that $32 \%$ of males surveyed were defending nests containing eggs. Nest density was variable ranging from to 0 to 7 nests $\mathrm{m}^{-2}$, with an average of $2.2 \pm 2.4$ nests $\mathrm{m}^{-2}$ (mean \pm stdev). The percentage of males that were harbouring at least one ectoparasite was $62.5 \%$ in males that were defending eggs and $26.2 \%$ in males that were not defending eggs. The top model included fixed effects of total length, proportion of infected males and male density, but not parasite infection (Table 5.1). Total length had a significant effect on the probability of having eggs (Table 5.1) with a 49\% increase in the odds of having eggs for every increase of 1 $\mathrm{mm}$ in total length (Fig. 2B). The likelihood of having eggs was correlated with the proportion of infected males (Table 5.1), for every 10\% increase in infection proportion the odds of having a nest increased by $37 \%$. Male density also had a significant effect on the probability of having eggs (Table 5.1), with a 35\% increase in the odds of having eggs for every one added male.

Mean clutch size was $1938 \pm 899 \mathrm{~mm}^{2}$ (mean $\left.\pm \mathrm{stdev}\right)$. AICc values suggested that there was strong support for two separate models (Table 5.1). As the second best model was the null model, I compared the top model (containing total length as a fixed factor) and the null model (no fixed factors) using a likelihood ratio test to determine if the addition of total length significantly improved the model fit. Model fits did not significantly differ between the null model and the top model containing total length $\left(X^{2}=2.699, p=0.100\right)$ indicating that total length did not significantly affect egg area. 
Table 5.1 Top 2 models for each of the two binomial mixed models (parasite prevalence and egg presence) and the linear mixed model (egg area) based on AICc values. Table also includes fixed effect estimates ( $\beta, \log$ odds), bootstrapped confidence intervals (CI, bias corrected and accelerated method for binomial models and percentile method for linear model, simulations=1000), and significance values for each fixed effect (Wald z scores*). Akaike weights (w) are calculated for the two top models only (i.e. weights sum to 1.00) and give an indication of the strength of support for that particular model. In both cases, the top model was used for inference and is highlighted in gray. For the fixed effect of local infection (\% infected), the regression estimate represents a $1 \%$ increase in infection. * The p-value for the effect of total length on nest size was determined using a likelihood ratio test, not a Wald z score (see Results)

\begin{tabular}{|c|c|c|c|c|c|c|c|c|c|}
\hline $\begin{array}{c}\text { Response } \\
\text { variable }\end{array}$ & $\begin{array}{l}\text { Model } \\
\quad \#\end{array}$ & $\mathrm{AICc}$ & $\triangle \mathrm{AICc}$ & $w$ & Fixed Effects & $\beta$ & CI & z score & $\begin{array}{c}\text { p- } \\
\text { value }\end{array}$ \\
\hline \multirow{3}{*}{ Parasite } & 1 & 122.69 & 0 & 0.725 & Total length & 0.549 & $0.361,0.957$ & 4.796 & $<0.001$ \\
\hline & 2 & 124.63 & 1.943 & 0.275 & Total length & 0.548 & $0.360,0.895$ & 4.799 & $<0.001$ \\
\hline & & & & & Male Density & -0.069 & $-0.356,0.285$ & -0.451 & 0.652 \\
\hline \multirow{7}{*}{ Eggs } & 1 & 118.82 & 0 & 0.74 & Total length & 0.396 & $0.249,0.716$ & 4.015 & $<0.001$ \\
\hline & & & & & Male Density & 0.303 & $0.097,0.678$ & 2.326 & 0.02 \\
\hline & & & & & $\%$ Infected & 0.0321 & $0.012,0.0650$ & 2.754 & 0.005 \\
\hline & 2 & 120.91 & 2.09 & 0.26 & Total length & 0.415 & $0.214,0.750$ & 3.632 & $<0.001$ \\
\hline & & & & & Male Density & 0.301 & $0.036,0.599$ & 2.313 & 0.021 \\
\hline & & & & & $\%$ Infected & 0.0344 & $0.009,0.069$ & 2.552 & 0.01 \\
\hline & & & & & Parasite & -0.221 & $-1.431,1.222$ & -0.35 & 0.727 \\
\hline \multirow[t]{2}{*}{ Nest size } & 1 & 597.43 & 0 & 0.52 & Total length & 98.67 & $-32.21,219.9$ & - & $0.100 *$ \\
\hline & 2 & 597.59 & 0.159 & 0.48 & None & - & - & - & - \\
\hline
\end{tabular}



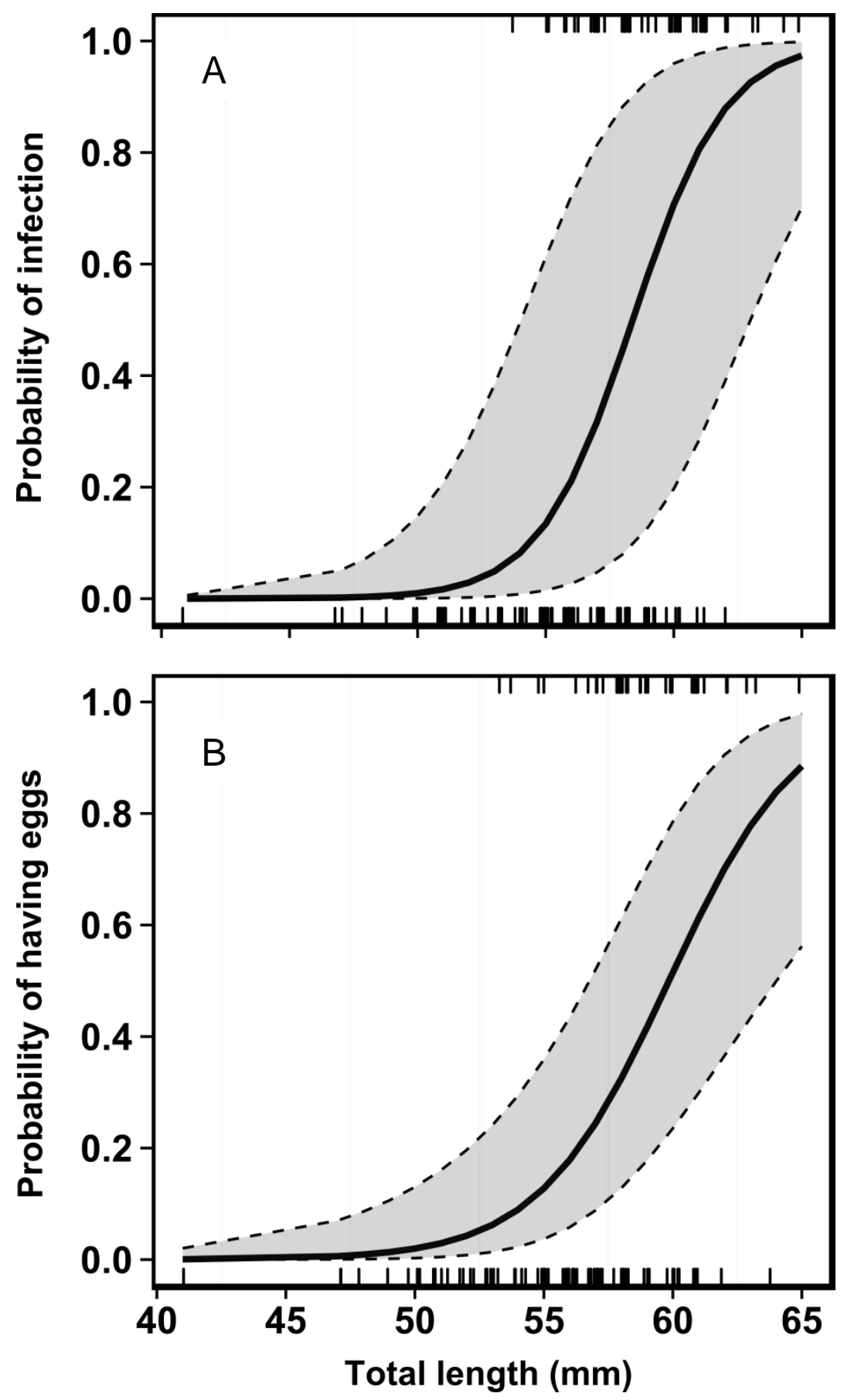

Figure 5.2A The probability of parasite infection based on total length (x-axis) for male adult triplefins surveyed at Moa Point B The probability of males having a nest based on total length (x-axis). To ease visualization, model covariates for the egg model were fixed to their means. Grey areas represent standard error of the model fit including variance from the random effect, but not accounting for uncertainty in estimating the random error variance. Tick marks on the top and bottom axis represent individual fish (infected $=1$, uninfected $=0$ ) and are adjusted by $0.3 \mathrm{x}$ axis increments to indicate point density 


\subsection{Discussion}

I measured the correlation between the presence and area of eggs in a male's nest, ectoparasite infection, and total length to test the hypothesis that parasitised males experience reduced reproductive success. I found that both parasitism and reproductive success (presence of nests) were positively correlated with body size; however, when body size was statistically accounted for, parasite infection had no effect on reproductive success. Although I did observe a significant (and positive) effect of local proportion of infected males on the probability of having eggs, I suspect that this is likely caused by the positive covariation between average male size and the number of infected individuals per quadrat, rather than a positive influence of the parasite on reproductive success. In a closely related species, Forsterygion varium, reproductive success of males was found to be driven by a combination of territory quality and male size (regardless of territory quality), although larger males also occupied the highest quality habitats (Thompson 1986). Larger body size also influences intraspecific competition for preferred nesting habitats in other triplefin species (Wellenreuther et al. 2007b), which suggests that larger size may play an important role in mating success, despite the higher probability of ectoparasite infection.

My results corroborate previous studies on marine reef fish (Barber 2002, Cheney and Côté 2003), suggesting that parasitic effects on male mating success may be weak. However, several factors may explain the absence of parasitic effects on male reproductive success observed here. First, relative to unparasitised females, parasitised females may exhibit reduced selectiveness against parasitized males in fish (Poulin 1994); therefore, the prevalence of parasitic infection among females in my study area, which I did not quantify, may have influenced female mate choice decisions (e.g., Poulin and Vickery 1996, López 1999, Beckers and Wagner 2013). Second, the influence of male size on reproductive success in marine fish species, which was important in my study population, may vary over the breeding season (Oliveira et al. 1999, Borg et al. 2006) and/or with the availability of nesting habitat (Hastings 1992). Consequently, assuming parasites negatively influence female fitness, I expect that the relative importance of ectoparasites in modifying reproductive success may co-vary with selection for large body size across wider temporal and spatial scales. Furthermore, I did not measure the time of initial infection or the infection duration in males, which could have affected 
my ability to detect the influence of parasites on male reproductive success. Alternatively, Caligus buechlerae may have minimal deleterious effects on female/offspring fitness and therefore, selection pressures for females to choose unparasitised males would be negligible (Clayton 1991). If females do not directly select against parasitized males, parasitized males could still experience lowered reproductive success if the parasite directly influenced their courtship behaviour (Barber et al. 2000; Pélabon et al. 2005) or their ability to compete with other males (e.g., Forbes 1991). Currently, the parasitic effects of C. buchalerae are unknown, but even weak parasitic effects on males could modify lifetime reproductive success indirectly by substantially decreasing male growth rates (e.g., Finley and Forrester 2003) and in turn, reducing male size.

Overall, my study indicates the role of parasitism in mediating reproductive success in the common triplefin may be weak, at least when compared to strong selection for larger body size, which is common in both triplefins (Thompson 1986, Wellenreuther et al. 2007b) and other reef fish species (Côté and Hunte 1989, Oliveira et al. 1999, 2000). My study provides the first quantitative assessment of ectoparasite infection rates for a common and widespread species within New Zealand and is among the few studies that have investigated the effect of parasites on reproductive success in reef fish generally. Although my results are similar to those of Barber (2002) and Cheney and Côté (2003), this is an emerging area of research requiring further work to determine the generality of the effects (or lack thereof) of parasite-mediated sexual selection in marine reef fishes. 


\section{Chapter 6 - The effects of natal source on larval starvation resistance in a temperate reef fish}




\subsection{Abstract}

In marine reef fish, isolated source populations may be connected (i.e. have gene flow) via larval dispersal; however, differential larval survival between source populations may influence the degree of population connectivity. I investigated the effects of natal source on larval survival by measuring initial starvation resistance in larvae of the common triplefin $(F$. lapillum) sourced from two distinct natal sources in Wellington, New Zealand. Average survival time was 3.75 days, but survival analysis indicated that starvation resistance did not differ between the two natal sources. Variance components analysis found that the majority of variation was partitioned at the nest level, suggesting that larval survival may be highly variable between individual nests. Mean larval size from each nest was negatively correlated with mean survival time, although this was only apparent in larvae collected from the south coast, which also had a higher variability in initial larval size. My study indicates that variation in larval traits between source populations does exist, but that on average, source populations had equal resistance to starvation. Given the differences between source populations in (i) the relationship between larval quality and larval size (i.e., the absence of size effects in the harbour) and (ii) variance in larval size, there may be the potential for connectivity between populations to be mediated by starvation resistance depending upon the level of food availability. 


\subsection{Introduction}

For marine reef fish, dispersal primarily happens in the pelagic larval stage. During dispersal, larvae sourced from spatially isolated adult subpopulations may intermix and consequently, larval supply to each subpopulation may be composed of larvae from multiple sources (Cowen et al. 2006). Through this exchange of larval propagules, groups of subpopulations may become interconnected (i.e., have gene flow) and function as regional metapopulations (Cowen et al. 2006; Cowen and Sponaugle 2009). The level of connectivity or larval exchange among subpopulations is vital for multiple processes, including population persistence (Cowen and Sponaugle 2009). Connectivity between subpopulations will be regulated by (i) the reproductive rate of each subpopulation (ii) dispersal distances and the degree of larval mixing (which will be affected by the strength and direction of water currents), and (iii) the relative survival of larvae from each subpopulation. Current models of population connectivity in reef fish assume that larval mortality during the pelagic stage is equal among subpopulations (e.g. Cowen et al. 2000; James et al. 2002); however, the validity of this assumption is rarely tested. Larval survival has the potential to vary substantially between sources, especially considering that larval mortality can vary significantly even within subpopulations (Berkeley et al. 2004). The differential survival of larvae among subpopulations may modify the proportional contribution of each subpopulation to overall larval supply, and therefore potentially mediate population connectivity at regional scales.

Mortality in recently hatched larvae can be high (Fortier and Leggett 1985) and food limitation is considered one of the most important contributing factors to mortality during this early stage (Hjort 1914, Cushing 1975). Resistance to starvation may potentially differ between subpopulations; for example, due to environmental heterogeneity between spawning sites. For instance, increased temperature during incubation may reduce starvation resistance by hastening yolk-sac exhaustion (Mendiola et al. 2007) or potentially via increases in embryonic metabolism during incubation (e.g. Bochdansky et al. 2005). Increased incubation temperature may also lead to decreased size-at-hatch (Pepin et al. 1997; Mendiola et al. 2007) and evidence suggests that not only are smaller larvae less resistant to starvation (Miller et al. 1988), but they may also experience higher post-settlement mortality (Vigliola and Meekan 2002; although see Raventos and Macpherson 2005). In addition to environmental variability, starvation resistance may also 
be affected by differences in population structure, such as maternal age or size (Berkeley et al. 2004; Birkeland and Dayton 2005). If larvae from spatially isolated subpopulations differ in their starvation resistance, then early larval survival rates may vary among source populations and in turn could alter connectivity within metapopulations (e.g., Shima et al 2010).

In this paper, I investigate how source population influences larval survival by quantifying initial starvation resistance in a common and widespread New Zealand reef fish, Forsterygion lapillum (the common triplefin). The common triplefin is one of the most common benthic fish in shallow coastal areas around New Zealand (Willis 2001; Wellenruether et al. 2007a; Wellenreuther et al. 2008) and in my study area, is the most abundant larval fish in the water column (Crispin 1998). Females typically lay eggs underneath cobbles within the territory of males (Feary and Clements 2006). Males defend clutches of eggs deposited by multiple females, with larvae hatching after approximately 3 weeks (Francis 2012). Larvae remain in the pelagic stage for a period of 1-3 months (Shima and Swearer 2009a; Kohn and Clements 2011). Adult and juvenile F. lapillum are extremely site-attached (Clements 2003, Shima et al 2013); consequently, dispersal between source populations during the juvenile and adult phases is extremely unlikely. In my study area, elemental otolith signatures suggest that there are likely two main source populations: the Wellington harbour and the exposed south coast (Shima and Swearer 2009, 2010). Temperatures typically differ between the regions, with the exposed south coast $\sim 1-2^{\circ} \mathrm{C}$ cooler than the harbour during the breeding season (Helson et al. 2007). Although source population does not appear to affect post-settlement survival or the larval quality of settlers that survive to settlement (Shima and Swearer 2010), the potential for these two natal sources to differ in early larval survival has not been examined. Here, I aim to determine if larval survival differs between these two natal sources (harbour and south coast) by examining starvation resistance in larvae under controlled laboratory conditions. In addition, I quantify variation in both egg and larval size between the harbour and the south coast to examine how these traits may vary between natal sources. 


\subsection{Methods}

\subsubsection{Collection of larvae}

I conducted a laboratory assay to monitor the survival of individual larvae sourced from within the harbour or the exposed south coast. I collected larvae from around the Miramar Peninsula in Wellington, New Zealand with 2 replicate sites (small bays 50-100m across) within each source population: Wellington harbour -Kau Bay and Mahunga Bay; exposed southern coast - Wahine Park and Moa Point). I ensured replicate sites were similar in exposure and relatively close to one another (100's of meters) and therefore likely to experience similar environmental conditions. All selected sites were in sheltered bays characterized by large areas of substrate covered with cobbles (the most common habitat used by F. lapillum for spawning, Feary and Clements 2006). At each site, I collected F. lapillum eggs from shallow water $(<2 \mathrm{~m}$ deep) on two dates during the spawning season, 16 November and 3 December 2012. I haphazardly searched sites for eggs by inspecting the underside of flat cobbles. I selected nests if larvae had "silver eyes", which indicates larvae are fully developed (Warren 1990) and that hatching is about to occur (Neubauer et al. 2010). From each site, I collected six nests and transported them to the Victoria University Coastal Ecology Laboratory (VUCEL) in individual buckets. Upon arrival at the laboratory, I checked buckets for hatched larvae, and I selected three nests with hatched larvae from each site for experimental trials. I only used larvae that had hatched upon arrival.

From each of the selected nests I haphazardly selected 20 larvae using a plastic pipette; 10 larvae were used for assay trials (described below) and 10 larvae were reserved for measuring larval lengths. I did not directly measure length for larvae that were going to be used in experimental trials because I wanted to avoid stressing larvae, which may have had negative effects on their survival. In two nests (out of 24), I was limited by available larvae, and for these two samples I used all hatched larvae for assay trials and none were available for larval measurements. In two other cases (out of 24), limited larval availability permitted measurements of <5 larvae (after removing the full set for assay trials); therefore, I excluded these nests from the analysis of larval length. In the remaining nests $(n=20)$, I was able to collect at least 7 larvae from each nest for length measurements, with a mean of 9.1 larvae nest $^{-1}$. In total, I measured 182 larvae ( 80 from the harbour, 102 from the south coast) from 20 individual nests ( 9 from the 
harbour, 11 from the south coast). To measure larval length, larvae were euthanized and placed individually on microscope slides, photographed at 10X magnification and measured from the tip of the snout to the end of the caudal fin using Image Pro Plus ${ }^{\circledR}$ (v 5.0).

I was able to measure egg diameter from only $\sim 60 \%$ of the sampled nests $(n=14$ for harbor and south coast), because in many instances all of the eggs had hatched before I could measure them. To measure egg size, I removed each rock from its container and positioned it with the side containing the nest facing upwards. I randomly selected 10 eggs from each nest, gently transferred them onto a glass slide using forceps, and photographed eggs at $8 \mathrm{X}$ magnification under a dissecting scope. Then using the photographs, I haphazardly measured two diameters at perpendicular angles for each egg with Image Pro Plus ${ }^{\circledR}$ (v. 5.0) and then averaged both measurements to obtain the overall egg diameter. Because the common triplefin is a polygamous breeder (Warren 1990), selected eggs may have been laid by multiple females.

\subsubsection{Assay design}

I immediately placed larvae that were selected for assay trials into small $(5 \mathrm{~cm}$ in height) round containers (1 larva container ${ }^{-1}$ ) containing $100 \mathrm{~mL}$ of seawater which had been filtered (1 $\mu \mathrm{m})$ and autoclaved in an effort to remove any available food items. The number of larvae from each nest varied $\left(8.6 \pm 2.1\right.$ larvae nest $^{-1}$, mean \pm SD) because a few nests had fewer than 10 hatched larvae available and some mortality occurred during transfer from buckets. Each container was wrapped in black tape to reduce mortality associated with "walling" behaviour (repeated collisions with the side of the container, Cobcroft and Battaglene 2009). Each container was randomly positioned on a $45 \mathrm{~cm}$ x $240 \mathrm{~cm}$ sea table using a grid and the sea table was flooded with approximately $2 \mathrm{~cm}$ of flow-through seawater to maintain ambient sea temperature $\left(15.0^{\circ} \mathrm{C} \pm 0.73\right.$, mean $\left.\pm \mathrm{SD}\right)$.

After larval transfer to containers, I monitored larval mortality for an initial 12 hour period prior to the assay and considered any larvae that died during this period to be a result of handling mortality (these deaths were not included in the analysis). I then measured larval survival every 12 hours over the entire period of study until no individuals remained alive ( $\sim \mathrm{d}$ duration). During the trials, each individual container had a 50\% (50 mL) water change every 24 hours. The assay was repeated twice, starting on 16 November $(n=97)$ and 3 December $(n=111)$. 
Any death that was not caused by starvation (e.g. accidental mortality during water changes, 16 Nov assay - 4 larvae, 3 Dec assay - 5 larvae) was recorded as right censored (i.e. mortality due to starvation had not yet occurred, but it is known that the individual had survived up to that point). Survival analysis includes these observations and accounts for their censoring in the statistical analysis.

\subsubsection{Statistical Analysis}

I used a Cox proportional hazards mixed model to assess the fixed effects of (i) source population (harbour or south coast) and (ii) sample date on larval survival. I also included an interaction term between sample date and location to determine if location effects were consistent over time. Cox proportional hazards models determine a standard probability of mortality per time period and then estimate how covariates (e.g., source population) alter this probability. Using a mixed model, I included a random effect of nest ID (1-24) to account for correlations between larvae sampled from the same nest. In addition, I included a random factor for collection site to account for variation between replicate sites. To test for the significance of the interaction term, I conducted an analysis of deviance and compared the deviance between models with and without the interaction term using a $X^{2}$ test. For the selected model, I made inferences on fixed effects using Wald z scores.

To investigate how the variance in survival was partitioned among temporal and spatial factors, I conducted a variance components analysis. I refit the Cox proportional hazards model with each of the hierarchical factors as nested random effects (i.e., sample/source population/collection site/nest). I then extracted the percentage of variance partitioned among sampling dates, source populations, collection sites, and nests.

I tested for differences in larval and egg size between natal sources using two linear mixed models with larval length and egg size as response variables respectively. As before, I fit a random effect of nest ID and collection site. For the larval length model, an F-test indicated that variances between locations were not homoscedastic $\left(\mathrm{F}_{83,101}=0.5483, \mathrm{p}=0.005\right)$; therefore, $\mathrm{I}$ used a weighted variance structure in the linear mixed model, which accounts for different variances between fixed factors. For both larval size and egg size, I tested the significance of the interaction term using a likelihood ratio test. 
I investigated the relationship between survival time and larval length by performing a regression using average survival (days) and mean length for each nest. For this analysis, I excluded any censored data points when calculating the average survival time ( $\mathrm{n}=9$ larvae). To test the correlation between average survival time and average larval size I used a Deming regression, which accounts for variance in both the $\mathrm{y}$-axis and $\mathrm{x}$-axis. The Deming regression uses a variance ratio $\left(\sigma^{2} \mathrm{y}\right.$ variable $/ \sigma^{2} \mathrm{x}$ variable) to fit a regression line through the data points that minimizes the perpendicular distance from the data to the regression line, rather than the vertical distance on the y axis, as in a linear regression. Preliminary analysis indicated that the effects of average larval size differed between source populations, so I analyzed source populations separately. For the Deming regression, I used a variance ratio by calculating the mean variance across all nests for both survival time (harbour $\sigma^{2}=2.94$, south coast $\sigma^{2}=2.69$ ) and larval length (harbour $\sigma^{2}=0.033$, south coast $\sigma^{2}=0.028$ ). Bootstrapped $95 \%$ confidence intervals on the regression slope were calculated using the percentile method (simulations $=1000$ ) and were used to make inferences on whether or not the slope significantly overlapped with zero. In order to determine how sensitive the regression analysis was to changes in the variance ratio (which I based on the mean variance for the $\mathrm{y}$ and $\mathrm{x}$-axis respectively), I also conducted a sensitivity analysis (details in Appendix C).

All statistical analyses were completed in the statistical software R (R Core Team 2013). For the Cox proportional hazards mixed model I used the function coxme in the package "coxme" (Therneau 2012). For linear mixed models (egg size and larval size) I used the function lme in the package "nlme" (Pinheiro et al. 2013). To perform the Deming regression I used the Deming function in the package "MethComp" (Bendix et al. 2013). 


\subsection{Results}

I followed the survival of 207 larval F. lapillum originating from 24 individual egg clutches. Mean survival time for post-hatch larvae was $3.75 \pm 0.12$ days (mean $\pm \mathrm{SE}$ ), with a maximum survival time of 8 days. The analysis of deviance indicated that the interaction term between sample date and source population was not significant $\left(X^{2}=1.421, p=0.233\right)$; therefore, I fit only sample date and source population as fixed effects. Hazard ratios for larvae originating from the south coast were not significantly different from larvae originating within the harbour (hazard ratio $=0.0806$, Wald $\mathrm{Z}$ score $=0.37, \mathrm{p}=0.71$, Fig. 6.1), nor did hazard ratios for larvae sampled during the second sampling date differ significantly from the first sampling date (hazard ratio $=0.205$, Wald $\mathrm{z}$ score $=0.93, \mathrm{p}=0.35$ ). Variance component analysis indicated that $75 \%$ of the variance was at the level of nest, with $24 \%$ of the variance at the site level and $<1 \%$ partitioned at the level of source population. 


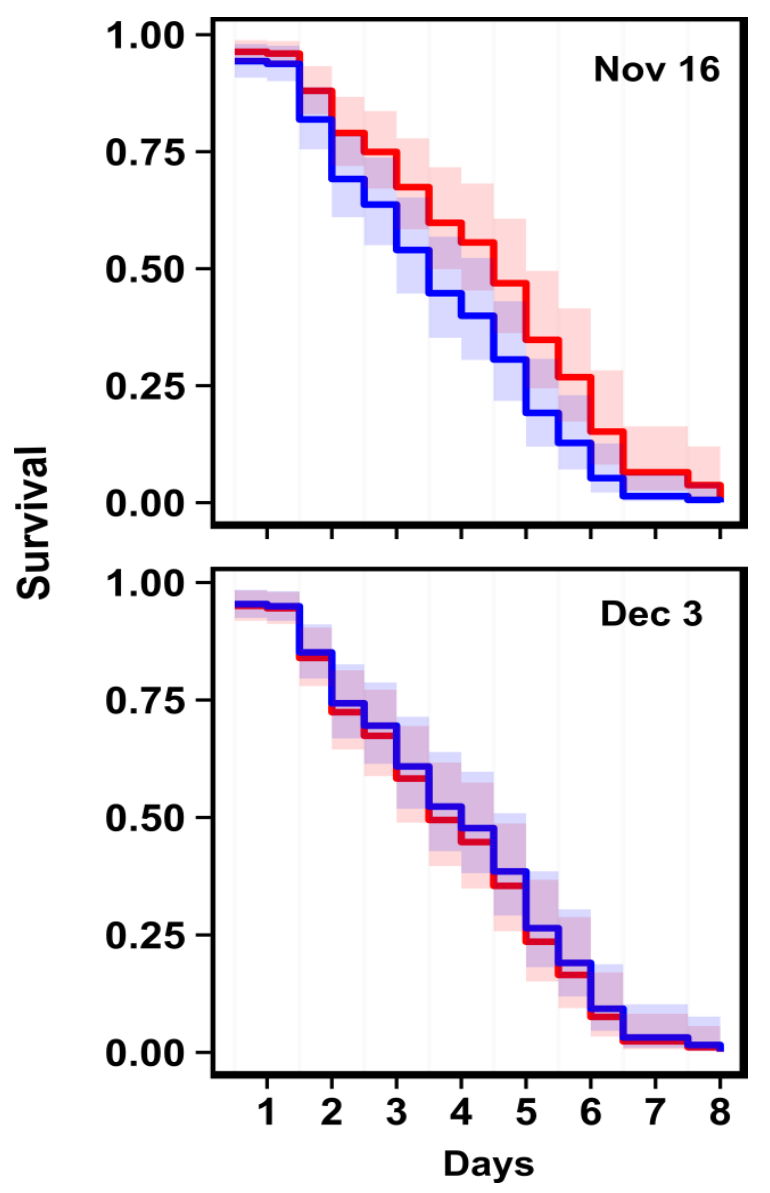

Figure 6.1 Kaplein-Meier survival curves showing the survival rate over time between the harbour (red line) and the south coast (blue line) for two collection dates. 95\% confidence intervals are based on fixed effects only (i.e., only variation within locations) and are shaded around the lines for both the harbour (red shading) and the south coast (blue shading). 
The interaction term between sampling date and source population was not significant for models that explored sources of variation in egg size (likelihood ratio $=0.608, \mathrm{p}=0.435$ ) or larval size (likelihood ratio=1.111, $\mathrm{p}=0.292$ ). Sampling date did not significantly affect egg size $(\beta=0.198, t-$ value $=1.987, \mathrm{df}=1,24, \mathrm{p}$ value $=0.289)$ or larval size $(\beta=-0.007, \mathrm{t}-\mathrm{value}=-0.082$, $\mathrm{df}=18, \mathrm{p}=0.936$ ). Egg size appeared to be larger on the south coast (Fig. 6.2A), although this was marginally non-significant $(\beta=0.034$, $\mathrm{t}$-value $=1.987, \mathrm{df}=24, \mathrm{p}=0.059)$. Larvae from the south coast were significantly larger than those that hatched from within the harbour $(\beta=0.231$, $\mathrm{t}$-value $=2.368, \mathrm{df}=1,18, \mathrm{p}=0.0294$, Fig 6.2B). Mean survival time was unaffected by increasing mean larval size in the harbour (Deming $\beta=-0.423$, bootstrapped 95\% CI $=[-43.065,69.499]$, Fig. 6.3); however, mean survival time significantly decreased with mean larval size on the south coast (Deming $\beta=-3.938$, bootstrapped 95\% CI $=[-8.727,-0.999]$, Fig. 6.3). To illustrate the potential effects this might have on larval contribution from each subpopulation, I ran a simple simulation informed by the results of the Deming regression (Appendix D). 

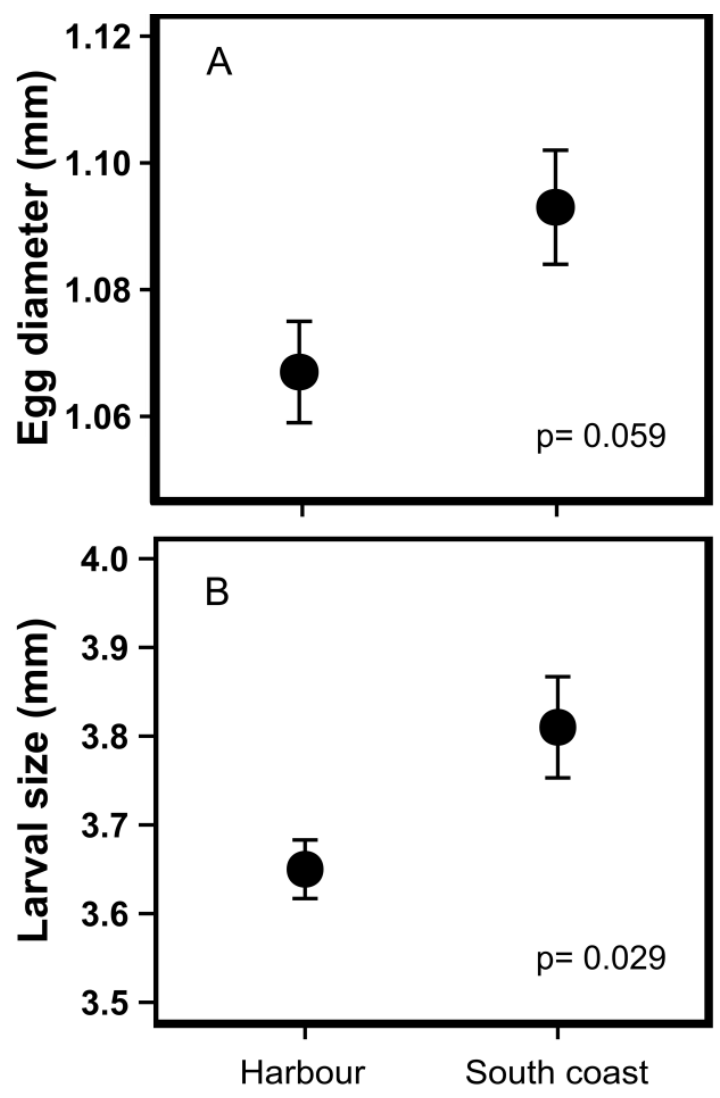

Figure 6.2A Mean egg diameter \pm SE and B Mean larval size \pm SE. For both egg size and larval size, I took the average value for each nest before calculating the confidence intervals, to avoid the effects of pseudoreplication (e.g. multiple larvae from a single nest) on the calculation. However, p-values are calculated from the linear mixed model, which accounted for the correlations between nests using a random factor of nest. Note that egg measurements and larval sizes were not taken from the same nests, and therefore are not directly comparable (i.e., I am unable to conclude that larger larvae on the south coast originated from larger eggs). 


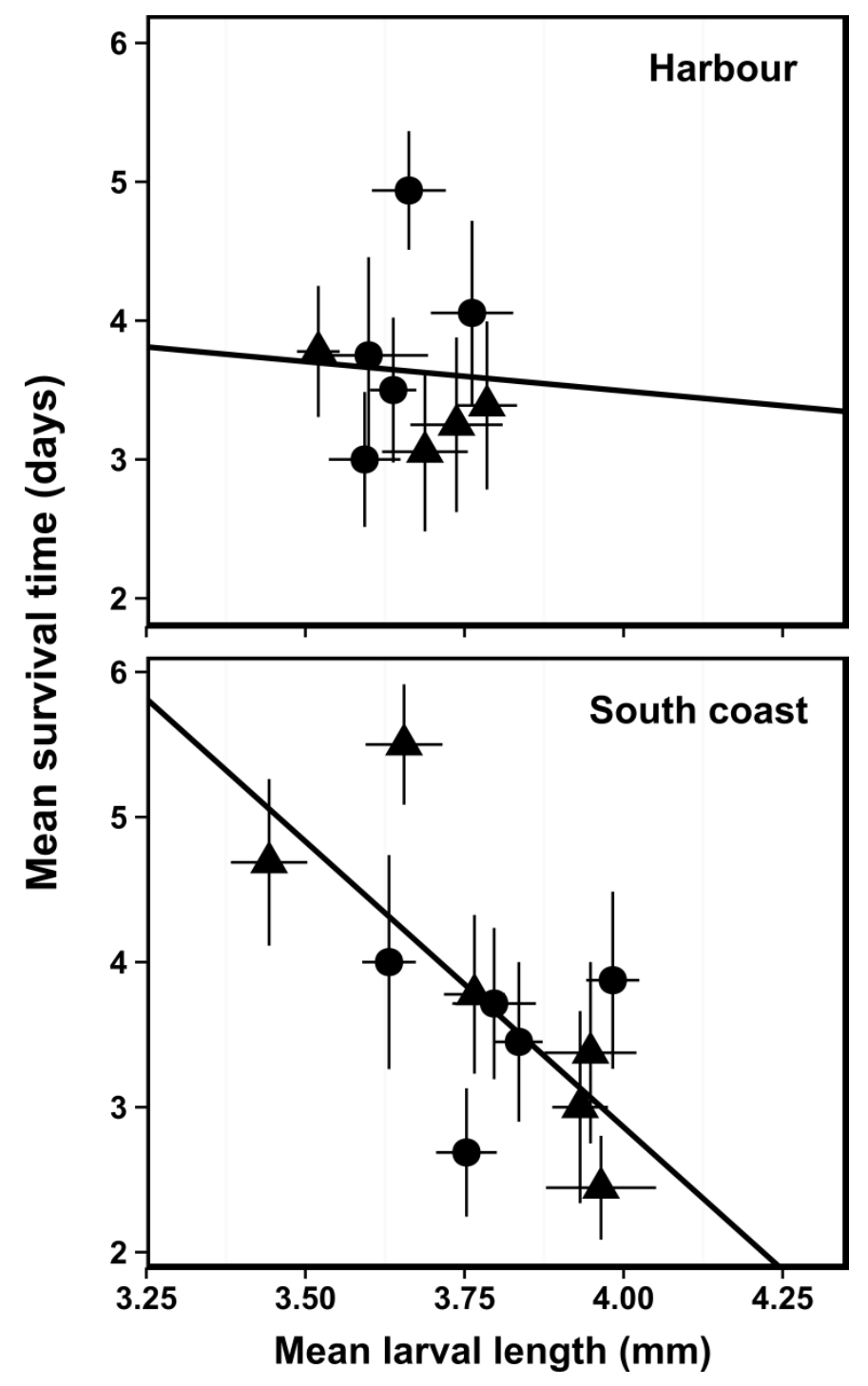

Figure 6.3 Deming regressions between mean survival time and mean larval length among nests collected from two different source locations (harbour and south coast). Each point represents one nest and error bars represent standard errors for both mean survival time (vertical) and mean larval length (horizontal). The regression line for the harbour is not significantly different from zero (Deming $\beta=-0.423$, bootstrapped $95 \% \mathrm{CI}=[-43.065,69.499]$ ), but it is for the south coast (Deming $\beta=-3.938$, bootstrapped 95\% CI $=[-8.727,-0.999]$ ). In each panel, shapes represent collection sites within source populations: harbour (-Kau Bay, $\mathbf{\Delta}$ - Mahunga Bay); south coast (- Moa Point, $\boldsymbol{\Delta}$ - Wahine Point). 


\subsection{Discussion}

In this study, I measured starvation resistance in post-hatch larvae of the common triplefin to investigate larval mortality patterns between source populations. Survival rates between sub-populations did not differ on average; however, larval size and the influence of larval size on starvation resistance did vary among populations. South coast larvae had a larger size-at-hatch and a greater range of sizes (i.e., variance) than the harbour. Data from Chapter 3, indicate that adult females were larger on the south coast (south coast $=57.8 \pm 0.47$, mean $\pm \mathrm{SE}$, harbour $=52.0 \pm 0.59$, one way ANOVA, $\mathrm{F}_{1,110}=58.32$, $\mathrm{p}<0.001$ ), which could have potentially been driven by faster growth rates on the south coast (Chapter 4). Larger females may produce larger eggs and larvae (Chambers and Leggett 1996), potentially explaining size differences between source populations. Starvation resistance appeared to be size-dependent on the south coast, where nests with larger larvae had significantly higher mortality rates; however, this was not observed in the smaller larvae originating from the harbour. These results suggest that larger size-at-hatch, which appears to be associated with lower survival at settlement in the common triplefin (Shima and Swearer 2009), may also reduce starvation resistance for larvae sourced from the south coast. The combination of (i) larger size-at-hatch, (ii) increased variance in larval size, and (iii) the negative relationship between starvation resistance and larval size on the south coast may have important consequences for the relative contribution of south coast and harbour larvae to the larval pool under certain conditions. For example, when food availability is low, larger larvae from the south coast may perish faster than smaller harbour larvae; however, if food does not become available until 7 or 8 days after hatching, then smaller larvae from the south coast survive longer than harbour larvae. This suggests that variability in food supply may not only affect overall recruitment strength (e.g., Platt et al. 2003), but also potentially the degree of larval exchange and consequent gene flow between local populations. Overall, larval survival varied little between spatial factors (site and location), but was highly variable among nests, indicating that reproductive output in males may be strongly modified by larval survival among nests. If starvation resistance contributes heavily to larval mortality patterns, it could have important effects for larval exchange among subpopulations.

Several factors in the design of my study have important consequences for the generality of the results. For instance, I did not monitor or control for incubation temperature in the nest, 
which may be highly variable between source populations, and could have influenced starvation resistance (Mendiola et al. 2007). Furthermore, I did not test for the influence of temperature on starvation resistance in my assay; however, in the field survival patterns may be altered if larvae hatched from different sub-populations experience different temperatures before larvae mix in the open water. In addition, by measuring starvation resistance in post-hatch larvae with no food supplement, I did not quantify a "point of no return", the point where larvae are unable to recover even if they encounter food because they are not strong enough to feed (e.g., Rana 1985). If the point of no return varies considerably between sub-populations (possibly due to differences in larval size), it may modify the patterns of mortality seen here. Although I found that larger larvae on the south coast were less resistant to starvation, they may also be able to access greater particle sizes earlier in larval development, due to increased gape size (Ware 1975), which may mitigate decreases in starvation resistance if food supplies or larger prey items are available. For individuals who survive initial mortality, larval size may also confer benefits at settlement (e.g., Vigliola and Meekan 2002) which could modify survival patterns beyond starvation resistance and dictate the relative importance of post-hatch vs. post-settlement processes.

Here, I only investigated one period during the breeding season; however, early larval survival may vary seasonally. For example, egg size from within both source populations (harbour and south coast) is smaller at the end of the spawning season (Chapter 4), which is a common trait in many fish species (Chambers and Leggett 1996). Given the strong relationship between egg size and larval size, this suggests that larval sizes may be larger earlier on in the season and therefore, may be less resistant to starvation resistance (at least on the south coast). Reduced starvation resistance in larvae hatched early in the season may explain why food supplies earlier on in the breeding season have a particularly important effect on recruitment in some species (e.g., Platt 2003). However, females may trade off larval size with other larval traits, such as yolk sac size over the breeding season (Semmens and Swearer 2012) and therefore the influence of larval size on starvation resistance may vary through the breeding season. If larval size affects starvation resistance over the breeding season as well as differentially between source populations (as observed here), then seasonal patterns in population connectivity may exist and should be investigated. 
The results of this study indicate that variation in larval traits between source populations does exist, but that on average, source populations had equal resistance to starvation. However, analysis suggests that size-at-hatch (which I could not directly account for in the survival analysis) may have had negative effects on starvation resistance on the south coast. Furthermore, given the differences between sub-populations in (i) the relationship between larval mortality and larval size (i.e., the absence of a negative relationship between size and survival in the harbour) and (ii) overall variation in larval size, the potential for connectivity between populations to be mediated by starvation resistance may be dependent upon of the level of food availability. 


\section{Chapter 7 - Contributions and discussion}


The main goal of this thesis was to gain an understanding of the key factors that influence the processes of recruitment, growth, and reproduction of a marine reef fish. In order to accomplish this, I examined novel research questions (listed in Chapter 1) involving multiple life stages (larval, juvenile, and adult). The research in the preceding chapters has provided valuable insights into multiple ecological processes in the common triplefin, which may have broad implications for reef fish in general. Overall, the major and significant findings of this thesis are:

(1) In Chapter 2, I establish that the distribution of adults and juveniles is non-random and follows a curvilinear pattern, with a negative relationship between juvenile densities and adult densities when adult densities are high.

(2) In Chapter 3, I validate the use of intercirculus spacing to estimate previous growth rates in individuals of the common triplefin

(3) In Chapter 4, I show that early growth rates in individual males can be negatively correlated with later reproductive output

(4) In Chapter 5, I show that parasite infection co-varies with body size, but that parasites may have weak effects on modifying reproductive output among males

(5) Finally, in Chapter 6, I find that subpopulations differ in their larval characteristics and survival patterns and suggest that under certain criteria, these differences may influence population connectivity

In this final chapter, I attempt to highlight how my results collectively extend our knowledge of reef fish ecology and offer insight into factors that may contribute to fluctuations in populations over time. 


\subsection{Patterns of co-occurrence among adults and juveniles}

The influence of older age classes on determining the distribution and abundance of recently transitioned larvae is becoming more appreciated (Wilson and Osenberg 2002; Webster 2004; Wilson 2005; Samhouri et al. 2009), and my results add to the growing literature that suggests adult distributions may play a vital role in determining spatial patterns of recruitment. Accounting for the role of resident adults on spatial variability in recruitment may refine our ability to predict recruitment variation over time. Increased growth rates in settlers immediately after their settlement transition may be able to dampen the negative effects of adults by reducing vulnerability to cannibalism; however, increased growth may also have subtle, but negative, influences on later reproductive output. The observed decline in adult density as the recruitment season progresses could have important consequences for the timing of spawning and the relative importance of intracohort vs intercohort processes in shaping juvenile distribution patterns. For example, larvae settling early in the season may encounter higher adult densities, but lower settler densities and vice versa for settlers arriving late in the season.

Beyond the settlement site, negative relationships between age classes may have wider effects on metapopulation dynamics if spawning stock is coupled with local larval supply. Research indicates that marine populations may be relatively closed, with larval dispersal being more limited than previously presumed (Cowen 2000; Swearer et al. 1999). Local retention of larvae near their natal source may be high, resulting in self-recruitment (Jones et al. 1999; Swearer and Shima 2002; Almany et al. 2007). In F. lapillum, major recruitment events both within the harbour and on the south coast can be self-recruiting (Swearer and Shima 2010), which suggests larval supply may be linked tightly to local production. However, in subpopulations that have high larval retention, the presence of negative age-class interactions suggest that spawning stock may limit recruitment success via negative density dependent feedbacks on settling larvae.

\subsection{Measuring ecologically relevant growth using fish scales}

Estimating growth trajectories in individuals longitudinally throughout ontogeny in juvenile and adult reef fish has traditionally been difficult. Scale-derived growth estimates may 
provide a non-lethal and simple way to answer critical ecological questions about juvenile and adult life histories in reef fish that have traditionally been impractical to investigate in the field. For example, intercirculus spacing could be used to investigate the detrimental effects of parasites, like Caligus buechlerae, on individual growth and potentially reveal indirect effects of parasites on reproduction by limiting male body size. Intercirculus spacing could also help investigate whether resident adults at the settlement site negatively influence the growth of juveniles. Intercirculus spacing can provide an invaluable tool to further explore the role of a wide array of questions, including how different environmental (temperature, exposure, etc.) or biological (e.g., competition) factors influence growth rates. Alternatively, intercirculus spacing could be used to evaluate the role of growth in vital ecological processes such as reproductive success (e.g., the effects of growth on male reproductive success in Chapter 4).

\subsection{Male reproductive success is influenced by previous growth}

Using scale-derived growth rates, I was able to investigate the influence of growth throughout ontogeny, and found that faster growth rates immediately after settlement were associated with lower fecundity, regardless of size class. My study provides one of the first estimates of previous growth effects on reproductive output for reef fish. Increased growth during the post-settlement stage can reduce settler mortality (Islam et al. 2010); however, my results indicate it may also reduce fecundity. These results, although relatively modest in their effects, suggest that post-settlement life stages in marine reef fish are not fully autonomous, but that experiences during juvenile development may influence later life histories. The potential negative relationship between reproductive output and fast juvenile growth is especially intriguing given that faster growth should increase male size, which appears to be extremely important for obtaining mating opportunities in F. lapillum (Chapter 5).

\subsection{Parasitic effects on reproductive success}

While evidence from terrestrial and freshwater systems indicates that parasitism can modify individual variation in reproductive success among males; however, despite widespread infection, parasites did not affect reproductive success in F. lapillum. The positive covariation between body size, infection rate, and reproductive success broaches intriguing questions such as 
how do ectoparasites influence reproductive success in populations where body size is under weaker sexual selection? Sexual selection for larger body size in fish often occurs when larger males are able to monopolize mating resources (e.g., Magnhagen and Kvarnemo 1989); however, in areas where high quality oviposition sites are plentiful, the influence of larger body size may be reduced, and thus females may potentially be more selective against parasitism. A rigorous test of this hypothesis might include experiments manipulating nest density to evaluate how selection for size and parasitism vary under differing conditions of nest availability.

\subsection{Relationships between starvation resistance and larval size may cause source populations to differ in larval mortality}

Although starvation resistance in the larvae of the common triplefin did not differ between source populations; significant differences in both the response of starvation resistance to larval size and larval size among populations may have the potential to mediate larval survival. High variation in larval mortality between nests also suggests that measured patterns of reproductive success (i.e., egg number male $^{-1}$ ) may be may greatly modified after hatching by differences in starvation mortality. Furthermore, these results indicate that our current models of larval dispersal may be ignoring potentially important variation in larval mortality between spawning locations that under certain conditions, may affect the relative connectivity of populations. Given the logistical, practical, and feasibility issues associated with accurately determining larval survival rates and variation in dispersal patterns over time, it begs the question, how can we account for variability in larval survival among natal sources and how important is this to population connectivity? Techniques that can estimate natal sources for individual fish such as geochemical signatures are becoming more refined (White et al. 2008), and these methods can determine realized population connectivity between sources, rather than estimating connectivity through dispersal models. Comparisons between model-based predictions and realized connectivity can help uncover how unaccounted biological parameters (e.g., larval mortality) influence dispersal models and their applicability for modeling population connectivity (Treml et al. 2008). Although the relative importance of larval quality between source populations and its effects on population connectivity are poorly understood, we should at least acknowledge how that variation in larval mortality may influence the precision of larval dispersal models (e.g., Siegel et al. 2003). 


\subsection{Concluding remarks}

Overall, this thesis highlights key linkages between life stages and processes through the entire life cycle of a marine reef fish. Results here emphasize that a multi-stage approach can reveal connections within an individual's life cycle (e.g., juvenile growth on reproduction, source population on later larval mortality) or between life stages (e.g., age class interactions) that may be shaping population dynamics. Taken as a whole, the results from this thesis have general importance for the study of reef fish, by identifying key factors including conspecific density, early post-settlement growth, and source population identity that may influence population dynamics through time. 
Appendix A- Description of model
averaging 


\section{Model Averaging}

Model averaging estimates model parameters across several models by averaging weighted parameter estimates from a set of top models based on the likelihood of each model being the best-fit model (determined by AICc weights). Model averaging accounts for variation in parameter estimates based on model selection uncertainty (i.e., the probability of not choosing the best fitting model in the candidate set), which may have important effects on the inferences that can be made from ecological studies (Osenberg et al. 2002). In addition, it also allows inferences to be made from multiple models that may be equally supported (Grueber et al. 2011).

First, all potential models are ranked according to information theoretic (IT) criterion (AICc) and the relative differences from top model (i.e., the lowest AICc score) are calculated $(\triangle \mathrm{AICc}=\mathrm{AICc}-\mathrm{AICc}$ of the top model $)$. All models with a $\triangle \mathrm{AICc}<2$ are selected for model averaging. Among the selected models, the relative support for each model is determined by its Akaike weight, which is then used to complete a weighted average of model parameters using all models. In this way, models which have strong support (higher Akaike weights) have a larger influence on the averaged parameter estimates than models with weak support (lower Akaike weights). If an individual parameter is missing from one or more models in the selected model set, it is given a value of zero for that model (zero method), which is included in the weighted average of the model parameter. Wald Z-scores test for statistical significance of the averaged parameters by calculating a test statistic and an associated p-value by scaling parameter estimates by their standard errors, which can alleviate problems associated with the uncertainty in estimating the effective degrees of freedom utilized by random effects (Bolker et al. 2009). 


\section{Appendix B - Determination of scale size at formation}




\section{Methods for determining scale size at formation}

In order to determine (i) when scale formation occurs and (ii) the approximate total length of the fish at the beginning of the my measurements for intercirculus spacing (i.e., total length at the first post-settlement circuli) I conducted a small survey of pre-settlement fish. As part of another ongoing experiment, light traps (traps which attract and capture phototaxic larvae using bright fluorescent lights) were deployed in Kau Bay, Wellington (within the harbour) in the summer of 2012. From one of these traps I took a sample of 27 fish. For each of these fish, I recorded its total length, whether or not scales had formed behind the pectoral fin (yes or no), and if scales had formed, I measured scale length and number of circuli.

\section{Statistical analysis}

To determine the approximate size of the fish at scale formation I performed a logistic regression with total length as a predictor variable and the presence of scales $(1-$ yes, $0-$ no) as a response. For fish that had scales, I tested the relationship between circuli number and scale size using a linear regression.

\section{Results}

Mean fish size in the sample was $25.5 \pm 0.20 \mathrm{~mm}$ (mean $\pm \mathrm{SE}$ ) and total lengths ranged between $23.99 \mathrm{~mm}-28.78 \mathrm{~mm}$. Out of 27 fish, 12 had formed scales behind the pectoral fin with an average scale size of $323.5 \pm 7.7 \mu \mathrm{m}$. The probability of scale formation significantly increased with size (odds ratio $=1.43$, Wald $\mathrm{z}=2.232, \mathrm{p}=0.0256$, Fig. B1). Among fish that had formed scales, average circuli number was $4.75 \pm 0.25$ circuli and circuli number ranged from 3 circuli - 6 circuli within the sample. Total length was significantly correlated with number of circuli ( $\beta=0.992, r=0.702, d f=1,10, t=3.118, p=0.011$, Fig. B2A). Upon further examination, one point in the dataset appeared to be highly influential (Cook's distance $=2.11$ ) and therefore I re-ran the analysis after removing this point. Again, total length was significantly correlated with circuli number $(\beta=1.58, r=0.866, t=5.194, p<0.001$, Fig.B2B). Estimated total lengths from the regression model including standard errors and prediction (tolerance) intervals are given in Table B1. 


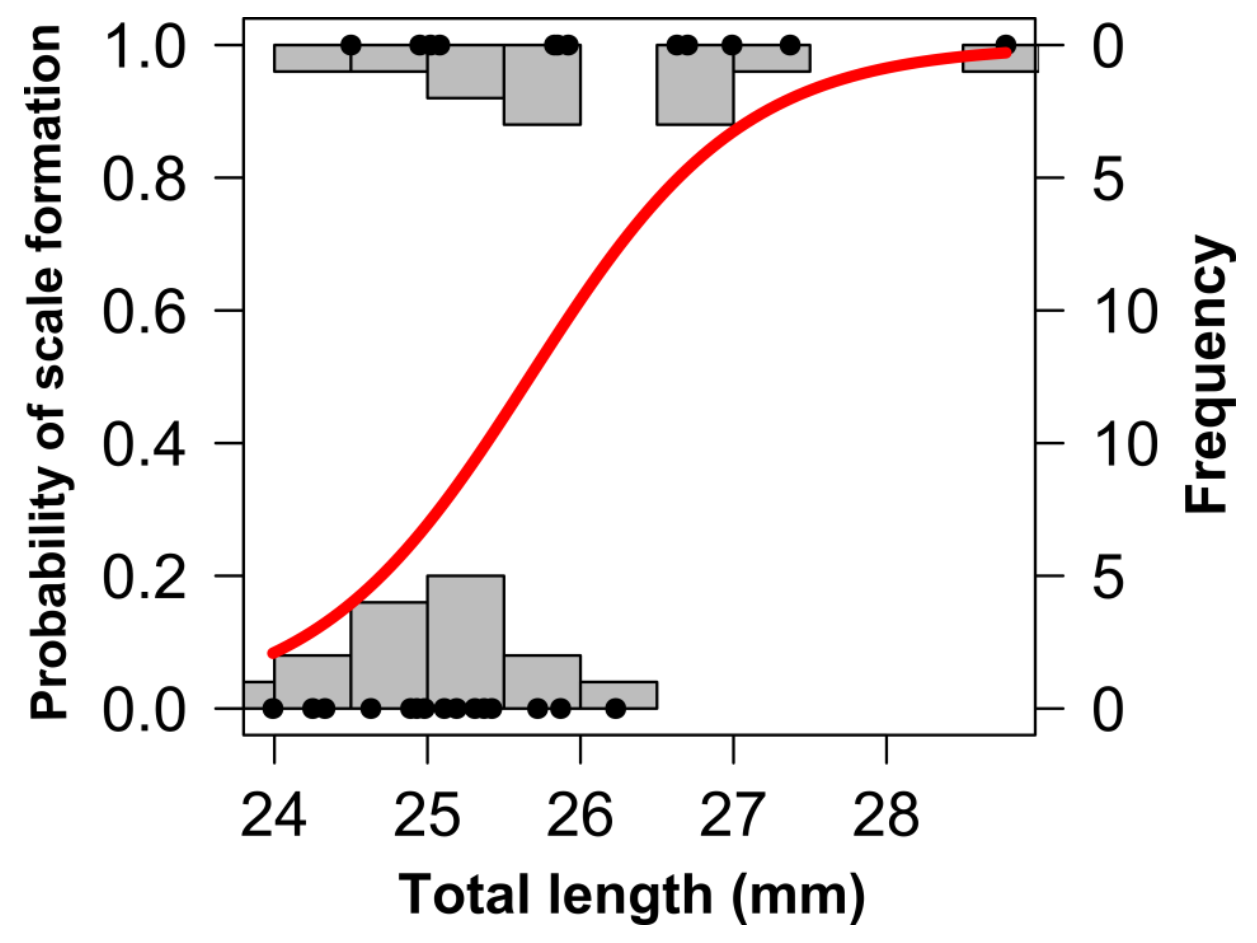

Figure B 1. A logistical regression showing the probability of squamation (initial scale formation) based on fish length. Histograms on the upper and lower axes show the frequency of observations. 


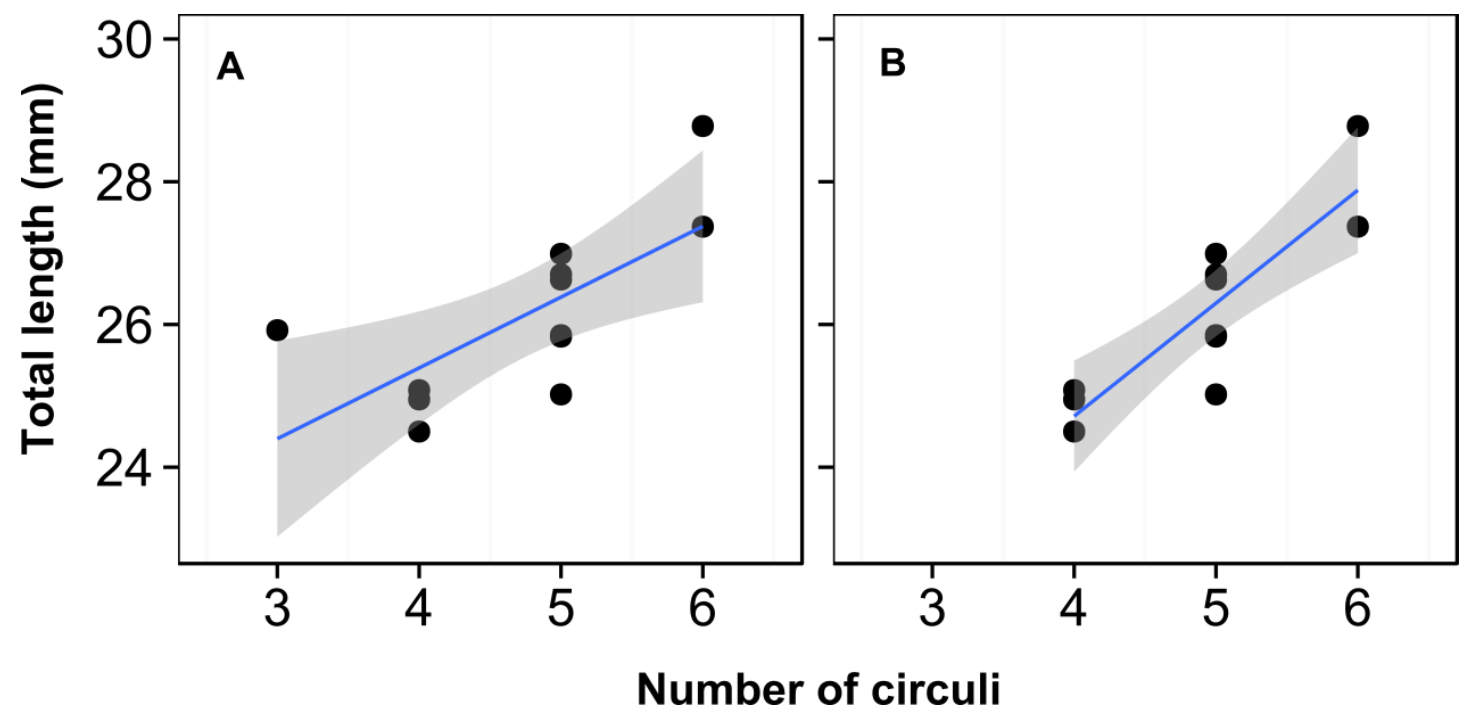

Figure B2. The relationship between total length and number of circuli for fish that had formed scales $(n=10)$. Full data set shown in panel A model with removed outlier shown in panel B. Grey band represents confidence intervals for the regression fit. 
Table B1. Predicted sizes from linear model between total length and circuli number including standard error of the predictions and prediction (tolerance intervals) on the predicted size.

\begin{tabular}{ccccc}
\hline Circuli number & Predicted size $(\mathrm{mm})$ & SE & Lower & Upper \\
\hline \hline 4 & 24.71 & 0.34 & 23.00 & 26.43 \\
5 & 26.29 & 0.21 & 24.70 & 27.89 \\
6 & 27.88 & 0.34 & 26.12 & 29.65 \\
\hline
\end{tabular}

\section{Discussion}

I found no individuals with only 1 or 2 circuli formed, suggesting that either these circuli are formed as part of the initial scale or they growth quickly afterwards. As none of these presettlement larvae had formed more than six circuli, I decided to measure scales collected in Chapter 4 from the sixth circuli onwards, because (i) I wanted to focus on the influence of postsettlement growth on reproduction rather than pre-settlement growth processes and (ii) I did not know how well intercirculus spacing corresponded with growth for larval fish. Results from the linear regression between total length and circuli number indicate that measurements taken from the sixth circuli capture growth occurring after $\sim 28 \mathrm{~mm}$ in total length.

It should be noted that this preliminary study was conducted to generally inform scale measurement procedures for Chapter 4, the sample size in this pilot study was quite small and I did not undertake rigorous light trap sampling throughout the season or between source populations. Therefore, there is the possibility that for certain fish measured in Chapter 4, part of the measured growth may have occurred immediately before settlement rather than after settlement, or that size at scale formation may have differed between sampling periods due to seasonal variation. In order to dampen the effects of this variation on my estimates of early growth rates, I averaged growth rates over circuli 6-16 to obtain a broad representation of growth that would be less susceptible to minor variation in timing of scale formation and initial scale size. 


\section{Appendix C - Sensitivity Analysis for Deming regression}




\section{Sensitivity analysis}

In order to examine how sensitive the Deming regression analysis was to the selected variance ratio (the mean variance for both survival time and larval length) I re-ran the regression 1000 times using a randomly selected variance ratio. The variance ratio was calculated by randomly selecting a variance value from the possible variance range for both average larval length (harbour $\sigma^{2}$ range $=0.0105-0.0697$ ), south coast $\sigma^{2}$ range $=0.0111-0.0599$ ) and average survival time (harbour $\sigma^{2}$ range $=1.46-4.00$, south coast $\sigma^{2}$ range $=1.153-4.389$ ). I then plotted each of the regression estimates obtained from the simulations against variation in both length and survival (harbour - Fig C1, south coast- Fig. C2). In addition, I then calculated how many of those regression estimates had confidence intervals that overlapped with zero (i.e., were not significantly different than zero). For the harbour regression, all of the regression estimates overlapped with 0 . For the south coast, only $1.2 \%(\mathrm{n}=12)$ of all regression estimates had confidence intervals that overlapped with 0 , suggesting that the significance of the results for the south coast regression were not particularly sensitive to the selected variance ratio. 

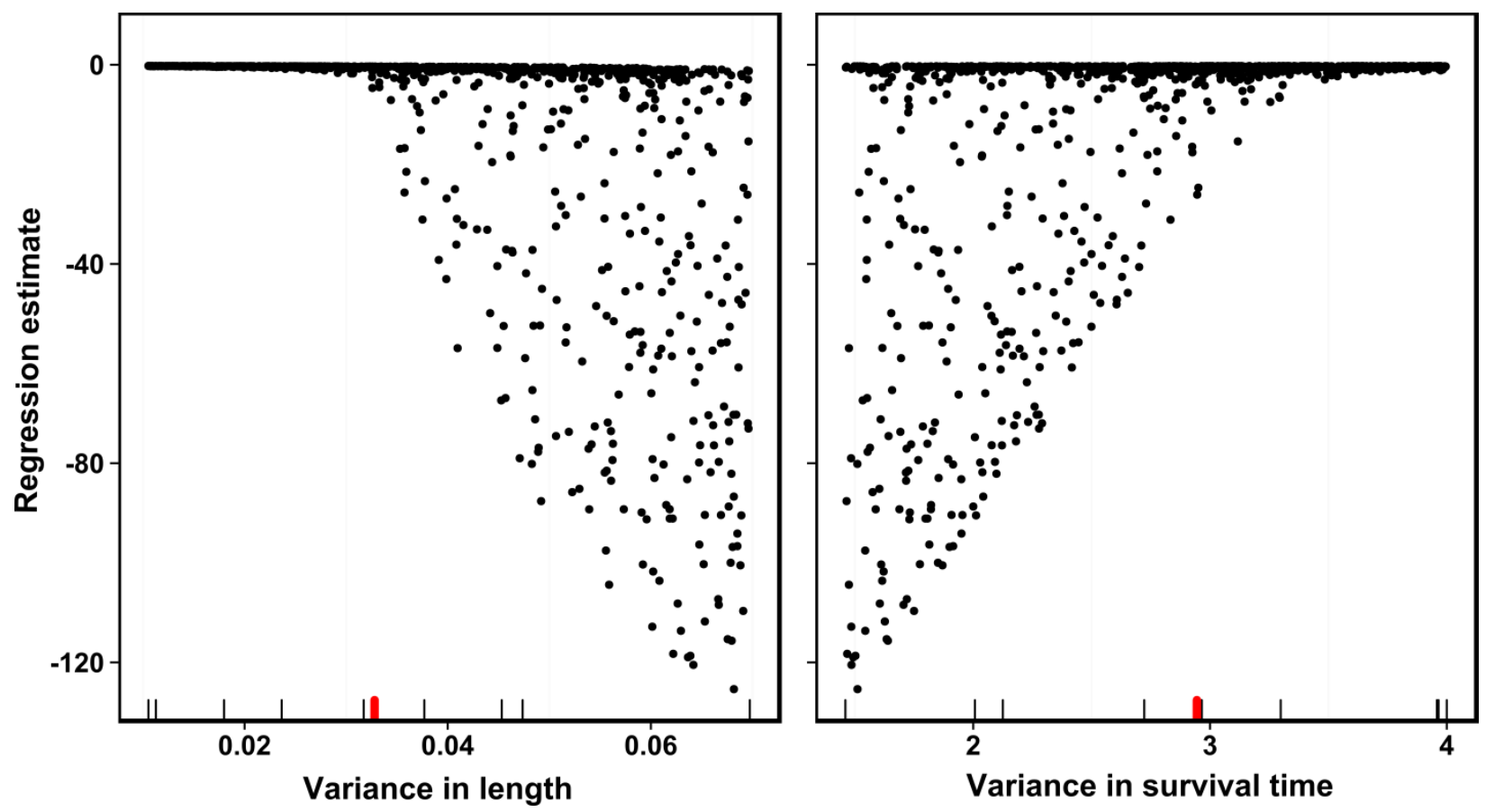

Figure C1 1000 regression estimates for the relationship between mean survival time and mean larval size from the harbour using randomly selected variance ratios for both mean survival time and mean length. Left panel represents how the regression estimates changed with variance in average length and right panel shows how the regression estimates change with variance in survival time (regression estimates for each panel are both from the same pool of 1000). Black rug marks across the bottom axis represent the actual variance measurements from each nest and the red rug mark indicates the mean variance value, which was used in the selected model. 

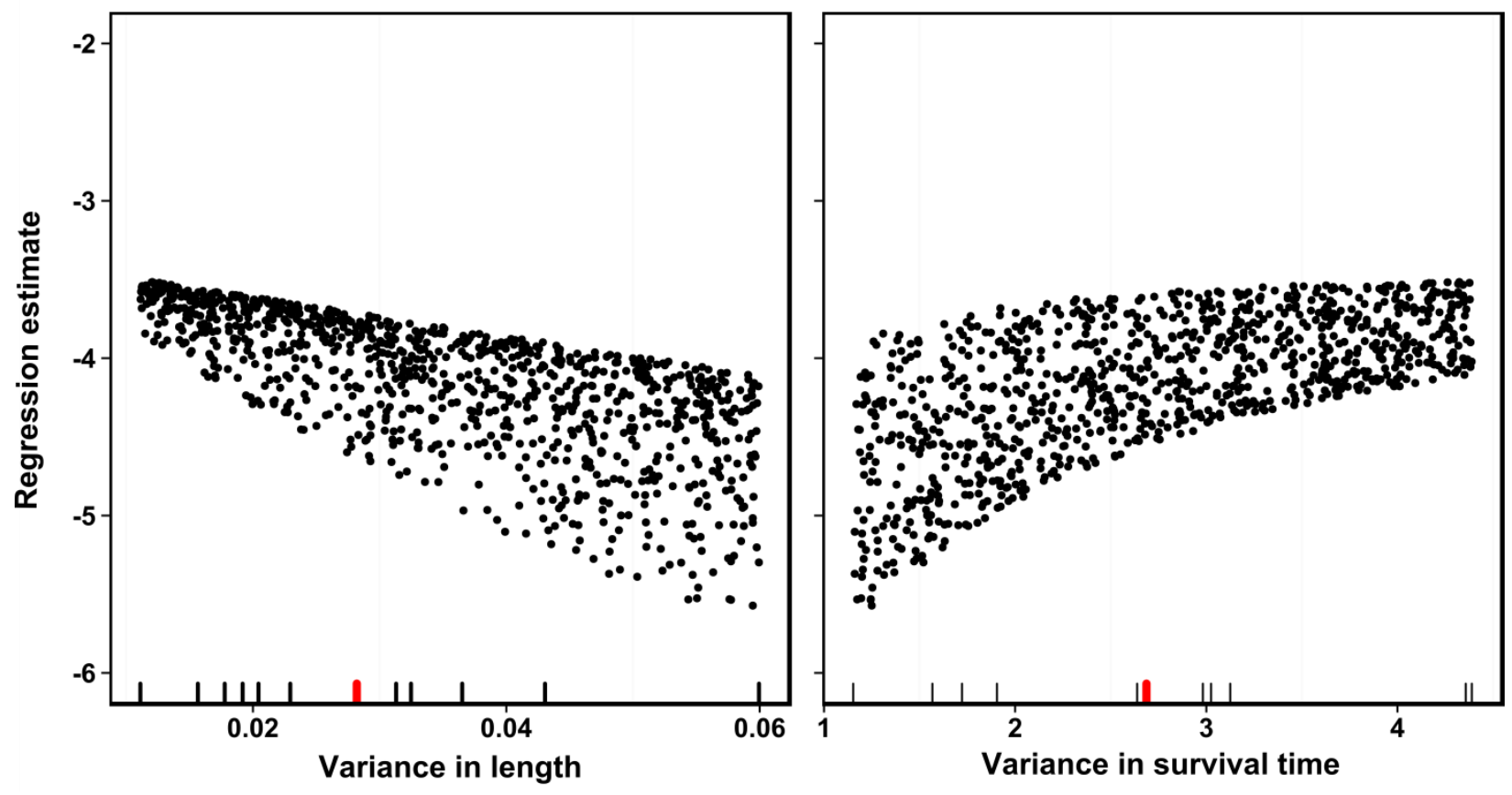

Figure C2 1000 regression estimates for the relationship between mean survival time and mean larval size from the south coast using randomly selected variance ratios for both mean survival time and mean length. Left panel represents how the regression estimates changed with variance in average length and right panel shows how the regression estimates change with variance in survival time (regression estimates for each panel are both from the same pool of 1000). Black rug marks across the bottom axis represent the actual variance measurements from each nest and the red rug mark indicates the mean variance value, which was used in the selected model. 


\section{Appendix D - Simple simulation for larval mortality from two source populations}




\section{Simulation}

To estimate the potential for differences in the relationship between larval size and starvation mortality between natal sources to affect population connectivity, I ran a simplified simulation based on the data in Chapter 6 (Fig. D1):

Step 1: I created 10 nests at each source population and then assigned each nest an average larval size using the mean and standard deviation of average larval sizes for each source population.

Step 2: For each nest, I calculated an average survival estimate (i.e., average number of days survived for larvae in the simulated nest) using its assigned average larval size (calculated in step 1) along with the Deming regression equation (intercept and slope).

Step 3: Finally, I randomly generated 1000 "larvae" from each nest and assigned it a survival time (number of days until death) using the mean survival time (calculated in step 2) and the average standard deviation of survival time for nests within each location assuming a normal distribution.

Step 4: I ran this simulation 100 times and I then plotted the mean number of larvae surviving on each day post-hatch.

I assumed equal reproductive output from each natal source (10000 larvae) and homogenous mixing in the larval pool. In addition, I did not take into account error in calculating the regression slope for the Deming regression (i.e., I only used the regression estimates themselves and no attempt was made to integrate the error associated in calculating those regression estimates into the simulation). It is important to note that this simulation is meant to illustrate the relative contribution of source populations to the larval pool that may occur under certain circumstances, and is not intended to be a predictive model. 

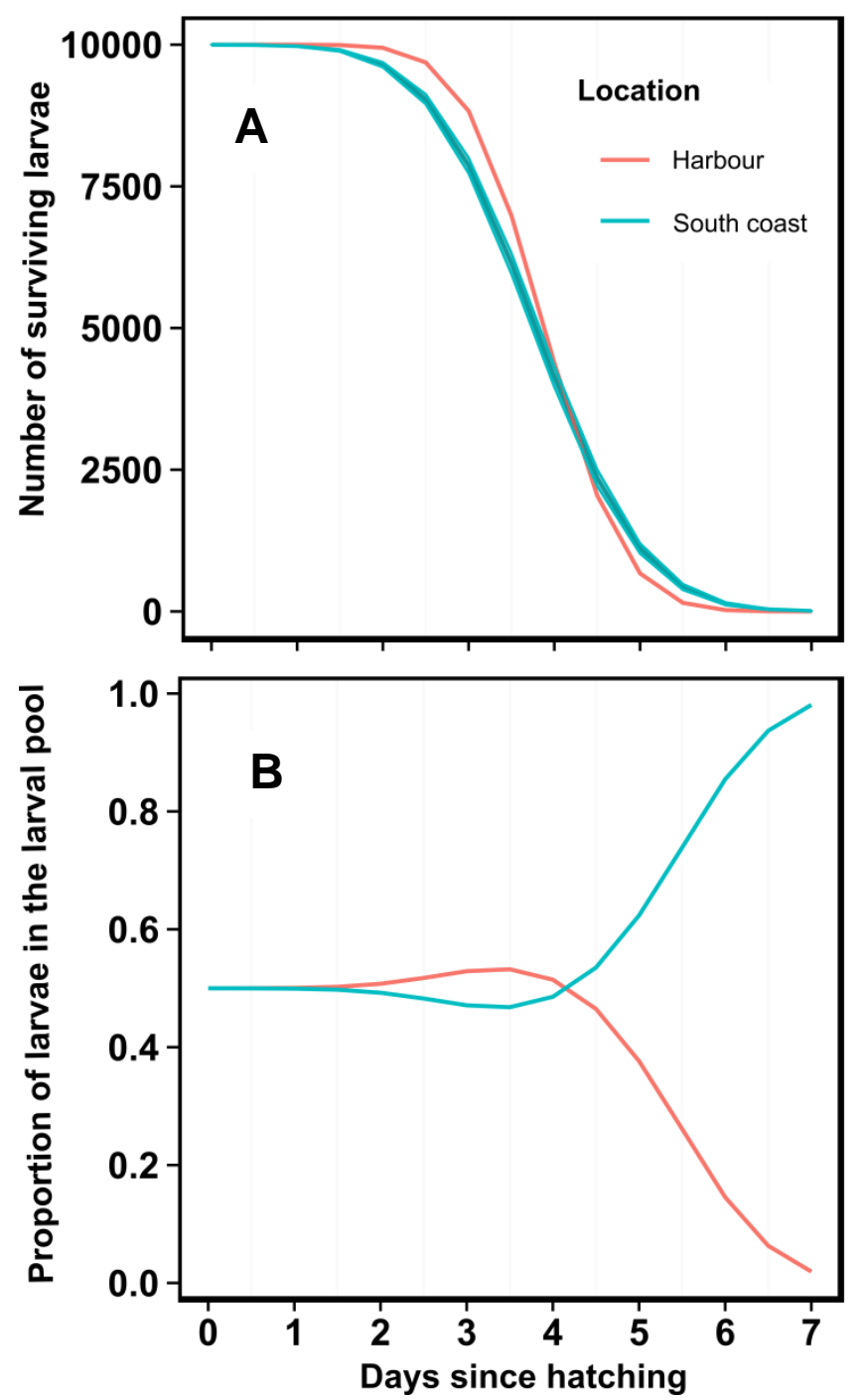

Figure D1A Simulation of the number of surviving larvae per day since hatching and $\mathbf{B}$ Percentage of larvae in the larval pool from each source population. Confidence intervals are plotted as shaded bands around the mean line; however, confidence intervals were extremely small and are only visible for the south coast as a slight thickening of the line (panel A). If food becomes available early on in larval development (Days 1 and 2) then the relative contribution of each natal source to the overall larval pool is very similar. If food becomes available on days 3 and 4 , the harbour contributes slightly more larvae to the pool because the larger larvae on the south coast have begun to die off earlier. If food does not become available until later on days 5 and 6 , then the south coast provides the majority of the larvae to the larval pool. This simulation is intended for illustrative purposes only and is not considered an actual predictive model. 


\section{Literature Cited}

Able D (1996) The contagion indicator hypothesis for parasite-mediated sexual selection. Proc Natl Acad Sci USA 93:2229-2233. doi: 10.1073/pnas.93.5.2229

Adam TC (2011) High-quality habitat and facilitation ameliorate competitive effects of prior residents on new settlers. Oecologia 166:121-130. doi: 10.1007/s00442-010-1826-7

Adlard RD, Lester RJ (1994) Dynamics of the interaction between the parasitic isopod, Anilocra pomacentri, and the coral reef fish, Chromis nitida. Parasitology 109:311-24.

Akaike H (1974) A new look at the statistical model identification. Autom Control IEEE Trans 19:716-723.

Almany GR (2004) Differential effects of habitat complexity, predators and competitors on abundance of juvenile and adult coral reef fishes. Oecologia 141:105-113.

Almany GR, Berumen ML, Thorrold SR, et al. (2007) Local replenishment of coral reef fish populations in a marine reserve. Science $316: 742-744$.

Almany GR, Connolly SR, Heath DD, et al. (2009) Connectivity, biodiversity conservation and the design of marine reserve networks for coral reefs. Coral Reefs 28:339-351. doi: $10.1007 / \mathrm{s} 00338-009-0484-\mathrm{X}$

Almany GR, Webster MS (2006) The predation gauntlet: early post-settlement mortality in reef fishes. Coral Reefs 25:19-22. doi: 10.1007/s00338-005-0044-y

Alvord W (1954) Validity of age determinations from scales of brown trout, rainbow trout, and brook trout. Trans Am Fish Soc 83:91-103. doi: 10.1577/15488659(1953)83[91:VOADFS]2.0.CO;2

Amara R, Meziane T, Gilliers C, et al. (2007) Growth and condition indices in juvenile sole Solea solea measured to assess the quality of essential fish habitat. Mar Ecol Prog Ser 351:201-208. doi: 10.3354/meps07154

Anderson MJ, Millar RB (2004) Spatial variation and effects of habitat on temperate reef fish assemblages in northeastern New Zealand. J Exp Mar Bio Ecol 305:191-221. doi: http://dx.doi.org/10.1016/j.jembe.2003.12.011

Anderson T (2001) Predator responses, prey refuges, and density-dependent mortality of a marine fish. Ecology 82:245-257.

Arendt J (1997) Adaptive intrinsic growth rates: an integration across taxa. Q Rev Biol 72:149177. 
Ayllón D, Nicola GG, Parra I, et al. (2013) Intercohort density dependence drives brown trout habitat selection. Acta Oecologica 46:1-9. doi: 10.1016/j.actao.2012.10.007

Bailey KM, Houde ED (1989) Predation on eggs and larvae of marine fishes and the recruitment problem. Adv. Mar Biol. 25:1-83. http://dx.doi.org/10.1016/S0065-2881(08)60187-X

Barber I (2002) Parasites, male-male competition and female mate choice in the sand goby. J Fish Biol 61:185-198. doi: 10.1006/jfbi.2002.2031

Barber I (2013) Sticklebacks as model hosts in ecological and evolutionary parasitology. Trends Parasitol 29:556-66. doi: 10.1016/j.pt.2013.09.004

Barber I, Hoare D, Krause J (2000) Effects of parasites on fish behaviour: a review and evolutionary perspective. Rev Fish Biol Fish 131-165.

Barber I, Arnott SA, Braithwaite VA, et al. (2001) Indirect fitness consequences of mate choice in sticklebacks: offspring of brighter males grow slowly but resist parasitic infections. Proc Biol Sci 268:71-6. doi: 10.1098/rspb.2000.1331

Barton L (2013) MuMIn: Multi-model inference. R package version 1.9.5. http://CRAN.Rproject.org/package $=$ MuMIn

Bates D, Maechler M, Bolker B, Walker S (2013) lme4: Linear mixed-effects models using Eigen and S4. R package version 1.0-4. http://CRAN.R-project.org/package=lme4

Bay LK, Crozier RH, Caley MJ (2006) The relationship between population genetic structure and pelagic larval duration in coral reef fishes on the Great Barrier Reef. Mar Biol 149:1247-1256. doi: 10.1007/s00227-006-0276-6

Beakes MP, Sharron S, Charish R, et al. (2014) Using scale characteristics and water temperature to reconstruct growth rates of juvenile steelhead Oncorhynchus mykiss. J Fish Biol 84:58-72. doi: 10.1111/jfb. 12254

Beckers OM, Wagner WE (2013) Parasitoid infestation changes female mating preferences. Anim Behav 85:791-796. doi: 10.1016/j.anbehav.2013.01.025

Bellwood DR, Hughes TP, Folke C, Nystrom M (2004) Confronting the coral reef crisis. Nature 429:827-833.

Bendix C, Lyle G, Ekstrom C, Figurski M (2013) MethComp: Functions for analysis of agreement in method comparison studies. R package version 1.22. http://CRAN.Rproject.org/package $=$ MethComp 
Ben-Tzvi O, Kiflawi M, Polak O, Abelson A (2009) The effect of adult aggression on habitat selection by settlers of two coral-dwelling damselfishes. PLoS One 4:8. doi: e5511 10.1371/journal.pone.0005511

Berkeley S, Chapman C, Sogard S (2004) Maternal age as a determinant of larval growth and survival in a marine fish, Sebastes melanops. Ecology 85:1258-1264.

Beukers JS, Jones GP (1998) Habitat complexity modifies the impact of piscivores on a coral reef fish population. Oecologia 114:50-59. doi: 10.1007/s004420050419

Binning SA, Roche DG, Layton C (2013) Ectoparasites increase swimming costs in a coral reef fish. Biol Lett 9 (1). doi: 10.1098/rsbl.2012.0927

Birkeland C, Dayton PK (2005) The importance in fishery management of leaving the big ones. Trends Ecol Evol 20:356-358.

Bjørnstad O, Fromentin J, Stenseth N, Gjøsaeter J (1999) Cycles and trends in cod populations. Proc Natl Acad Sci USA 96:5066-71. doi: 10.1073/pnas.96.9.5066

Bjørnstad O, Nisbet RM, Fromentin JM (2004) Trends and cohort resonant effects in age structured populations. J Anim Ecol 73:1157-1167. doi: 10.1111/j.0021-

8790.2004.00888.x

Bochdansky AB, Grønkjær P, Herra TP, Leggett WC (2005) Experimental evidence for selection against fish larvae with high metabolic rates in a food limited environment. Mar Biol 147:1413-1417. doi: 10.1007/s00227-005-0036-z

Bolker BM, Brooks ME, Clark CJ, et al. (2009) Generalized linear mixed models: a practical guide for ecology and evolution. Trends Ecol Evol 24:127-35. doi:

10.1016/j.tree.2008.10.008

Booman C, Folkvord A, Hunter JR (1991) Responsiveness of starved northern anchovy engraulis-mordax larvae to predatory attacks by adult anchovy. Fish Bull 89:707-711.

Booth DJ, Brosnan DM (1995) The role of recruitment dynamics in rocky shore and coral reef fish communities. Advances in Ecological Research 26: 309-385.

Booth JD (1975) Seasonal and tidal variations in the hydrology of Wellington harbour. New Zeal J Mar Freshw Res 9:333-354. doi: 10.1080/00288330.1975.9515572

Borg AA, Forsgren E, Amundsen T (2006) Seasonal change in female choice for male size in the two-spotted goby. Anim Behav 72:763-771. doi: 10.1016/j.anbehav.2005.11.025

Borgia G (1986) Satin bowerbird parasites: a test of the bright male hypothesis. Behav Ecol Sociobiol 19: 355-358. 
Borgstem R, Heggenes J, Nothcote TG (1993) Regular, cyclic oscillations in cohort strength in an allopatric population of brown trout. Salmo trutta L. Ecol Freshw Fish 2:8-15. doi: 10.1111/j.1600-0633.1993.tb00011.x

Bowman M, Kibblewhite A, Murtagh RA, et al. (1983) Circulation and mixing in greater Cook Strait, New Zealand. Oceanol Acta 6:383-391.

Boxshall GA (1974) Infections with Parasitic Copepods in North Sea Marine Fishes. J Mar Biol Assoc United Kingdom 54:355-372. doi: 10.1017/S0025315400058598

Bravo S (2010) The reproductive output of sea lice Caligus rogercresseyi under controlled conditions. Exp Parasitol 125:51-4. doi: 10.1016/j.exppara.2009.12.001

Britton JR (2010) Scale circuli patterns differentiate between hatchery-reared and wild Rutilus rutilus during evaluation of fish stocking. J Fish Biol 77:2454-9. doi: 10.1111/j.10958649.2010.02800.x

Brooks, S, Tyler, CR, Sumpter, JP. 1997. Egg quality in fish: what makes a good egg? Reviews in Fish Biology and Fisheries. 7:387-416

Brothers E, Williams D, Sale P (1983) Length of larval life in twelve families of fishes at "One Tree Lagoon", Great Barrier Reef, Australia. Mar Biol 324:319-324.

Burnham KP, Anderson DR (2002) Model selection and multimodel inference: a practical information-theoretic approach. Ecol Modell 172:488. doi: 10.1016/j.ecolmodel.2003.11.004

Cable J, van Oosterhout C (2007) The impact of parasites on the life history evolution of guppies (Poecilia reticulata): the effects of host size on parasite virulence. Int J Parasitol 37:1449-58. doi: 10.1016/j.ijpara.2007.04.013

Caley M, Carr M, Hixon M, et al. (1996) Recruitment and the local dynamics of open marine populations. Annu Rev Ecol Syst 27:477-500. doi: 10.1146/annurev.ecolsys.27.1.477

Campana S (2001) Accuracy, precision and quality control in age determination, including a review of the use and abuse of age validation methods. J Fish Biol 59:197-242. doi: 10.1006/jfbi.2001.1668

Campana SE (1990) How reliable are growth back-caluclations based on otoliths? Can J Fish Aquat Res 47:2219-2227.

Campana SE (2005) Otolith science entering the 21st century. Mar Freshw Res 56:485-495.

Campana SE, Thorrold SR (2001) Otoliths, increments and elements: keys to a comprehensive understanding of fish populations? Can J Fish Aquat Sci 58:30-38. 
Canty A, Ripley B (2013) Boot: Bootstrap R (S-Plus) Functions. R package version 1.3-9.

Cargnelli LM, Gross MR (1996) The temporal dimension in fish recruitment: birth date, body size, and size-dependent survival in a sunfish (bluegill : Lepomis macrochirus). 367:360367.

Carr MH (1994) Effects of macroalgal dynamics on recruitment of a temperate reef fish. Ecology 75:1320. doi: $10.2307 / 1937457$

Castro L, Claramunt G, Krautz M, et al. (2009) Egg trait variation in anchoveta Engraulis ringens: a maternal response to changing environmental conditions in contrasting spawning habitats. Mar Ecol Prog Ser 381:237-248. doi: 10.3354/meps07922

Cermeno P, Uriarte A, De Murguia A, Morales-Nin (2003) Validation of daily increment formation in otoliths of juvenile and adult European anchovy. J Fish 62:679-691. doi: 10.1046/j.0022-1112.2003.00056.x

Chambers R, Leggett W (1996) Maternal influences on variation in egg sizes in temperate marine fishes. Am Zool 36:180-196.

Chapman M, Kramer D (2000) Movements of fishes within and among fringing coral reefs in Barbados. Environ Biol Fishes 57:11-24.

Cheney KL, Côté IM (2003) The ultimate effect of being cleaned: does ectoparasite removal have reproductive consequences for damselfish clients? Behav Ecol 14:892-896. doi: 10.1093/beheco/arg079

Cheung CHY, Chaillé PM, Randall DJ, et al. (2007) The use of scale increment as a means of indicating fish growth and growth impairment. Aquaculture 266:102-111. doi:

10.1016/j.aquaculture.2007.02.036

Choat J (1982) Fish feeding and the structure of benthic communities in temperate waters. Annu Rev Ecol Syst 13:423-449.

Clayton D (1991) The influence of parasites on host sexual selection. Parasitol Today 7:329-334. doi: 10.1016/0169-4758(91)90211-6

Clayton DH, Pruett-Jones SG, Lande R (1992) Reappraisal of the interspecific prediction of parasite-mediated sexual selection: Opportunity knocks. J Theor Biol 157:95-108. doi: $10.1016 /$ S0022-5193(05)80759-3

Clements KD (2003) Triplefins. In: Andrew N, Francis M (eds) The Living Reef: The Ecology of New Zealand's Rocky Reefs. Craig Potton Publishing, Nelson, pp 160-167 
Clutton-Brock, Tim H (1988) Reproductive Success: Studies of Individual Variation in Contrasting Breeding Systems. University of Chicago Press

Cobcroft JM, Battaglene SC (2009) Jaw malformation in striped trumpeter Latris lineata larvae linked to walling behaviour and tank colour. Aquaculture 289:274-282. doi:

10.1016/j.aquaculture.2008.12.018

Connell S, Jones G (1991) The influence of habitat complexity on postrecruitment processes in a temperate reef fish population. J Exp Mar Bio Ecol 151:271-294. doi:

http://dx.doi.org/10.1016/0022-0981(91)90129-K

Costello MJ (2006) Ecology of sea lice parasitic on farmed and wild fish. Trends Parasitol 22:475-83. doi: 10.1016/j.pt.2006.08.006

Costello MJ (2009) How sea lice from salmon farms may cause wild salmonid declines in Europe and North America and be a threat to fishes elsewhere. Proc Biol Sci 276:3385-94. doi: $10.1098 /$ rspb.2009.0771

Côté I, Hunte W (1989) Male and female mate choice in the Redlip blenny: why bigger is better. Anim Behav 38:78-88. doi: http://dx.doi.org/10.1016/S0003-3472(89)80067-3

Cowen RK, Lwiza KMM, Sponaugle S, et al. (2000) Connectivity of marine populations: open or closed? Science 287:857-859.

Cowen RK, Paris CB, Srinivasan A (2006) Scaling of connectivity in marine populations. Science 311:522-7. doi: 10.1126/science.1122039

Cowen RK, Sponaugle S (2009) Larval dispersal and marine population connectivity. Ann Rev Mar Sci 1:443-466. doi: 10.1146/annurev.marine.010908.163757

Crispin K (1998) Fish Larvae of the Wellington Harbour and South Coast. MSc Thesis. Victoria University of Wellington. $165 \mathrm{pp}$.

Cushing DH (1975) Marine ecology and fisheries. Cambridge Univ. Press, Cambridge, 278 p

Doherty PJ, Dufour V, Galzin R, Hixon MA, Planes S (2004) High mortality during settlement is a population bottleneck for a tropical surgeonfish. Ecology 85:2422-2428.

Donelson JM, McCormick MI, Munday PL (2008) Parental condition affects early life-history of a coral reef fish. J Exp Mar Bio Ecol 360:109-116. doi: 10.1016/j.jembe.2008.04.007

Downhower JF, Brown L (1980) Mate preferences of female mottled sculpins, Cottus bairdi. Anim Behav 28:728-734. doi: 10.1016/S0003-3472(80)80132-1 
Doyle RW, Talbot AJ, Nicholas RR (1987) Statistical interrelation of length, growth, and scale circulus spacing: appraisal of a growth rate estimator for fish. Can J Fish Aquat Sci 44:1520-1528.

Eckman JE (1996) Closing the larval loop: linking larval ecology to the population dynamics of marine benthic invertebrates. J Exp Mar Bio Ecol 200:207-237. doi: 10.1016/S00220981(96)02644-5

Efron, B (1987) Better bootstrap confidence intervals. Journal of American statistical Association 82:171-185.

Ehman KD, Scott ME (2002) Female mice mate preferentially with non-parasitized males. Parasitology 125:461-6. doi: 10.1017/S003118200200224X

Emlen S, Oring L (1977) Ecology, sexual selection, and the evolution of mating systems. Science 197:215-223.

Endler J, Lyles A (1989) Bright ideas about parasites. Trends Ecol. Evol. 4:246-248

Feary DA, Clements KD (2006) Habitat use by triplefin species (Tripterygiidae) on rocky reefs in New Zealand. J Fish Biol 69:1031-1046. doi: 10.1111/j.1095-8649.2006.01178.x

Feary DA, Wellenreuther M, Clements KD (2009) Trophic ecology of New Zealand triplefin fishes (Family Tripterygiidae). Mar Biol 156:1703-1714. doi: 10.1007/s00227-009-12052

Finley R, Forrester G (2003) Impact of ectoparasites on the demography of a small reef fish. Mar Ecol Prog Ser 248:305-309. doi: 10.3354/meps248305

Fisher JP, Pearcy WG (1990) Spacing of scale circuli versus growth rate in young coho salmon. Fish. Bull. 4:637-643.

Fisher J, Pearcy W (2005) Seasonal changes in growth of coho salmon (Oncorhynchus kisutch) off Oregon and Washington and concurrent changes in the spacing of scale circuli. Fish Bull 103:34-51.

Forrester GE (1995) Strong density-dependent survival and recruitment regulate the abundance of a coral reef fish. Oecologia 103:275-282. doi: 10.1007/BF00328615

Forrester GE (1999) The influence of adult density an larval settlement in a coral reef fish, Coryphopterus glaucofraenum. Coral Reefs 18:85-89. doi: 10.1007/s003380050159

Forrester GE, Evans B, Steele MA, Vance RR (2006) Assessing the magnitude of intra-and interspecific competition in two coral reef fishes. Oecologia 148:632-640. doi:

$10.1007 / \mathrm{s} 00442-006-0397-0$ 
Fortier L, Leggett WC (1985) A drift study of larval fish survival. Mar Ecol Ser 25:245-257. doi: $10.3354 /$ meps025245

Foster S, Garcia V, Town M (1988) Cannibalism as the cause of an ontogenetic shift in habitat use by fry of the threespine stickleback. Oecologia 74:577-585. doi:

10.1007/BF00380056

Fouda MM (1979) Studies on scale regeneration in the Common goby, Pomatoschistus microps (Pisces). J Zool 189:503-522.

Francis M, (2012) Coastal Fishes of New Zealand. Craig Potton Publishing, Nelson. pp 267

Francis RICC (1990) Back-calculation of fish length: a critical review. J Fish Biol 36:883-902. doi: 10.1111/j.1095-8649.1990.tb05636.x

Frederick J (1997) Post-settlement movement of coral reef fishes and bias in survival estimates. Mar Ecol Prog Ser 150:65-74

Friedland K (2000) Linkage between ocean climate, post-smolt growth, and survival of Atlantic salmon (Salmo salar L.) in the North Sea area. ICES J Mar Sci 57:419-429. doi: 10.1006/jmsc. 1999.0639

Fukuwaka M, Kaeriyama M (1997) Scale analyses to estimate somatic growth in sockeye salmon, Oncorhynchus nerka. Can J Fish Aquat Sci 54:631-636.

Gagliano M, McCormick MI, Meekan MG (2007) Survival against the odds: ontogenetic changes in selective pressure mediate growth-mortality trade-offs in a marine fish. Proc Biol Sci 274:1575-82. doi: 10.1098/rspb.2007.0242

Geange SW, Stier AC (2009) Order of arrival affects competition in two reef fishes. Ecology 90:2868-2878. doi: 10.1890/08-0630.1

Gelman A, Hill J (2007) Data Analysis Using Regression and Multileval/Hierarchical Models. Cambridge University Press, Cambridge.

Gimenez L (2006) Phenotypic links in complex life cycles: conclusions from studies with decapod crustaceans. Integr Comp Biol 46:615-622. doi: 10.1093/icb/ic1010

Gimenez L, Anger K, Torres G (2004) Linking life history traits in successive phases of a complex life cycle: effects of larval biomass on early juvenile development in an estuarine crab, Chasmagnathus granulata. Oikos 104:570-580.

González L, Carvajal J (2003) Life cycle of Caligus rogercresseyi, (Copepoda: Caligidae) parasite of Chilean reared salmonids. Aquaculture 220:101-117. doi: 10.1016/S00448486(02)00512-4 
Gotthard K, Nylin S, Wiklund C (1994) Adaptive variation in growth rate: life history costs and consequences in the speckled wood butterfly, Pararge aegeria. Oecologia 99:281-289

Griffen BD (2006) Detecting emergent effects of multiple predator species. Oecologia 148:702709. doi: 10.1007/s00442-006-0414-3

Grueber CE, Nakagawa S, Laws RJ, Jamieson IG (2011) Multimodel inference in ecology and evolution: challenges and solutions. J Evol Biol 24:699-711. doi: 10.1111/j.14209101.2010.02210.x

Grutter A (1994) Spatial and temporal variations of the ectoparasites of seven reef fish species from Lizard Island and Heron Island, Australia. Mar Ecol Prog Ser 115:21-30. doi: 10.3354/meps 115021

Gutiérrez L (1998) Habitat selection by recruits establishes local patterns of adult distribution in two species of damselfishes: Stegastes dorsopunicans and S. planifrons. Oecologia 115:268-277. doi: 10.1007/s004420050516

Hakkarainen H, Ilmonen P, Koivunen V, Korpimäki E (1998) Blood parasites and nest defense behaviour of Tengmalm's owls. Oecologia 114: 574-577. doi: 10.1007/s004420050482

Hamilton SL, Regetz J, Warner RR (2008) Postsettlement survival linked to larval life in a marine fish. Proc Natl Acad Sci U S A 105:1561-1566.

Hamilton WD (1982) Pathogens as Causes of Genetic Diversity in their Host Populations. In: Anderson RM, May RM (eds) Popul. Biol. Infect. Dis. Springer Berlin Heidelberg, Berlin, Heidelberg, pp 269-296

Hamilton WD, Zuk M (1982) Heritable true fitness and bright birds: a role for parasites? Science 218: 384-387. doi: 10.1126/science. 7123238

Hamilton W, Poulin R (1997) The Hamilton and Zuk hypothesis revisited: a meta-analytical approach. Behaviour 134:299-320.

Handford C (1979) The habitat, population dynamics and social organisation of two tripterygiid fishes. MSc Thesis. University of Auckland.

Hartigan J, Hartigan P (1985) The dip test of unimodality. Ann Stat 13:70-84.

Hastings PA (1988) Female choice and male reproductive success in the angel blenny, Coralliozetus-angelica (teleostei, chaenopsidae). Anim Behav 36:115-124. doi: 10.1016/s0003-3472(88)80254-9

Hastings PA (1992) Nest-site size as a short-term constraint on the reproductive success of paternal fishes. Environ Biol Fishes 34:213-218. doi: 10.1007/BF00002397 
Hastings, P. A (2009). Biogeography of new world blennies In The Biology of Blennies, RA Patzner, EJ Gonçlaves, PA Hastings y BG Kapoor (eds.). Science Publishers, Enfield, New Hampshire pp 95-118.

Hastings, P. A. and C. W. Peterson. 2010. Parental care, oviposition sites, and mating systems of blennioid fishes.in K. Cole, editor. Reproduction and sexuality in marine fishes: patterns and processes. University of California Press, Berkeley. pp91-119

Hayes J, Weikel J, Huso M (2003) Response of birds to thinning young Douglas-fir forests. Ecol Appl 13:1222-1232. doi: http://dx.doi.org/10.1890/02-5068

Heath R (1985) A review of the physical oceanography of the seas around New Zealand - 1982. New Zeal J Mar Freshw Res 19:79-124. doi: 10.1080/00288330.1985.9516077

Hedgecock D (1994) Does variance in reproductive success limit effective population sizes of marine organisms. In: Beaumont A (ed) Genet. Evol. Aquat. Org. Chapman \& Hall, London, pp 122-134

Helson JG, Pledger S, Gardner JPA (2007) Does differential particulate food supply explain the presence of mussels in Wellington Harbour (New Zealand) and their absence on neighbouring Cook Strait shores? Estuar Coast Shelf Sci 72:223-234. doi:

10.1016/j.ecss.2006.10.015।

Herbst SJ, Marsden JE (2011) Comparison of precision and bias of scale, fin ray, and otolith age estimates for lake whitefish (Coregonus clupeaformis) in Lake Champlain. J Great Lakes Res 37:386-389. doi: 10.1016/j.jglr.2011.02.001

Heuschele J, Candolin U (2010) Reversed parasite-mediated selection in sticklebacks from eutrophied habitats. Behav Ecol Sociobiol 64:1229-1237. doi: 10.1007/s00265-010-0937-9

Heuschele J, Mannerla M, Gienapp P, Candolin U (2009) Environment-dependent use of mate choice cues in sticklebacks. Behav Ecol 20:1223-1227. doi: 10.1093/beheco/arp123

Hewitt G (1964) Some Copepoda Parasitic on Fishes of the New Zealand Region. PhD Thesis. Victoria University of Wellington.

Hillgarth N (1996) Ectoparasite transfer during mating in ring-necked pheasants Phasianus colchicus. J Avian Biol 27:260-262. doi: 10.2307/3677232

Hine PM, Jones JB. Diggles BK (2000) A checklist of the parasites of New Zealand fishes, including previously unpublished records. NIWA Technical Report, 75, 1-94

Hirschberg JG, Lye JN (2005) Inferences for the extremum of quadratic regression models. University of Melbourne, Department of Economics, Research Paper Number 906, Melbourne, Victoria, Australia. 
Hixon M, Anderson T, Buch KL, et al. (2012) Density dependence and population regulation in marine fish: a large-scale, long-term field manipulation. Ecol Monogr 82:467-489. doi: http://dx.doi.org/10.1890/11-1525.1

Hixon M, Jones G (2005) Competition, predation, and density-dependent mortality in demersal marine fishes. Ecology 86:2847-2859. doi: http://dx.doi.org/10.1890/04-1455

Hixon MA (2011) 60 Years of coral reef fish ecology: past, present, future. Bull Mar Sci 87:727-765. doi: 10.5343/bms.2010.1055

Hixon MA, Carr MH (1997) Synergistic predation, density dependence, and population regulation in marine fish. Science 277:946-949.

Hjort J (1914) Fluctuations in the great fishers of northern Europe viewed in the light of biological research. Int Counc Explor Sea 20:1-228.

Hoelzer G (1992) The ecology and evolution of partial-clutch cannibalism by paternal Cortez damselfish. Oikos 65:113-120.

Hogan F, Friedland KD (2010) Retrospective growth analysis of Atlantic salmon Salmo salar and implications for abundance trends. J Fish Biol 76:2502-20. doi: 10.1111/j.10958649.2010.02650.x

Holbrook S, Carr M, Schmitt RJ, Coyer JA (1990) Effect of giant kelp on local abundance of reef fishes: the importance of ontogenetic resource requirements. Bull Mar Sci 47:104114.

Holbrook S, Schmitt R (2002) Competition for shelter space causes density-dependent predation mortality in damselfishes. Ecology 83:2855-2868.

Hothorn T, Bretz F, Westfall P (2008) Simultaneous inference in general parametric models. Biometrical Journal 50:346-363

Houde AE, Torio AJ (1992) Effect of parasitic infection on male color pattern and female choice in guppies. Behav Ecol 3:346-351. doi: 10.1093/beheco/3.4.346

Houde E (1989) Comparative growth, mortality, and energetics of marine fish larvae: temperature and implied latitudinal effects. Fish. Bull 87:471-495

Howard RD, Micheulla DJ (1990) Parasitism and mate competition. Oikos. 58:120-122

Hoxmeier R, Aday D, Wahl D (2001) Factors influencing precision of age estimation from scales and otoliths of bluegills in Illinois reservoirs. North Am J Fish Manag 21:374-380. doi: $10.1577 / 1548-8675(2001) 021<0374$ 
Hughes T (1994) Catastrophes, phase shifts, and large-scale degradation of a Caribbean coral reef. Science 265:1547-1551.

Hunt T, Ford J, Swearer S (2011) Ecological determinants of recruitment to populations of a temperate reef fish, Trachinops caudimaculatus (Plesiopidae). Mar Freshw Res 62:502509. doi: http://dx.doi.org/10.1071/MF10262

Ibáñez AL, Britton JR, Cowx IG (2008) Relationship between scale growth checks, circuli formation rate and somatic growth in Rutilus rutilus (L.) a fish farm-reared cyprinid. J Fish Biol 72:1023-1034. doi: 10.1111/j.1095-8649.2007.01781.x

Ikoma T, Kobayashi H, Tanaka J, et al. (2003) Microstructure, mechanical, and biomimetic properties of fish scales from Pagrus major. J Struct Biol 142:327-333. doi: 10.1016/S1047-8477(03)00053-4

Islam MS, Ueno M, Yamashita Y (2010) Growth-dependent survival mechanisms during the early life of a temperate seabass (Lateolabrax japonicus): field test of the "growthmortality" hypothesis. Fish Oceanogr 19:230-242. doi: 10.1111/j.1365-2419.2010.00539.x

James MK, Armsworth PR, Mason LB, Bode L (2002) The structure of reef fish metapopulations: modelling larval dispersal and retention patterns. Proc Biol Sci 269:2079-86. doi: 10.1098/rspb.2002.2128

Jawad LA (2005) Comparative scale morphology and squamation patterns in triplefins ( Pisces : Teleostei : Perciformes : Tripterygiidae ). Tuhinga 1879:137-167.

Jennings MJ, Philipp DP (1992) Reproductive investment and somatic growth rates in longear sunfish. Environ Biol Fishes 35:257-271. doi: 10.1007/BF00001892

Jennions MD, Petrie M (1997) Variation in mate choice and mating preferences: A review of causes and consequences. Biol Rev 72:283-327.

Johannes R (1978) Reproductive strategies of coastal marine fishes in the tropics. Environ Biol Fishes 3:65-84.

Johnson DW (2007) Habitat complexity modifies post-settlement mortality and recruitment dynamics of a marine fish. Ecology 88:1716-1725. doi: http://dx.doi.org/10.1890/060591.1

Johnson JB, Omland KS (2004) Model selection in ecology and evolution. Trends Ecol Evol 19:101-8. doi: 10.1016/j.tree.2003.10.013

Johnson SC, Treasurer JW, Bravo S, Nagasawa K (2004) A Review of the Impact of Parasitic Copepods on Marine Aquaculture. ZoolStud 43:229-243. 
Jones GP, (1984) Population ecology of the temperate reef fish Pseudolabrus celidontus Bloch \&Schneider (Pisces: Labridae). I. Factors influencing recruitment. Journal of Experimental Marine Biology and Ecology 75:257-276.

Jones GP (1986) Food availability affects growth in a coral reef fish. Oecologia 70:136-139.

Jones GP (1987) Competitive interactions among adults and juveniles in a coral reef fish. Ecology 68:1534-1547. doi: 10.2307/1939237

Jones G (1990) The importance of recruitment to the dynamics of a coral reef fish population. Ecology 71:1691-1698.

Jones GP, Almany GR, Russ GR, et al. (2009) Larval retention and connectivity among populations of corals and reef fishes: history, advances and challenges. Coral Reefs 28:307-325. doi: 10.1007/s00338-009-0469-9

Jones GP, McCormick MI (2006) Numeric and Energetic Processes in the Ecology of Coral Reef Fishes. In Sale P (ed) Coral Reef Fishes: Dynamics and Diversity in a Complex Ecosystem. Elsevier Publishing, London, pp 221-238

Jones JB (1988) New Zealand parasitic Copepoda; genus Caligus Müller, 1785 (Siphonostomatoida: Caligidae). New Zeal J Zool 15:397-413. doi: 10.1080/03014223.1988.10422966

Kelly CD (2008) The interrelationships between resource-holding potential, resource-value and reproductive success in territorial males: How much variation can we explain? Behav Ecol Sociobiol 62:855-871. doi: 10.1007/s00265-007-0518-8

King HR, Pankhurst NW, Watts M, Pankhurst PM (2003) Effect of elevated summer temperatures on gonadal steroid production, vitellogenesis and egg quality in female Atlantic salmon. J Fish Biol 63:153-167. doi: 10.1046/j.1095-8649.2003.00137.x

Kingsford MJ, Atkinson MH (1994) Increments in otoliths and scales: How they relate to the age and early development of reared and wild larval and juvenile Pagrus auratus (Sparidae). Aust J Mar Freshw Res 45:1007-1021.

Klumb RA, Bozek MA, Frie RV (2001) Validation of three back-calculation models by using multiple oxytetracycline marks formed in the otoliths and scales of bluegill $\times$ green sunfish hybrids. Can J Fish Aquat Sci 58:352-364. doi: 10.1139/cjfas-58-2-352

Knapp R, Kovach J (1991) Courtship as an honest indicator of male parental quality in the bicolor damselfish, Stegastes partitus. Behav Ecol 2: 295-300

Kohn YY, Clements KD (2011) Pelagic larval duration and population connectivity in New Zealand triplefin fishes (Tripterygiidae). Environ Biol Fish 91:275-286. doi: 10.1007/s10641-011-9777-3 
Kokko H, Brooks R, Jennions MD, Morley J (2003) The evolution of mate choice and mating biases. Proc Biol Sci 270:653-64. doi: 10.1098/rspb.2002.2235

Kvarnemo C, Svensson O, Forsgren E (1998) Parental behaviour in relation to food availability in the common goby. Anim Behav 56:1285-1290. doi: 10.1006/anbe.1998.0899

Lecchini D, Planes S, Galzin R (2007) The influence of habitat characteristics and conspecifics on attraction and survival of coral reef fish juveniles. J Exp Mar Bio Ecol 341:85-90. doi: 10.1016/j.jembe.2006.10.006

Lee R (1920) A review of the methods of age and growth determination in fishes by means of scales. Fish Invest 4:32.

Leis J (1991) The pelagic stage of reef fishes: the larval biology of coral reef fishes In Sale PF (eds) The Ecology of fishes on coral reefs. pp 183-230

Levin PS (1991) Effects of microhabitat on recruitment variation in a Gulf of Maine reef fish. Mar Ecol Prog Ser 75:183-189.

Levin PS (1993) Habitat structure, conspecific presence and spatial variation in the recruitment of a temperate reef fish. Oecologia 94:176-185. doi: 10.1007/BF00341315

Lo C, Morand S, Galzin R (1998) Parasite diversity $\backslash$ host age and size relationship in three coralreef fishes from French Polynesia. Int. J. Parasitol. 28:1694-1708

López S (1999) Parasitized female guppies do not prefer showy males. Anim Behav 57:11291134. doi: 10.1006/anbe.1998.1064

Lowe C, Topping D (2003) Movement patterns, home range, and habitat utilization of adult kelp bass Paralabrax clathratus in a temperate no-take marine reserve. Mar Ecol Ser 256:205-216.

Lowry M, Suthers I (1998) Home range, activity and distribution patterns of a temperate rockyreef fish, Cheilodactylus fuscus. Mar Biol 132: 569-578.

Luckhurst BE, Luckhurst K (1978) Analysis of the influence of substrate variables on coral reef fish communities. Mar Biol 49:317-323. doi: 10.1007/BF00455026

Magnhagen C, Kvarnemo L (1989) Big is better: the importance of size for reproductive success in male Pomatoschistus minutus (Pallas) (Pisces, Gobiidae). J Fish Biol 35:755-763. doi: 10.1111/j.1095-8649.1989.tb03027.x

Mangel M, Stamps J (2001) Trade-offs between growth and mortality and the maintenance of individual variation in growth. Evol Ecol 3:583-593. 
Marco-Rius F, Caballero P, Morán P, Garcia de Leaniz C (2013) Mixed-effects modelling of scale growth profiles predicts the occurrence of early and late fish migrants. PLoS One 8:e61744. 8:1-7. doi: 10.1371/journal.pone.0061744

McCallum HI, Kuris A, Harvell CD, et al. (2004) Does terrestrial epidemiology apply to marine systems? Trends Ecol Evol 19:585-591. doi: 10.1016/j.tree.2004.08.009

McDermott CJ, Shima JS (2006) Ontogenetic shifts in microhabitat preference of the temperate reef fish Forsterygion lapillum: implications for population limitation. Mar Ecol Prog Ser 320:259-266. doi: 10.3354/meps320259

Mendiola D, Ibaibarriaga L, Alvarez P (2007) Thermal effects on growth and time to starvation during the yolk-sac larval period of Atlantic mackerel Scomber scombrus L. J Fish Biol 70:895-910. doi: 10.1111/j.1095-8649.2007.01353.x

Meng L, Gray C, Taplin B, Kupcha E (2000) Using winter flounder growth rates to assess habitat quality in Rhode Island's coastal lagoons. Mar Ecol Prog Ser 201:287-299.

Micheli F, Halpern BS (2005) Low functional redundancy in coastal marine assemblages. Ecol Lett 8:391-400. doi: 10.1111/j.1461-0248.2005.00731.x

Milinski M, Bakker T (1990) Female sticklebacks use male coloration in mate choice and hence avoid parasitized males. Nature. 344:330-333 doi: 10.1038/344330a0

Miller TJ, Crowder LB, Rice JA., Marschall EA (1988) Larval Size and Recruitment Mechanisms in Fishes: Toward a Conceptual Framework. Can J Fish Aquat Sci 45:16571670. doi: 10.1139/f88-197

Mitchell MD, McCormick MI, Ferrari MCO, Chivers DP (2011) Coral reef fish rapidly learn to identify multiple unknown predators upon recruitment to the reef. PLoS One 6. doi: e15764 10.1371/journal.pone.0015764

Møller A, Christe P, Lux E (1999) Parasitism, host immune function, and sexual selection. Q Rev Biol 74:3-20. doi: 10.1086/392949

Molony BW, Choat JH (1990) Otolith increment widths and somatic growth rate: the presence of a time-lag. J Fish Biol 37:541-551. doi: 10.1111/j.1095-8649.1990.tb05887.x

Morales-Nin B (1988) Caution in the use of daily increments for ageing tropical fishes. Fishbyte 6:5-6.

Morton DN, Shima JS (2013) Habitat configuration and availability influences the settlement of temperate reef fishes (Tripterygiidae). J Exp Mar Bio Ecol 449:215-220. doi:

10.1016/j.jembe.2013.09.017 
Munday P, Hodges A (2004) Sex-specific growth effects in protogynous hermaphrodites. Can J Fish Aquat Sci 61:323-327. doi: 10.1139/F04-057

Mustafa A, Conboy G, Burka J (2000a) Lifespan and reproductive capacity of sea lice, Lepeophtheirus salmonis, under laboratory conditions. Aquac Assoc Canada, Spec Publ 4:113-114.

Mustafa A, Speare D, Daley J, et al. (2000b) Enhanced susceptibility of seawater cultured rainbow trout, Oncorhynchus mykiss (Walbaum), to the microsporidian Loma salmonae during a primary infection with. J Fish Biol 23:337-341.

Nelson JA, Stallings CD, Landing WM, Chanton J (2013) Biomass transfer subsidizes nitrogen to offshore food webs. Ecosystems 16:1130-1138. doi: 10.1007/s10021-013-9672-1

Neubauer P, Shima J, Swearer S (2010) Scale-dependent variability in Forsterygion lapillum hatchling otolith chemistry: implications and solutions for studies of population connectivity. Mar Ecol Prog Ser 415:263-274. doi: 10.3354/meps08741

Newhard JJ, Love JW, Gill J (2012) Do juvenile white perch Morone americana grow better in freshwater habitats of the blackwater river drainage (Chesapeake Bay, MD, USA)? Estuaries and Coasts 35:1110-1118. doi: 10.1007/s12237-012-9512-0

Oliveira RF, Almada VC, Forsgren E, Goncalves EJ (1999) Temporal variation in male traits, nesting aggregations and mating success in the peacock blenny. J Fish Biol 54:499-512. doi: 10.1111/j.1095-8649.1999.tb00631.x

Oliveira RF, Miranda JA, Carvalho N, et al. (2000) Male mating success in the Azorean rockpool blenny: the effects of body size, male behaviour and nest characteristics. J Fish Biol 57:1416-1428.

Osenberg CW, St. Mary CM, Schmitt RJ, et al. (2002) Rethinking ecological inference: density dependence in reef fishes. Ecol Lett 5:715-721. doi: 10.1046/j.1461-0248.2002.00377.x

Osman RW, Whitlatch RB (2004) The control of the development of a marine benthic community by predation on recruits. J Exp Mar Bio Ecol 311:117-145. doi: 10.1016/j.jembe.2004.05.001

Pedraza-Garcia M, Cubillos LA (2008) Population dynamics of two small pelagic fish in the central-south area off Chile: delayed density-dependence and biological interaction. Environ Biol Fishes 82:111-122. doi: 10.1007/s10641-007-9260-3

Pélabon C, Borg $\AA$, Bjelvenmark J, et al. (2005) Do microsporidian parasites affect courtship in two-spotted gobies? Mar Biol 148:189-196. doi: 10.1007/s00227-005-0056-8 
Pepin P, Orr DC, Anderson JT (1997) Time to hatch and larval size in relation to temperature and egg size in Atlantic cod (Gadus morhua). Can J Fish Aquat Sci 54:2-10. doi:10.1139/f96-154

Pérez-Matus A, Shima JS (2010) Disentangling the effects of macroalgae on the abundance of temperate reef fishes. J Exp Mar Bio Ecol 388:1-10. doi: 10.1016/j.jembe.2010.03.013

Persson L, Byström P, Wahlström E (2000) Cannibalism and competition in Eurasian perch: population dynamics of an ontogenetic omnivore. Ecology 81:1058-1071. doi: . http://dx.doi.org/10.1890/0012-9658(2000)081[1058:CACIEP]2.0.CO;2

Petersen CW (1988) Male mating success, sexual size dimorphism, and site fidelity in two species of Malizcoctenus (Labrisomidae). 21:173-183.

Peyronnet A, Friedland KD, Maoileidigh NÓ, et al. (2007) Links between patterns of marine growth and survival of Atlantic salmon Salmo salar, L. J Fish Biol 71:684-700. doi: 10.1111/j.1095-8649.2007.01538.x

Pierce CL, Rasmussen JB, Leggett WC (1996) Back-calculation of fish length from scales: empirical comparison of proportional methods. Trans Am Fish Soc 125:889-898. doi: 10.1577/1548-8659(1996)125<0889:BCOFLF>2.3.CO;2

Planes S, Jones GP, Thorrold SR (2009) Larval dispersal connects fish populations in a network of marine protected areas. Proc Natl Acad Sci USA 106:5693-7. doi: 10.1073/pnas.0808007106

Platt T, Fuentes-Yaco C, Frank K (2003) Spring algal bloom and larval fish survival. Nature 423:398-399.

Podolsky RD (2003) Integrating development and environment to model reproductive performance in natural populations of an intertidal gastropod. Integr Comp Biol 43:4508. doi: $10.1093 /$ icb/43.3.450

Podolsky RD, Moran AL (2006) Integrating function across marine life cycles. Integr Comp Biol 46:577-586. doi: 10.1093/icb/ic1026

Polis G, Myers C, Holt R (1989) The ecology and evolution of intraguild predation: potential competitors that eat each other. Annu Rev Ecol Syst 20:297-330. doi: http://www.jstor.org/stable/2097094

Possingham H, Roughgarden J (1990) Spatial population dynamics of a marine organism with a complex life cycle. Ecology 71:973-985

Poulin R, Vickery W (1993) Parasite distribution and virulence: implications for parasitemediated sexual selection. Behav Ecol Sociobiol 33:429-436. doi: 10.1007/BF00170258 
Poulin R (1994) Mate choice decisions by parasitized female upland bullies, Gobiomorphus breviceps. Proc R Soc B Biol Sci 256:183-187. doi: 10.1098/rspb.1994.0068

Poulin R, Vickery W (1996) Parasite-mediated sexual selection: just how choosy are parasitized females? Behav Ecol Sociobiol 38:43-49. doi: 10.1007/s002650050215

R Core Team (2013). R: A language and environemtn for statistical computing. R Foundation for Statistical Computing, Vienna, Austria. URL http://www.R=project.org/.

Rana KJ (1985) Influence of egg size on the growth, onset of feeding, point-of-no-return, and survival of unfed Oreochromis mossambicus fry. Aquaculture 46:119-131. doi: $10.1016 / 0044-8486(85) 90196-6$

Raventos N, Macpherson E (2005) Effect of pelagic larval growth and size-at-hatching on postsettlement survivorship in two temperate labrid fish of the genus Symphodus. Mar Ecol Ser 285:205-211.

Reese E (1973) Duration of residence by coral reef fishes on“ home” reefs. Copeia 1973:145149.

Reichard M, Ondracková M, Bryjová A, et al. (2009) Breeding resource distribution affects selection gradients on male phenotypic traits: experimental study on lifetime reproductive success in the bitterling fish (Rhodeus amarus). Evolution 63:377-90. doi: 10.1111/j.1558-5646.2008.00572.x.

Reynolds J, Gross M (1992) Female mate preference enhances offspring growth and reproduction in a fish, Poecilia reticulata. Proc R Soc B Biol Sci 250:57-62.

Richards R, Esteves C (1997) Stock-specific variation in scale morphology of Atlantic striped bass. Trans Am Fish Soc 126:908-918. doi: 10.1577/1548-8659(1997)126<0908

Richards SA (2008) Dealing with overdispersed count data in applied ecology. J Appl Ecol 45:218-227. doi: 10.1111/j.1365-2664.2007.01377.x

Ricker W.E. (1975) Computation and interpretation of biological statistics of fish populations. Bulletin of the Fisheries Research Board of Canada 191, 382 pp

Rohde K, Hayward C, Heap M (1995) Aspects of the ecology of metazoan ectoparasites of marine fishes. Int J Parasitol. 25:945-970 doi: 10.1016/0020-7519(95)00015-T

Rosenqvist G, Johansson K (1995) Male avoidance of parasitized females explained by direct benefits in a pipefish. Anim Behav 49:1039-1045. doi: 10.1006/anbe.1995.0133

Roughgarden J, Gaines S, Possingham H (1988) Recruitment dynamics in complex life cycles. Science 241:1460-1466. doi: DOI: 10.1126/science.11538249 
Sabat AM (1994) Costs and benefits of parental effort in a brood-guarding fish (Ambloplites rupestris, Centrarchidae). Behav Ecol 5:195-201. doi: 10.1093/beheco/5.2.195

Sale P (1971) Extremely limited home range in a coral reef fish, Dascyllus aruanus (Pisces; Pomacentridae). Copeia 2:324-327.

Samhouri JF, Steele MA, Forrester GE (2009) Inter-cohort competition drives density dependence and selective mortality in a marine fish. Ecology 90:1009-1020. doi: $10.1890 / 07-1161.1$

Schielzeth H (2010) Simple means to improve the interpretability of regression coefficients. Methods Ecol Evol 1:103-113. doi: 10.1111/j.2041-210X.2010.00012.x

Schmitt R, Holbrook S, Brooks A, Lape J (2009) Intraguild predation in a structured habitat: distinguishing multiple-predator effects from competitor effects. Ecology 90:2434-2443. doi: http://dx.doi.org/10.1890/08-1225.1

Schmitt RJ, Holbrook SJ (1999) Mortality of juvenile damselfish: implications for assessing processes that determine abundance. Ecology 80:35-50. doi: http://dx.doi.org/10.1890/0012-9658(1999)080[0035:MOJDIF]2.0.CO;2

Semmens D, Swearer S (2012) Trade-offs obscure the relationship between egg size and larval traits in the diadromous fish Galaxias maculatus. Mar Ecol Prog Ser 461:165-174. doi: 10.3354/meps09814

Serbezov D, Bernatchez L, Olsen EM, Vøllestad LA (2010) Mating patterns and determinants of individual reproductive success in brown trout (Salmo trutta) revealed by parentage analysis of an entire stream living population. Mol Ecol 19:3193-205. doi:

10.1111/j.1365-294X.2010.04744.x

Shima JS, McNaughtan D, Geange SW, Wilkinson S (2012) Ontogenetic variation in site fidelity and homing behaviour of a temperate reef fish. J Exp Mar Bio Ecol 416:162-167. doi: 10.1016/j.jembe.2012.02.020

Shima JS, Osenberg CW (2003) Cryptic density dependence: effects of covariation between density and site quality in reef fish. Ecology 84:46-52.

Shima JS, Swearer SE (2009a) Larval quality is shaped by matrix effects: implications for connectivity in a marine metapopulation. Ecology 90:1255-67.

Shima JS, Swearer SE (2009b) Spatially variable larval histories may shape recruitment rates of a temperate reef fish. Mar Ecol Prog Ser 394:223-229. doi: 10.3354/meps08298

Siegel DA, Kinlan BP, Gaylord B, Gaines SD (2003) Lagrangian descriptions of marine larval dispersion. Mar Ecol Prog Ser 260:83-96. 
Sih A, Englund G, Wooster D (1998) Emergent impacts of multiple predators on prey. Trends Ecol Evol 13:350-355. doi: 10.1016/s0169-5347(98)01437-2

Smith A (2010) Environmental and Life-history Factors Influencing Juvenile Demography of a Temperate Reef Fish. PhD Dissertation, Victoria University of Wellington.

Smith AC, Shima JS (2011) Variation in the effects of larval history on juvenile performance of a temperate reef fish. Austral Ecol 36:830-838. doi: 10.1111/j.1442-9993.2010.02223.x

Smith C, Reay P (1991) Cannibalism in teleost fish. Rev Fish Biol Fish 64:41-64.

Sogard SM (1997) Size-selective mortality in the juvenile stage of teleost fishes: A review. Bull Mar Sci 60:1129-1157.

Sogard SM, Olla BL (2002) Contrasts in the capacity and underlying mechanisms for compensatory growth in two pelagic marine fishes. Mar Ecol Prog Ser 243:165-177.

Sparkes TC, Rush V, Kopp DA, Foster SA (2013) Reproductive success in a natural population of male three-spined stickleback Gasterosteus aculeatus: effects of nuptial colour, parasites and body size. J Fish Biol 82:1720-7. doi: 10.1111/jfb.12083

Sponaugle S, Grorud-colvert K (2006) Environmental variability, early life-history traits, and survival of new coral reef fish recruits. Integrative and Comparative Biology,46:623-633

Sponaugle S, Grorud-Colvert K, Pinkard D (2006) Temperature-mediated variation in early life history traits and recruitment success of the coral reef fish Thalassoma bifasciatum in the Florida Keys. Mar Ecol Ser 308:1-15.

Stamps J, Krishnan V (2005) Nonintuitive cue use in habitat selection. Ecology 86:2860-2867. doi: http://dx.doi.org/10.1890/05-0290

Stamps JA (2006) The silver spoon effect and habitat selection by natal dispersers. Ecol Lett 9:1179-1185.

Standish J, Sheehy M, Warner R (2008) Use of otolith natal elemental signatures as natural tags to evaluate connectivity among open-coast fish populations. Mar Ecol Prog Ser 356:259268. doi: $10.3354 /$ meps07244

Stearns SC (2000) Life history evolution: successes, limitations, and prospects. Naturwissenschaften 87:476-86.

Swearer S, Caselle J, Lea D, Warner R (1999) Larval retention and recruitment in an island population of a coral-reef fish. Nature 402:799-802. 
Swearer S, Shima J (2010) Regional variation in larval retention and dispersal drives recruitment patterns in a temperate reef fish. Mar Ecol Prog Ser 417:229-236. doi: $10.3354 /$ meps08801

Sweatman HPA (1988) Field evidence that settling coral reef fish larvae detect resident fishes using dissolved chemical cues. J Exp Mar Bio Ecol 124:163-174. doi: http://dx.doi.org/10.1016/0022-0981(88)90170-0

Sweatman HPA, St John J (1990) Effects of selective settlement and of aggression by residents on distribution of young recruits of 2 tropical damselfishes. Mar Biol 105:247-252. doi: $10.1007 / \mathrm{bf01344293}$

Syms C (1995) Multi-scale analysis of habitat association in a guild of blennioid fishes. Mar Ecol Prog Ser 125:31-43.

Takahashi D (2008) Life-history variation in relation to nest site abundance in males of the freshwater goby Tridentiger brevispinis. Ecol Freshw Fish 17:71-77. doi: 10.1111/j.1600-0633.2007.00260.x

Terry Therneau (2012). coxme: Mixed Effects Cox Models. R package version 2.2-3. http://CRAN.R-project.org/package= coxme

Thompson S (1986) Male spawning success and female choice in the mottled triplefin, Forsterygion varium (Pisces: Tripterygiidae). Anim Behav 34:580-589. doi: http://dx.doi.org/10.1016/S0003-3472(86)80127-0

Thorson G (1950) Reproductive and larval ecology of marine bottom invertebrates. Biol Rev 25:1-45. doi: 10.1111/j.1469-185x.1950.tb00585.x

Thresher, RE.(1984) Reproduction in reef fishes. Neptune City: TFH publications, 1984.

Trivers RL (1972) Parental Investment and Sexual Selection. Aldine Publishing Company, Chicago.

Tobler M, Plath M, Burmeister H, Schlupp I (2006) Black spots and female association preferences in a sexual/asexual mating complex (Poecilia, Poeciliidae, Teleostei). Behav Ecol Sociobiol 60:159-165. doi: 10.1007/s00265-005-0152-2

Tupper M, Boutilier RG (1995) Effects of conspecific density on settlement, growth and postsettlement survival of a temperate reef fish. J Exp Mar Bio Ecol 191:209-222. doi: 10.1016/0022-0981(95)00058-Y

Tupper M, Boutilier RG (1997) Effects of habitat on settlement, growth, predation risk and survival of a temperate reef fish. Mar Ecol Prog Ser 151:225-236. doi: $10.3354 /$ meps 151225 
Vermeij M (2005) Substrate composition and adult distribution determine recruitment patterns in a Caribbean brooding coral. Mar Ecol Prog Ser 295:123-133. doi: 10.3354/meps295123

Victor BC (1983) Recruitment and population dynamics of a coral reef fish. Science 219:41920. doi: 10.1126/science.219.4583.419

Victor B (1986) Larval settlement and juvenile mortality in a recruitment-limited coral reef fish population. Ecol Monogr 56:1435-1460.

Vigliola L, Meekan MG (2002) Size at hatching and planktonic growth determine postsettlement survivorship of a coral reef fish. Oecologia 131:89-93.

Ware DM (1975) Relation Between Egg Size, Growth, and Natural Mortality of Larval Fish. J Fish Res Board Canada 32:2503-2512.

Warner R (1984) Mating behavior and hermaphroditism in coral reef fishes. Am. Sci. 72:128136

Warner RR (1987) Female choice of sites versus mates in a coral reef fish, Thalassoma bifasciatum. Anim Behav 35:1470-1478. doi: 10.1016/S0003-3472(87)80019-2

Warren EJ (1990) Spawning Patterns Within the Breeding Seasons of Favonigobius lateralis (Family: Gobiidae) and Forsterygion lapillum (Family: Tripterygiidae). MSc Thesis. University of Auckland.

Webster JP, Hoffman JI, Berdoy M (2003) Parasite infection, host resistance and mate choice: battle of the genders in a simultaneous hermaphrodite. Proc Biol Sci 270:1481-5. doi: $10.1098 / \mathrm{rspb} .2003 .2354$

Webster M (2002) Role of predators in the early post-settlement demography of coral-reef fishes. Oecologia 131:52-60. doi: 10.1007/s00442-001-0860-x

Webster M (2003) Temporal density dependence and population regulation in a marine fish. Ecology 84:623-628. doi: http://dx.doi.org/10.1890/00129658(2003)084[0623:TDDAPR]2.0.CO;2

Webster M (2004) Density dependence via intercohort competition in a coral-reef fish. Ecology 85:986-994. doi: http://dx.doi.org/10.1890/02-0576

Webster M, Hixon M (2000) Mechanisms and individual consequences of intraspecific competition in a coral-reef fish. Mar Ecol Prog Ser 196:187-194. doi: 10.3354/meps196187

Wellenreuther M (2007) Ecological factors associated with speciation in New Zealand triplefin fishes (Family Tripterygiidae ). PhD Dissertation, University of Auckland. 
Wellenreuther M, Barrett PT, Clements KD (2007a) Ecological diversification in habitat use by subtidal triplefin fishes (Tripterygiidae). Mar Ecol Prog Ser 330:235-246.

Wellenreuther M, Clements K (2007) Reproductive isolation in temperate reef fishes. Mar Biol . 152: 619-630 doi: 10.1007/s00227-007-0713-1

Wellenreuther M, Clements KD (2008) Determinants of habitat association in a sympatric clade of marine fishes. Mar Biol 154:393-402. doi: 10.1007/s00227-008-0940-0

Wellenreuther M, Syms C, Clements KD (2007b) Body size and ecological diversification in a sister species pair of triplefin fishes. Evol Ecol 22:575-592. doi: 10.1007/s10682-0079183-7

Wellenreuther M, Syms C, Clements KD (2008) Consistent spatial patterns across biogeographic gradients in temperate reef fishes. Ecography (Cop) 31:84-94.

Wellington GM (1992) Habitat selection and juvenile persistence control the distribution of two closely related Caribbean damselfishes. Oecologia 90:500-508. doi:

10.1007/BF01875443

Wells BK, Friedland KD, Clarke LM (2003) Increment patterns in otoliths and scales from mature Atlantic salmon Salmo salar. 262:293-298.

White JW, Samhouri JF, Stier AC, et al. (2010) Synthesizing mechanisms of density dependence in reef fishes: behavior, habitat configuration, and observational scale. Ecology 91:19491961. doi: 10.1890/09-0298.1

White J, Standish J, Thorrold S, Warner RR (2008) Markov chain Monte Carlo methods for assigning larvae to natal sites using natural geochemical tags. Ecol Appl 18:1901-1913.

Wilkin TA, Sheldon BC (2009) Sex differences in the persistence of natal environmental effects on life histories. Curr Biol 19:1998-2002. doi: 10.1016/j.cub.2009.09.065

Willis T (2001) Visual census methods underestimate density and diversity of cryptic reef fishes. J Fish Biol 59:1408-1411. doi: 10.1006/jfbi.2001.1721

Wilson JA (2005) Age class interactions in a marine goby, Elacatinus prochilos (Böhlke and Robins, 1968). J Exp Mar Bio Ecol 327:144-156. doi: 10.1016/j.jembe.2005.06.013

Wilson JA, Osenberg CW (2002) Experimental and observational patterns of density-dependent settlement and survival in the marine fish, Gobiosoma. Oecologia 130:205-215. doi: $10.1007 / \mathrm{s} 004420100784$ 
Zuk M, Thornhill R, Ligon J, Johnson K (1990) Parasites and mate choice in red jungle fowl. Am Zool 30:235-244.

Zymonas ND, McMahon TE (2009) Comparison of pelvic fin rays, scales and otoliths for estimating age and growth of bull trout, Salvelinus confluentus. Fish Manag Ecol 16:155164. doi: 10.1111/j.1365-2400.2008.00640.x 